\title{
A Model-Based Approach to Demodulation of Co-Channel MSK Signals
}

\author{
By \\ Yasir Ahmed \\ Thesis submitted to the faculty of the \\ Virginia Polytechnic Institute and State University \\ in partial fulfillment for the degree of \\ Master of Science \\ in \\ Electrical Engineering
}

APPROVED:

$\overline{\text { Dr. Jeffrey H. Reed, Chairman }}$

Dr. William H. Tranter, Co-Chair

Dr. R. Michael Buehrer

December 2002

Blacksburg, Virginia

Keywords: MSK Modulation, Co-Channel Interference, Minimum Variance Estimation, Autoregressive Estimation 


\title{
A Model-Based Approach to Demodulation of Co-Channel MSK Signals
}

\author{
By \\ Yasir Ahmed \\ Committee Chairman: Dr. Jeffrey H. Reed \\ Electrical Engineering
}

\begin{abstract}
Co-channel interference limits the capacity of cellular systems, reduces the throughput of wireless local area networks, and is the major hurdle in deployment of high altitude communication platforms. It is also a problem for systems operating in unlicensed bands such as the $2.4 \mathrm{GHz}$ ISM band and for narrowband systems that have been overlaid with spread spectrum systems.

In this work we have developed model-based techniques for the demodulation of cochannel MSK signals. It is shown that MSK signals can be written in the linear model form, hence a minimum variance unbiased (MVU) estimator exists that satisfies the Cramer-Rao lower bound (CRLB) with equality. This framework allows us to derive the best estimators for a single-user and a two-user case. These concepts can also be extended to wideband signals and it is shown that the MVU estimator for Direct Sequence Spread Spectrum signals is in fact a decorrelator-based multiuser detector.

However, this simple linear representation does not always exist for continuous phase modulations. Furthermore, these linear estimators require perfect channel state information and phase synchronization at the receiver, which is not always implemented in wireless communication systems. To overcome these shortcomings of the linear estimation techniques, we employed an autoregressive modeling approach. It is well known that the AR model can accurately represent peaks in the spectrum and therefore can be used as a general FM demodulator. It does not require knowledge of the exact signal model or phase synchronization at the receiver. Since it is a non-coherent reception technique, its performance is compared to that of the limiter discriminator. Simulation results have shown that model-based demodulators can give significant gains for certain phase and frequency offsets between the desired signal and an interferer.
\end{abstract}




\section{Acknowledgements}

I would like to express my gratitude to Dr. Reed and Dr. Tranter for being my advisors and for their constant encouragement and support. I would also like to thank Dr. Buehrer for serving on my advisory committee.

I would like to thank James Hicks for providing me with some excellent references and

for his insightful comments and suggestions, to Yash Vasavada for introducing me to various GMSK receiver architectures and to Pierre, Ravi, Ali and Sarfraz for reviewing my work and for the various discussions I had with them. I would also like to thank the MPRG staff for their excellent support.

This research has been sponsored by the MPRG Industrial Affiliates program, the Defense Advanced Research Project Agency (DARPA) and Raytheon through the ACN project. 


\section{Table of Contents}

I. Introduction 1

II. MSK Modulation and Co-Channel Interference 4

2.1 Introduction 4

2.2 Co-Channel Interference 4

2.3 Minimum Shift Keying 6

$\begin{array}{lll}\text { 2.3.1 Mathematical Model } & 7\end{array}$

2.3.2 Orthogonal Minimum Shift Keying 8

$\begin{array}{ll}\text { 2.3.3 OMSK Receiver Architecture } & 10\end{array}$

$\begin{array}{ll}2.3 .4 \text { Non-Orthogonal Carriers } & 12\end{array}$

2.4 Multi-Amplitude Minimum Shift Keying 14

2.5 Simulation Results 16

2.6 Summary

III. Minimum Variance Unbiased Estimation 21

$\begin{array}{ll}3.1 \text { Introduction } & 21\end{array}$

3.2 Estimation Problem 21

3.2.1 Minimum Variance Unbiased Estimation 22

$\begin{array}{ll}\text { 3.2.2 Cramer-Rao Lower Bound } & 23\end{array}$

3.2.3 Linear Models 24

3.3 Minimum Variance Unbiased Estimator for Narrowband Signals 26

3.4 Minimum Variance Unbiased Estimator for Wideband Signals 33

$\begin{array}{ll}3.5 & \text { The Decorrelator } \\ 3.6 & 35\end{array}$

3.6 Geometrical Interpretation of the Decorrelator 37

$\begin{array}{lll}3.7 & \text { Simulation Results } & 40\end{array}$

3.8 Summary 43

IV. Autoregressive Parameter Estimation $\quad 45$

4.1 Introduction 45

4.2 Instantaneous Frequency 46

4.3 Frequency Estimation Techniques $\quad 47$

4.3.1 Classical Techniques 48

4.3.2 Rational Transfer Function Modeling $\quad 50$

4.3.3 Subspace Methods $\quad 53$

4.4 Model Selection 58

4.5 Autoregressive Parameter Estimation $\quad 59$

4.5.1 Maximum Likelihood Estimation $\quad 60$

4.5.2 Least Squares Approach 63

4.5.3 Sequential Approach $\quad 66$

4.6 Frequency Component Determination 67

4.7 Limiter Discriminator as a Linear Predictor 68

4.8 Simulation Results $\quad 69$

$\begin{array}{lll}4.9 & \text { Summary } & 77\end{array}$ 
V. Conclusions and Future Work 79

$\begin{array}{lll}5.1 \text { Conclusions } & 79\end{array}$

5.2 Future Work 81

$\begin{array}{ll}\text { References } & 82\end{array}$

Appendix A $\quad 85$ 


\section{List of figures}

2.1 Parallel MSK receiver 7

2.2 In-phase and quadrature components of MSK signal 8

2.3 Signal constellation of the MSK signals for (a) odd (b) even sampling 9

2.4 Phase trellis for the two MSK signals. One possible path for each of the 10 two signals is high lightened

2.5 Receiver architecture of MSK 10

2.6 Signal constellation for MAMSK (a) constituent signals have equal 15

2.7 Power spectra of MSK, OMSK/MAMSK and QPSK 15

2.8 Error performance of MSK signal in an AWGN channel with and 16

2.9 Error performance when the interference is not orthogonal $(\mathrm{SIR}=0 \mathrm{~dB}) \quad 17$

2.10 Error performance for various SIRs and phase deviations, $\mathrm{Eb} / \mathrm{No}=\quad 18$ $10 \mathrm{~dB}$

2.11 Error performance for various SIRs and phase deviations, Eb/No $=\quad 18$ $18 \mathrm{~dB}$

2.12 Error performance for different values of frequency separation 19

2.13 (a) Both tones overlap (b) One tone overlaps 19

$\begin{array}{lll}3.1 & \text { Correlation receiver } & 28\end{array}$

3.2 Signal constellation of a QPSK signal (a) ideal (b) skewed 29

3.3 Comparison of MF and MVU for non-orthogonal carriers. Phase error $=30$ 10 degrees

3.4 Comparison of MF and MVU for non-orthogonal carriers. Phase error $=\quad 30$ 20 degrees

3.5 Checkerboard plots for various phase error values. Lighter shades 32

3.6 Decorrelating detector for the synchronous channel 34

3.7 Geometrical representation of matched filter for two users 38

3.8 Geometrical representation of a decorrelator for two users 39

3.9 MSK bit error rate at $\mathrm{SIR}=0 \mathrm{~dB}$ and $\mathrm{Eb} / \mathrm{No}=18 \mathrm{~dB} \quad 40$

3.10 MSK bit error rate at $\mathrm{SIR}=0 \mathrm{~dB}$ and phase deviation $=10 \mathrm{deg} \quad 41$

$3.11 \quad \mathrm{MSK}$ bit error rate at $\mathrm{Eb} / \mathrm{No}=18 \mathrm{~dB}$ and $\mathrm{SIR}=0,1,2,3 \mathrm{~dB}$

$3.12 \quad$ MSK bit error rate at $\mathrm{SIR}=0 \mathrm{~dB}$ and $\mathrm{Eb} / \mathrm{No}=12 \mathrm{~dB} \quad 42$

3.13 MSK bit error rate for $\mathrm{SIR}=0 \mathrm{~dB}$, frequency offset $=0.5$ and phase $\quad 43$

4.1 Steps involved in Model-based spectral estimation 58

4.2 Signal flow graph for the AR model 59

4.3 Cramer-Rao lower bound for the AR(1) PSD 63

4.4 Pole-zero plot of a fifth order filter for noise and high noise case 69

4.5 Performance of LD and model-based demodulator for various filter 72

lengths

4.6 Performance of model-based demodulator for various values of the 72 
phase difference between the signal of interest and an interferer

4.7 Performance of model-based demodulator with LMS update

4.8 Performance of model-based demodulator using angle of filter coefficients

4.9 Comparison of various model-based demodulation schemes 74

4.10 Performance of limiter discriminator for various carrier frequency 75 separations

4.11 Performance of model-based demodulator for various carrier frequency $\quad 75$ separations

4.12 Comparison of limiter discriminator and model-based demodulator for $\quad 76$ carrier frequency separation of $1 / 2 \mathrm{~T}$

4.13 Comparison of model-based demodulator and limiter discriminator in $\quad 76$ Rayleigh fading environment $(\mathrm{FdT}=0.002, \mathrm{~Eb} / \mathrm{No}=20 \mathrm{~dB})$ 


\section{Chapter I Introduction}

As the demand for high data rate wireless services continues to increase, efficient utilization of available spectrum becomes extremely important. Furthermore the number of users requiring these services also continues to grow. The capacity of typical cellular systems can be increased by either reducing the cell size or cluster size. Reducing the cell size is a costly option, as it requires a higher number of base stations in a given geographical area. Reducing the cluster size does not increase the number of base stations however it reduces the distance between cells employing the same frequency band and this results in a higher amount of interference. These cells are called co-channel cells and the interference is appropriately called co-channel interference. The number of simultaneous co-channel interferers can be reduced by dividing each cell into sectors and using only a portion of the frequency band in each sector. However this would require installation of directional antennas at all the base stations.

The problem of co-channel interference is prevalent in most wireless communication systems. These include systems operating in unlicensed bands such as the $2.4 \mathrm{GHz}$ ISM band, systems employing an airborne communications node and narrowband systems that have been overlaid with spread spectrum systems. This is also a problem for $3 \mathrm{G}$ systems where some narrowband systems continue to operate in the spectrum allocated for $3 \mathrm{G}$ [Por01] and for military systems that share the VHF band with commercial systems. Based upon the nature of interference, interference rejection techniques can be broadly categorized into spread spectrum techniques and non-spread spectrum techniques [Las97].

The goal of our research is to study the problem of co-channel interference for narrowband signals and to develop interference rejection techniques that can be implemented without modifying the existing system. It is assumed that we do not have 
antenna arrays available either at the base station or the mobile. It is further assumed that there is a small offset in the carrier frequencies of the co-channel signals. This is a reasonable assumption as there is always some inaccuracy in the carrier generation circuitry at the transmitter. The modulation scheme selected for this work is Gaussian Minimum Shift Keying (GMSK) as it is one of the most popular modulation schemes being used today.

In chapter 2 we define the problem of co-channel interference in more detail and present the mathematical model for MSK signals. We then consider a special case of co-channel interference when the two MSK signals are orthogonal to each other and propose a receiver architecture similar to the typical correlation receiver. We next consider the more general case when the two MSK signals are not orthogonal. The case of orthogonal MSK signals is then compared to Multi-Amplitude MSK. Finally the performance of the correlation receiver is evaluated for different test cases.

The correlation receiver demodulates each signal separately while treating the others as noise. It is obvious that this is not the optimum solution as no information about the interfering signals is used in demodulating the signal of interest. This leads us to the idea of jointly detecting the signal of interest and the interferer. The minimum variance unbiased (MVU) estimator is presented as a method of jointly detecting both the signals. It is also shown that the MVU estimator is essentially a decorrelator detector that has been extensively studied for CDMA signals.

In chapters 2 and 3 we have assumed that the signal can be represented by a linear model and have thus been able to use linear estimation techniques. We have also assumed that we have perfect phase synchronization at the receiver. However in practice this is usually not the case. It is difficult to represent GMSK signals in linear model form and the phase synchronization circuitry is some times not implemented at the receiver due to economic constraints. We therefore explore frequency estimation techniques for demodulation of GMSK signals. These techniques are essentially non-coherent demodulation schemes that require no phase synchronization. Furthermore there might be some advantage in using 
high-resolution frequency estimation techniques when the carrier frequencies of the two co-channel signals are not the same.

Frequency estimation techniques can be divided into 3 categories, namely

1. Classical techniques based on the Fourier transform

2. Rational transfer function models

3. Subspace methods

We have selected an autoregressive modeling approach that falls into the second category. This is because Classical techniques have limited frequency resolution and subspace methods are only applicable to signals having discrete frequency components. Furthermore the AR modeling approach has a close resemblance with differential demodulation and has been successfully used for demodulation of co-channel AMPS signals [We196]. The performance of the model-based demodulator is compared with the limiter discriminator, as it is the most popular non-coherent demodulation technique for GMSK type signals. Finally conclusions and suggestions for future work are presented in chapter 5 . 


\section{Chapter II MSK Modulation and Co-Channel Interference}

\subsection{Introduction}

Co-channel interference limits the capacity of cellular systems [Las97], reduces the throughput of wireless local area networks (WLANs) and personal area networks (WPANs) [Amr01], and is also the major hurdle in deployment of high altitude communication platforms [Saf00]. In this chapter we will study the effect of co-channel interference on Minimum Shift Keyed (MSK) signals, as it is the most popular modulation format. GMSK, Gaussian pulse shaped MSK, has been adopted by many cellular standards including GSM (Global System for Mobile Communications), CDPD (Cellular Digital Packet Data) and DECT (Digital European Cordless Telephone). Bluetooth also uses a variant of GMSK called GFSK with a frequency separation less than the theoretical minimum required for orthogonal detection of two tones.

We will briefly describe the problem of co-channel interference followed by an introduction to the MSK signal and its mathematical model. We will then consider a special case of co-channel interference when the two signals are orthogonal to each other. We next quantify the interference when the signals are not orthogonal. Finally simulation results are presented for both the cases.

\subsection{Co-Channel Interference}

Co-channel interference is a major problem in current as well as emerging wireless communication systems, e.g., in cellular radio, Bluetooth and wireless local area networks and systems employing an airborne communications node. In cellular radio networks the coverage area is split into cells and the same set of frequencies is used for cells that are separated geographically. These are called co-channel cells. The minimum 
number of cells that occupy the available frequency band is called the cluster size and is usually equal to 4, 7 or 12 [Rap96]. A smaller cluster size allows the same frequencies to be used more often and this results in a higher system capacity. However, this also results in reduction in separation between co-channel cells and a higher amount of co-channel interference. Therefore there is a tradeoff between increasing the system capacity and reducing co-channel interference. One solution to this problem is to divide each cell into sectors by using directional antennas thereby reducing the number of simultaneous cochannel interferers. However this is a costly solution, as it requires installation of new equipment at all the base stations.

Bluetooth is a short range wireless communication standard designed for the $2.4-\mathrm{GHz}$ ISM band. It uses frequency hopping spread spectrum with GFSK modulation and provides data rates of up to $1 \mathrm{Mbps}$ [Amr01]. A Bluetooth network called a piconet consists of a master communicating with several slaves in a time-division duplex fashion. Since it operates in the $2.4-\mathrm{GHz}$ unlicensed band a piconet is prone to interference from several other devices operating in the same band as well as other piconets that are in proximity. The major interferers are IEEE $802.11 \mathrm{~b}$ wireless LAN devices and microwave ovens. The impact of interference on Bluetooth and IEEE 802.11b systems has been studied extensively and it has been shown that it can cause severe degradation in performance [Go101], [Fai01] especially in dense office environments.

An airborne communications node $(\mathrm{ACN})$ is a communications platform hovering above the surface of the earth that provides extended coverage in remote regions [Saf00]. It also reduces the number of handoffs required for highly mobile users. The ACN can either be a simple repeater that just retransmits the signal after amplification or it can be a complete basestation providing coverage in a large geographical area. Since the ACN is positioned several miles above the earth's surface it is susceptible to interference from a large number of sources. Furthermore, these sources have a Line of Sight (LOS) with the $\mathrm{ACN}$ and thus the interference is quite significant [Saf00]. Although these signals overlap in time and frequency they can be separated spatially by using antenna arrays. However it is difficult to mount large antenna arrays on airborne platforms due to the limitations on 
size and weight. The antenna arrays also have a detrimental effect on the aerodynamics of the platform. Therefore it is desirable to come up with signal processing algorithms that can separate co-channel interferers using a single receive antenna.

Co-channel interference rejection techniques can be broadly divided into two categories depending upon whether the interfering signal is treated as noise or as another signal that needs to be jointly detected. The techniques that fall into the first category perform adequately when there is sufficient difference in power levels of the signal of interest (SOI) and the signal not of interest (SNOI). However they fail if the two signals have near equal power. The most powerful narrowband joint detection techniques are based on joint maximum likelihood sequence estimation (JMLSE). [Ran95] formulates the problem of joint detection of $N$ co-channel signals in a channel of length $L$ and shows that significant gains can be obtained over a conventional receiver provided that the power of non-dominant interferers (that are neglected) is much smaller. The number of states in the JMLSE trellis is equal $2^{N L / 2}$ which becomes prohibitively complex as the number of signals or the channel length increases. [Mph96] extends this work to GMSK signals and shows that the performance of JMLSE receiver is highly dependent upon the phase difference between the two signals however he does not give an explanation for this behavior.

\subsection{Minimum Shift Keying}

Minimum Shift Keying (MSK) is a spectrally efficient modulation scheme that is being widely used in many wireless communication systems. It is basically a type of Continuous Phase Frequency Shift Keying (CPFSK) with modulation index, h, equal to 0.5. It has a constant envelope and can be demodulated coherently or non-coherently. When coherently demodulated the performance of MSK is the same as BPSK, QPSK and OQPSK[Pas79]. MSK can be demodulated coherently using a parallel receiver architecture shown in Fig. 2.1. This is similar to a QPSK receiver except that output of the correlators is sampled with an offset T. Also note that the bit decisions in each arm are made over 2T. A detailed discussion about the properties of MSK and its transmitter 
and receiver architectures can be found in [Pas79] and [Sun86]. Murota [Mur81] analyzes the spectral properties of Gaussian filtered MSK (GMSK), its error performance and proposes transmitter and receiver architectures.

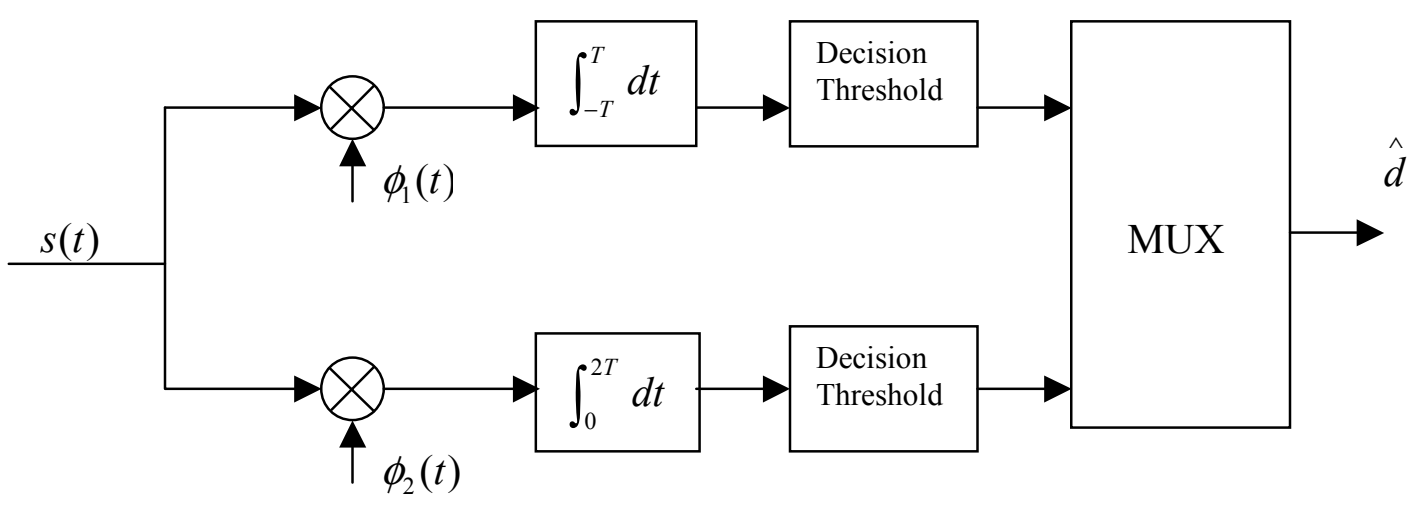

Fig. 2.1 Parallel MSK receiver

\subsubsection{Mathematical Model}

The MSK signal can be expressed as

$$
s_{1}(t)=a_{1} \sqrt{\frac{2 E_{b}}{T_{b}}} \cos \left(\frac{\pi}{2 T_{b}} t\right) \cos \left(2 \pi f_{c} t\right)+b_{1} \sqrt{\frac{2 E_{b}}{T_{b}}} \sin \left(\frac{\pi}{2 T_{b}} t\right) \sin \left(2 \pi f_{c} t\right)
$$

where $E_{b}$ is the energy per bit, $T_{b}$ is the bit duration, $f_{c}$ is the carrier frequency and $a_{1}, b_{1}= \pm 1$ are the information bits. The basis functions for the MSK signal can be defined over $2 T_{b}$ as

$$
\begin{gathered}
\phi_{1}(t)=\sqrt{\frac{2}{T_{b}}} \cos \left(\frac{\pi}{2 T_{b}} t\right) \cos \left(2 \pi f_{c} t\right) \\
\phi_{2}(t)=\sqrt{\frac{2}{T_{b}}} \sin \left(\frac{\pi}{2 T_{b}} t\right) \sin \left(2 \pi f_{c} t\right)
\end{gathered}
$$

The in-phase and quadrature components of the MSK signal are shown in Fig. 2.2. It can 


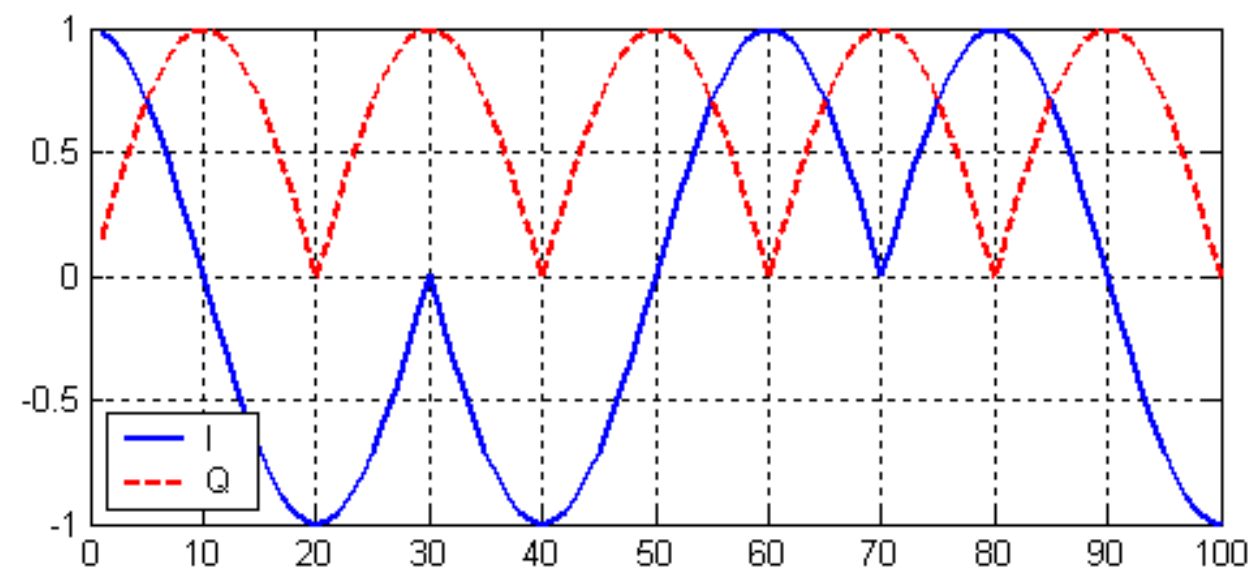

Fig. 2.2 In-phase and quadrature components of MSK signal (10 samples per symbol)

be seen that at each sampling instant (10n) only the in-phase or quadrature component carries information while the other is zero. This leads us to the idea that if another MSK signal is combined with this signal such that its I and Q components are orthogonal to the I and Q components of the original MSK signal then we can successfully demodulate both of them.

\subsubsection{Orthogonal Minimum Shift Keying}

This new MSK signal that is orthogonal to the original MSK signal can be defined as

$$
s_{2}(t)=a_{2} \sqrt{\frac{2 E_{b}}{T_{b}}} \sin \left(\frac{\pi}{2 T_{b}} t\right) \cos \left(2 \pi f_{c} t\right)+b_{2} \sqrt{\frac{2 E_{b}}{T_{b}}} \cos \left(\frac{\pi}{2 T_{b}} t\right) \sin \left(2 \pi f_{c} t\right)
$$

and the basis functions for this new signal are defined over $2 T_{b}$ as

$$
\begin{aligned}
& \phi_{3}(t)=\sqrt{\frac{2}{T_{b}}} \cos \left(\frac{\pi}{2 T_{b}} t\right) \sin \left(2 \pi f_{c} t\right) \\
& \phi_{4}(t)=\sqrt{\frac{2}{T_{b}}} \sin \left(\frac{\pi}{2 T_{b}} t\right) \cos \left(2 \pi f_{c} t\right)
\end{aligned}
$$


It can be shown that the basis functions for this signal are orthonormal to the original basis functions. The signal constellation of both the signals is shown in Fig. 2.3. It is seen that at each sampling instant the I and Q components carry information from only one of the two MSK signals. The phase trellis of the two signals is shown in Fig. 2.4.

Now we define a new MSK signal called Orthogonal MSK (OMSK) that is the sum of the two signals defined above.

$$
s(t)=s_{1}(t)+s_{2}(t)
$$

or

$s(t)=\sqrt{\frac{2 E_{b}}{T_{b}}}\left[a_{1} \cos \left(\frac{\pi}{2 T_{b}} t\right)+a_{2} \sin \left(\frac{\pi}{2 T_{b}} t\right)\right] \cos \left(2 \pi f_{c} t\right)+\sqrt{\frac{2 E_{b}}{T_{b}}}\left[b_{1} \sin \left(\frac{\pi}{2 T_{b}} t\right)+b_{2} \cos \left(\frac{\pi}{2 T_{b}} t\right)\right] \sin \left(2 \pi f_{c} t\right)$

It should be noted that in this form the OMSK signal resembles the QPSK signal.

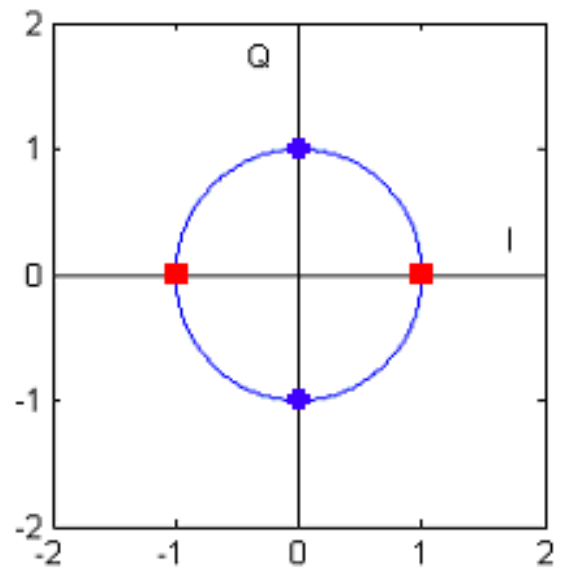

(a)

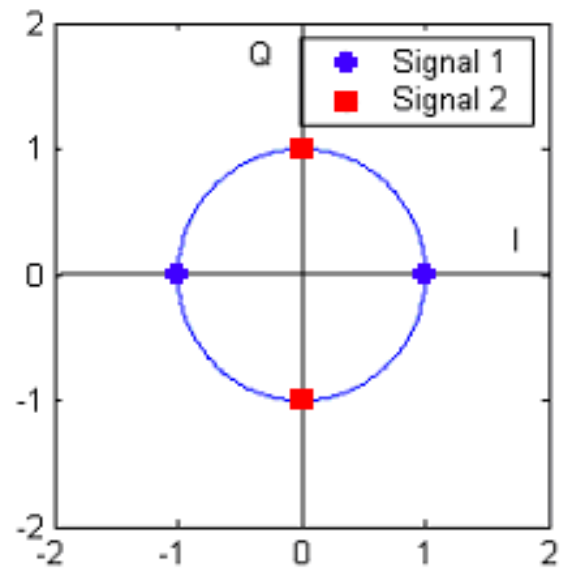

(b)

Fig. 2.3 Signal constellation of the MSK signals for (a) odd and (b) even sampling instants 


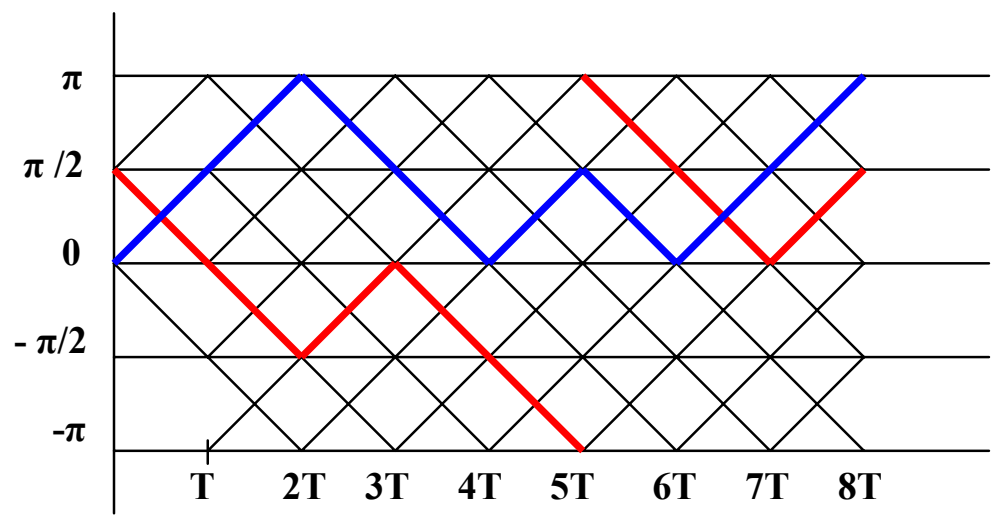

Fig. 2.4. Phase trellis (modulo $2 \pi$ ) for the two MSK signals. One possible path for each of the two signals is high lightened.

\subsubsection{OMSK Receiver Architecture}

The OMSK signal can be demodulated using a receiver architecture similar to the parallel receiver architecture for MSK. This is shown in Fig. 2.5.

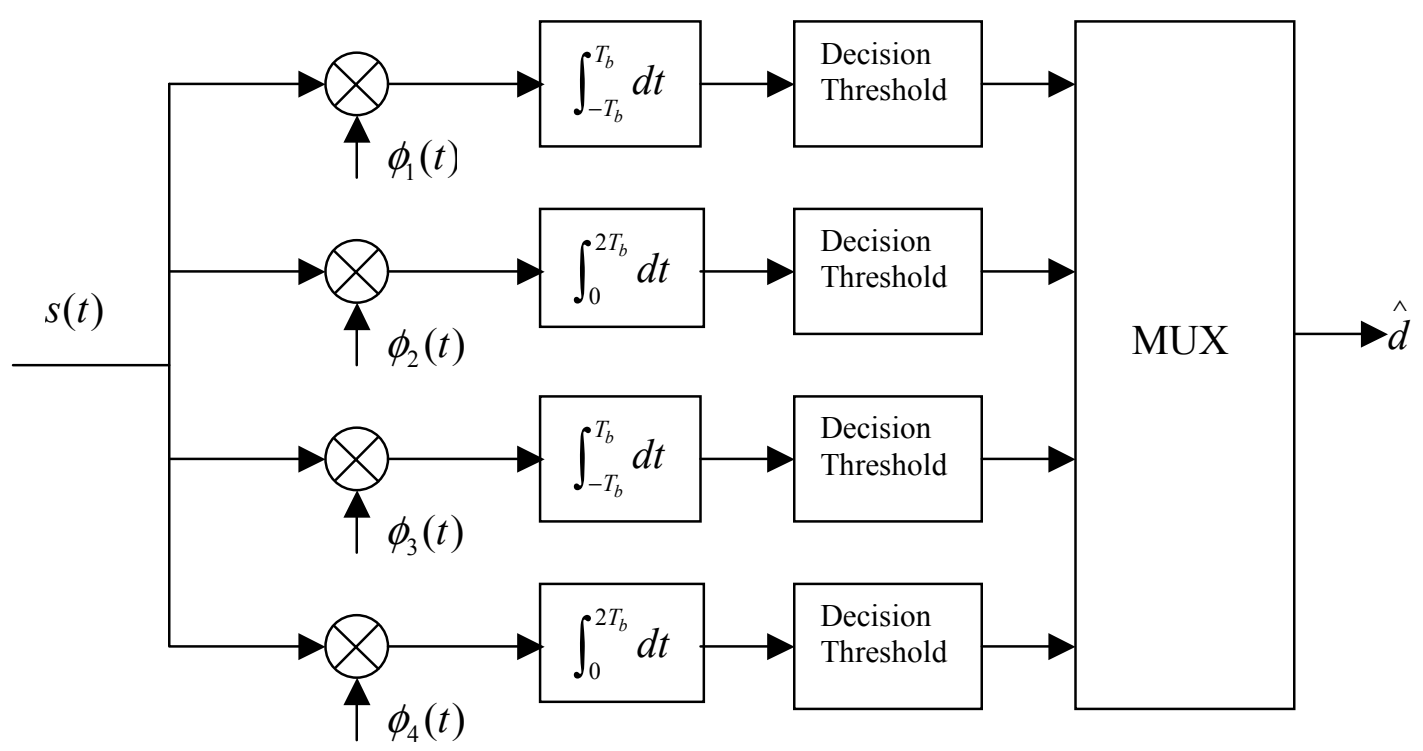

Fig. 2.5 Receiver architecture for OMSK

The only difference between this receiver and the MSK receiver is that we have four correlators instead of two. Now let us verify the correctness of this receiver architecture 
by calculating the output of one of the correlators. From (2.2) and (2.8) $s(t) \phi_{1}(t)$ can be written as

$$
\begin{aligned}
& \sqrt{\frac{2 E_{b}}{T_{b}}}\left[\left\{a_{1} \cos \left(\frac{\pi t}{2 T_{b}}\right)+a_{2} \sin \left(\frac{\pi t}{2 T_{b}}\right)\right\} \cos \left(2 \pi f_{c} t\right)+\left\{b_{1} \sin \left(\frac{\pi t}{2 T_{b}}\right)+b_{2} \cos \left(\frac{\pi t}{2 T_{b}}\right)\right\} \sin \left(2 \pi f_{c} t\right)\right] . \\
& {\left[\sqrt{\frac{2}{T_{b}}} \cos \left(\frac{\pi t}{2 T_{b}}\right) \cos \left(2 \pi f_{c} t\right)\right]}
\end{aligned}
$$$$
=\frac{2 \sqrt{E_{b}}}{T_{b}}\left[a_{1} \cos \left(\frac{\pi t}{2 T_{b}}\right) \cos \left(2 \pi f_{c} t\right)+a_{2} \sin \left(\frac{\pi t}{2 T_{b}}\right) \cos \left(2 \pi f_{c} t\right)+b_{1} \sin \left(\frac{\pi t}{2 T_{b}}\right) \sin \left(2 \pi f_{c} t\right)+b_{2} \cos \left(\frac{\pi t}{2 T_{b}}\right) \sin \left(2 \pi f_{c} t\right)\right] .
$$$$
\left[\cos \left(\frac{\pi t}{2 T_{b}}\right) \cos \left(2 \pi f_{c} t\right)\right]
$$

$$
\left.\begin{array}{rl}
=\frac{2 \sqrt{E_{b}}}{T_{b}}\left[\begin{array}{l}
a_{1} \cos ^{2}\left(\frac{\pi t}{2 T_{b}}\right) \cos ^{2}\left(2 \pi f_{c} t\right)+a_{2} \sin \left(\frac{\pi t}{2 T_{b}}\right) \cos \left(\frac{\pi t}{2 T_{b}}\right) \cos ^{2}\left(2 \pi f_{c} t\right)+ \\
b_{1} \sin \left(\frac{\pi t}{2 T_{b}}\right) \cos \left(\frac{\pi t}{2 T_{b}}\right) \sin \left(2 \pi f_{c} t\right) \cos \left(2 \pi f_{c} t\right)+b_{2} \cos ^{2}\left(\frac{\pi t}{2 T_{b}}\right) \sin \left(2 \pi f_{c} t\right) \cos \left(2 \pi f_{c} t\right)
\end{array}\right] \\
\left.\left.=\frac{2 \sqrt{E_{b}}}{T_{b}}\left[\begin{array}{l}
\frac{a_{1}}{4}\left(1+\cos \left(\frac{\pi t}{T_{b}}\right)\right)\left(1+\cos \left(4 \pi f_{c} t\right)+\frac{a_{2}}{4}\left(\sin \left(\frac{\pi t}{T_{b}}\right)\right)\left(1+\cos \left(4 \pi f_{c} t\right)+\right]\right. \\
\frac{b_{1}}{4} \sin \left(\frac{\pi t}{T_{b}}\right) \sin \left(4 \pi f_{c} t\right)+\frac{b_{2}}{4}\left(1+\cos \left(\frac{\pi t}{T_{b}}\right)\right) \sin \left(4 \pi f_{c} t\right)
\end{array}\right]+\frac{\pi t}{T_{b}}\right)\right\}+ \\
=\frac{2 \sqrt{E_{b}}}{T_{b}}
\end{array}\right] \begin{aligned}
& \frac{a_{2}}{4}\left\{\begin{array}{l}
1+\cos \left(\frac{\pi t}{T_{b}}\right)+\cos \left(4 \pi f_{c} t\right)+\frac{1}{2} \cos \left(4 \pi f_{c} t+\frac{\pi t}{T_{b}}\right)+\frac{1}{2} \cos \left(4 \pi f_{c} t\right. \\
\left.\sin \left(\frac{\pi t}{T_{b}}\right)+\frac{1}{2} \sin \left(4 \pi f_{c} t+\frac{\pi t}{T_{b}}\right)-\frac{1}{2} \sin \left(4 \pi f_{c} t-\frac{\pi t}{T_{b}}\right)\right\}+ \\
\frac{b_{1}}{4}\left\{\frac{1}{2} \cos \left(4 \pi f_{c} t-\frac{\pi t}{T_{b}}\right)-\frac{1}{2} \cos \left(4 \pi f_{c} t+\frac{\pi t}{T_{b}}\right)\right\}+ \\
\frac{b_{2}}{4}\left\{\begin{array}{l}
\left.\sin \left(4 \pi f_{c} t\right)+\frac{1}{2} \sin \left(4 \pi f_{c} t+\frac{\pi t}{T_{b}}\right)+\frac{1}{2} \sin \left(4 \pi f_{c} t-\frac{\pi t}{T_{b}}\right)\right\}
\end{array}\right]
\end{array}\right]
\end{aligned}
$$

Taking the integral of $s(t) \phi_{1}(t)$ over the appropriate interval. 


$$
\begin{aligned}
& \int_{-T_{b}}^{T_{b}} s(t) \phi_{1}(t) d t \\
& =\frac{2 \sqrt{E_{b}}}{T_{b}} \int_{-T_{b}}^{T_{b}}\left[\frac{a_{1}}{4}\left(1+\cos \frac{\pi t}{T_{b}}\right)+\frac{a_{2}}{4} \sin \left(\frac{\pi t}{T_{b}}\right)\right] d t \\
& =\frac{2 \sqrt{E_{b}}}{T_{b}}\left[\frac{a_{1} t}{4}+\frac{a_{1} T_{b}}{4 \pi} \sin \left(\frac{\pi t}{T_{b}}\right)-\frac{a_{2} T_{b}}{4 \pi} \cos \left(\frac{\pi t}{T_{b}}\right)\right]_{-T_{b}}^{T_{b}} \\
& =\frac{2 \sqrt{E_{b}}}{T_{b}}\left[\frac{a_{1}}{4}\left(2 T_{b}\right)\right] \\
& =a_{1} \sqrt{E_{b}}
\end{aligned}
$$

It can also be shown that

$$
\begin{aligned}
& \int_{0}^{2 T_{b}} s(t) \phi_{2}(t) d t=b_{1} \sqrt{E_{b}} \\
& \int_{-T_{b}}^{T_{b}} s(t) \phi_{3}(t) d t=b_{2} \sqrt{E_{b}} \\
& \int_{0}^{2 T_{b}} s(t) \phi_{4}(t) d t=a_{2} \sqrt{E_{b}}
\end{aligned}
$$

These results are for the case when there is no noise. However, for the case of an AWGN channel the received signal, $r(t)$, would also have a noise component.

$$
r(t)=s(t)+n(t)
$$

Therefore the output of the correlator will also have a noise term that can be defined as

$$
n_{k}(t)=\int_{2 T} \phi_{k}(t) n(t) d t
$$

It must be noted that although the two MSK signals are orthogonal to each other the error performance is still worse than MSK/BPSK. The reason for this behavior is not very obvious at the moment. 


\subsubsection{Non-Orthogonal Carriers}

So far we have considered the case when the two MSK signals are orthogonal to each other. Let us now quantify the amount of interference between the two MSK signals if they are not completely orthogonal. We introduce a carrier phase error $\theta$ in the equation for the OMSK signal.

$$
\begin{aligned}
s(t) & =\sqrt{\frac{2 E_{b}}{T_{b}}} \cos \left(\frac{\pi t}{2 T_{b}}\right)\left[a_{1} \cos \left(2 \pi f_{c} t\right)+b_{2} \sin \left(2 \pi f_{c} t+\theta\right)\right] \\
& +\sqrt{\frac{2 E_{b}}{T_{b}}} \sin \left(\frac{\pi t}{2 T_{b}}\right)\left[a_{2} \cos \left(2 \pi f_{c} t+\theta\right)+b_{1} \sin \left(2 \pi f_{c} t\right)\right]
\end{aligned}
$$

Now let us again calculate the output of the one of the correlators.

$$
s(t) \phi_{1}(t)
$$

$$
\begin{aligned}
& =\sqrt{\frac{2 E_{b}}{T_{b}}} \cos \left(\frac{\pi t}{2 T_{b}}\right)\left[a_{1} \cos \left(2 \pi f_{c} t\right)+b_{2} \sin \left(2 \pi f_{c} t+\theta\right)\right] \\
& +\sqrt{\frac{2 E_{b}}{T_{b}}} \sin \left(\frac{\pi t}{2 T_{b}}\right)\left[a_{2} \cos \left(2 \pi f_{c} t+\theta\right)+b_{1} \sin \left(2 \pi f_{c} t\right)\right] \cdot\left[\sqrt{\frac{2}{T_{b}}} \cos \left(\frac{\pi t}{2 T_{b}}\right) \cos \left(2 \pi f_{c} t\right)\right]
\end{aligned}
$$

The second term of (2.11) is obviously orthogonal to the basis function $\phi_{1}(t)$. Therefore

$$
s(t) \phi_{1}(t)
$$

$=\sqrt{\frac{2 E_{b}}{T_{b}}} \cos \left(\frac{\pi t}{2 T_{b}}\right)\left[a_{1} \cos \left(2 \pi f_{c} t\right)+b_{2} \sin \left(2 \pi f_{c} t+\theta\right)\right]$.

$\left[\sqrt{\frac{2}{T_{b}}} \cos \left(\frac{\pi t}{2 T_{b}}\right) \cos \left(2 \pi f_{c} t\right)\right]$

$=\frac{2 \sqrt{E_{b}}}{T_{b}} \cos ^{2}\left(\frac{\pi t}{2 T_{b}}\right)\left[a_{1} \cos ^{2}\left(2 \pi f_{c} t\right)+b_{2} \sin \left(2 \pi f_{c} t+\theta\right) \cos \left(2 \pi f_{c} t\right)\right]$

$=\frac{\sqrt{E_{b}}}{T_{b}}\left(1+\cos \left(\frac{\pi t}{T_{b}}\right)\right)\left[\frac{a_{1}}{2}\left(1+\cos \left(4 \pi f_{c} t\right)\right)+\frac{b_{2}}{2} \sin \left(4 \pi f_{c} t+\theta\right)+\frac{b_{2}}{2} \sin (\theta)\right]$ 


$$
\begin{aligned}
& =\frac{\sqrt{E_{b}}}{T_{b}}\left[\frac{a_{1}}{2}+\frac{a_{1}}{2} \cos \left(4 \pi f_{c} t\right)+\frac{b_{2}}{2} \sin \left(4 \pi f_{c} t+\theta\right)+\frac{b_{2}}{2} \sin (\theta)\right] . \\
& \frac{\sqrt{E_{b}}}{T_{b}}\left[\begin{array}{l}
\frac{a_{1}}{2} \cos \left(\frac{\pi t}{T_{b}}\right)+\frac{a_{1}}{4} \cos \left(4 \pi f_{c} t+\frac{\pi t}{T_{b}}\right)+\frac{a_{1}}{4} \cos \left(4 \pi f_{c} t-\frac{\pi t}{T_{b}}\right) \\
+\frac{b_{2}}{4} \sin \left(4 \pi f_{c} t+\theta+\frac{\pi t}{T_{b}}\right)+\frac{b_{2}}{4} \sin \left(4 \pi f_{c} t+\theta-\frac{\pi t}{T_{b}}\right)+\frac{b_{2}}{4} \sin \left(\theta+\frac{\pi t}{T_{b}}\right)+\frac{b_{2}}{4} \sin \left(\theta-\frac{\pi t}{T_{b}}\right)
\end{array}\right]
\end{aligned}
$$

Taking the integral of $s(t) \phi_{1}(t)$ over the appropriate interval.

$$
\begin{aligned}
& \int_{-T_{b}}^{T_{b}} s(t) \phi_{1}(t) d t \\
& =\frac{\sqrt{E_{b}}}{T_{b}} \int_{-T_{b}}^{T_{b}}\left[\frac{a_{1}}{2}+\frac{b_{2}}{2} \sin \theta\right] d t+\frac{\sqrt{E_{b}}}{T_{b}} \int_{-T_{b}}^{T_{b}}\left[\frac{a_{1}}{2} \cos \left(\frac{\pi t}{2 T_{b}}\right)+\frac{b_{2}}{4} \sin \left(\theta+\frac{\pi t}{T_{b}}\right)+\frac{b_{2}}{4} \sin \left(\theta-\frac{\pi t}{T_{b}}\right)\right] d t \\
& =\sqrt{E_{b}} a_{1}+\sqrt{E_{b}} b_{2} \sin \theta
\end{aligned}
$$

As expected the amount of interference is proportional to the phase error $\theta$ (modulo 90 degrees) and is maximum for a phase error of 90 degrees.

\subsection{Multi-Amplitude Minimum Shift Keying}

The multi-level MSK signal described in section 2.3.2 can be considered a special case of multi-amplitude minimum shift keying (MAMSK) [Web78]. MAMSK is a bandwidth efficient modulation scheme that has the continuous phase property of MSK and provides higher spectral efficiency by using multilevel modulation. However, for MAMSK there is no requirement for the two signals to be orthogonal, in fact, they are co-phased. Furthermore, the amplitudes of the constituent signals are not equal and this prevents the phase trajectory from passing through the origin (Fig. 2.6). For example, a two level MAMSK signal can be expressed as

$$
s(t)=2 A \cos \left[2 \pi f_{c} t+\phi_{2}(t ; I)\right]+A \cos \left[2 \pi f_{c} t+\phi_{1}(t ; J)\right]
$$

where 


$$
\begin{array}{ll}
\phi_{2}(t ; I)=\frac{\pi}{2} \sum_{k=-\infty}^{n-1} I_{k}+\frac{\pi}{2 T_{s}} I_{n}(t-n T) & n T \leq t \leq(n+1) T \\
\phi_{1}(t ; J)=\frac{\pi}{2} \sum_{k=-\infty}^{n-1} J_{k}+\frac{\pi}{2 T_{s}} J_{n}(t-n T) & n T \leq t \leq(n+1) T
\end{array}
$$

where $I_{k}$ and $J_{k}$ are a function of the information sequence [Pro01].

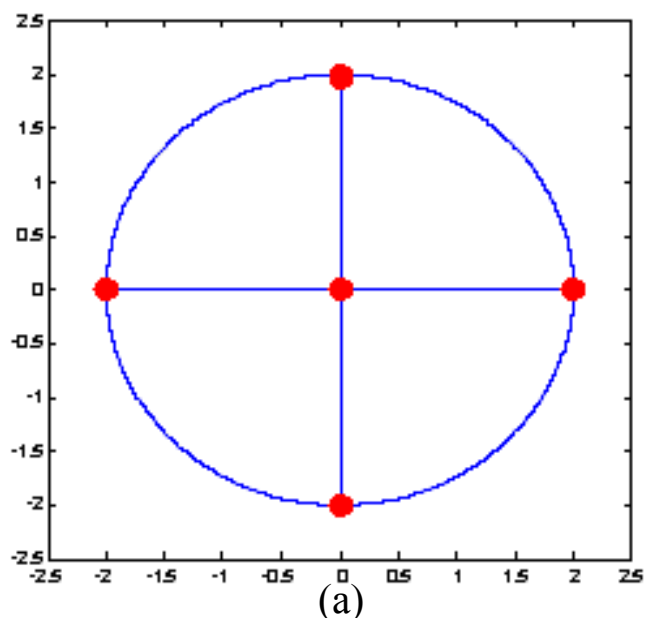

(a)

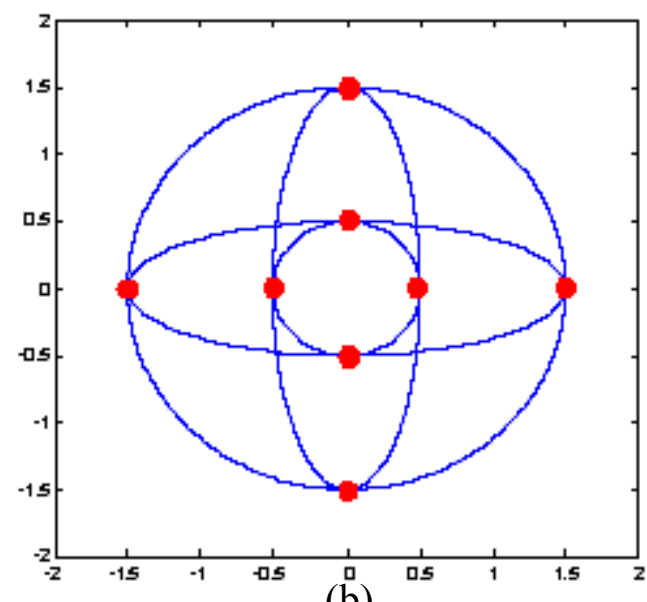

(b)

Fig. 2.6. Signal constellation for MAMSK (a) Constituent signals have equal amplitude (b) Constituent signals have unequal amplitude

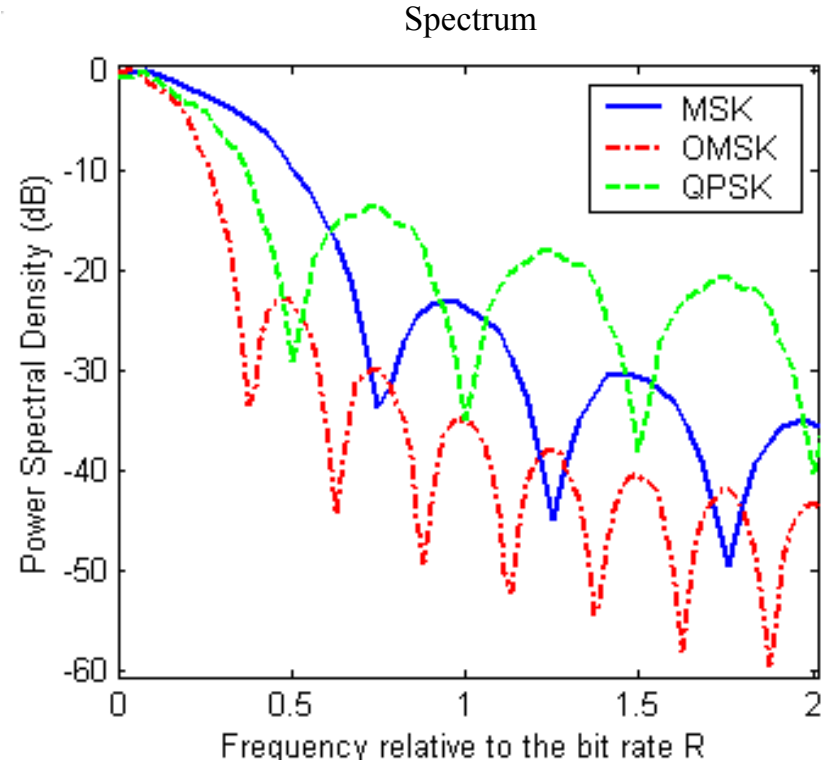

(a)

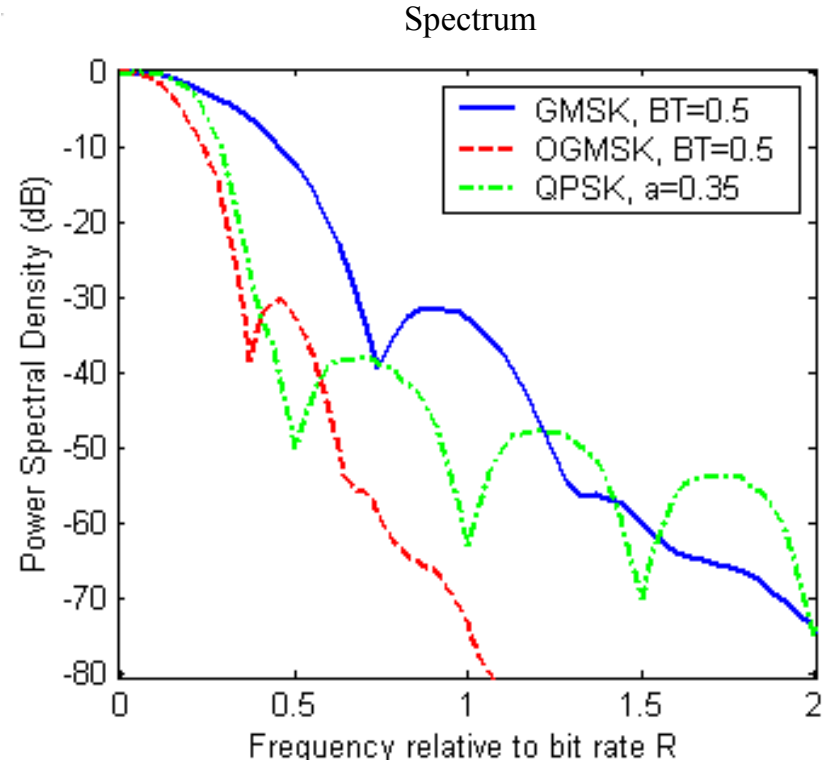

(b)

Fig. 2.7 Power spectra of MSK, OMSK/MAMSK and QPSK (a) Without pulse shaping (b) With pulse shaping 
Since the MAMSK signal can be considered as a sum of two MSK signals its spectral efficiency is twice that of MSK. However, it loses the constant envelope property due to multilevel modulation. Therefore MAMSK should be compared with QPSK rather than MSK. The spectral efficiencies of the three modulations are shown in Fig. 2.7. It is seen that the spectral efficiency of OMSK/MAMSK is better than QPSK, however, most of this advantage is lost after pulse shaping. Furthermore, there is no significant degradation in bit error performance of QPSK modulation with root raised cosine filtering (none in absence of timing jitter). However, the performance of OMSK/MAMSK with Gaussian pulse shaping degrades appreciably due to inter-symbol interference.

\subsection{Simulation Results}

The error performance of the modulation schemes was evaluated through Monte Carlo simulations the results of which are shown in Figs. 2.8-2.12. It can be seen from Fig. 2.8 that the error performance of OMSK is much worse than MSK.

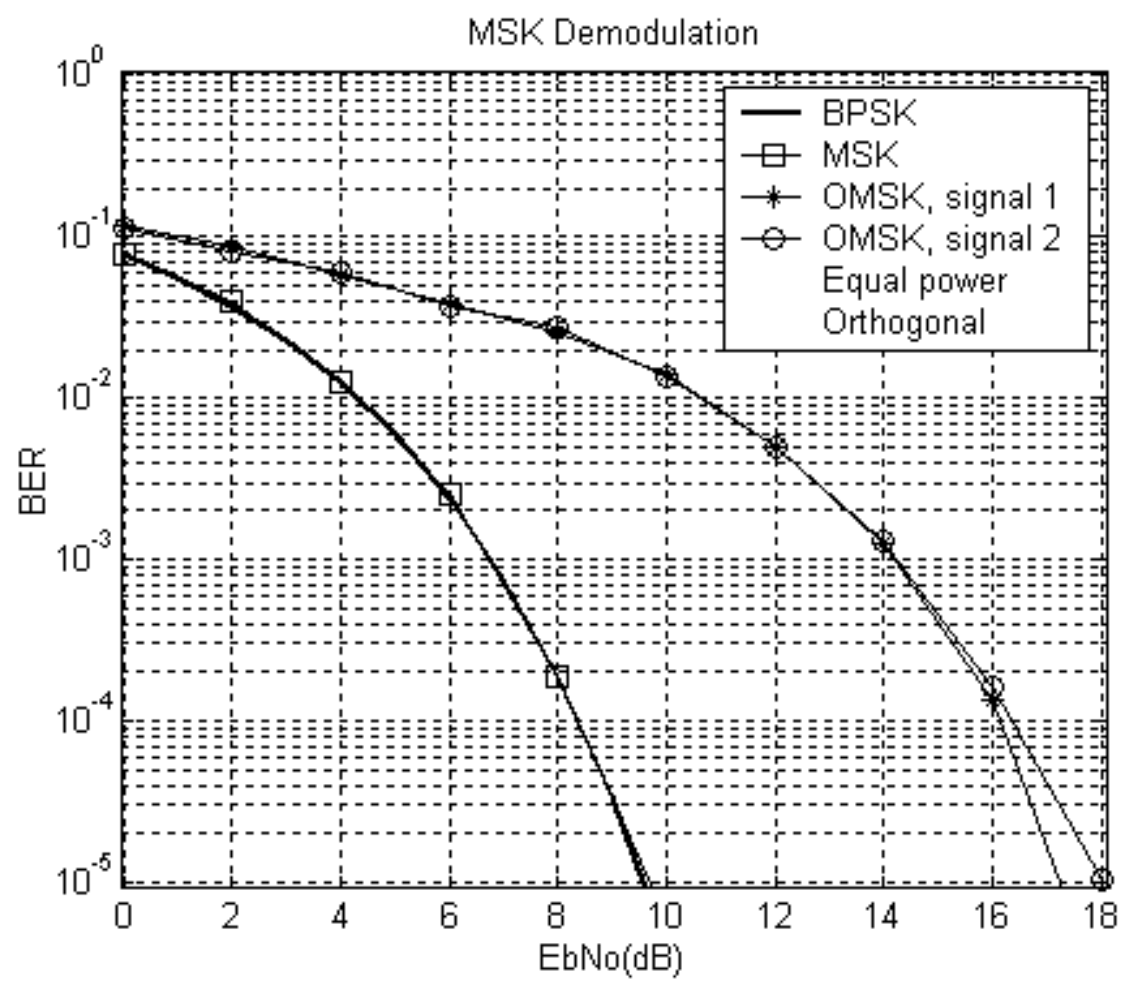

Fig. 2.8 Error performance of MSK signal in an AWGN channel with and without interference 
This might be due to some cross-interfering terms that we have neglected in our analysis. Since we are more interested in the case when one of the signals is an interferer we next evaluate the performance for the case when the signals are not orthogonal. The results are shown in Fig. 2.9. As expected the performance degrades as the deviation from orthogonality increases.

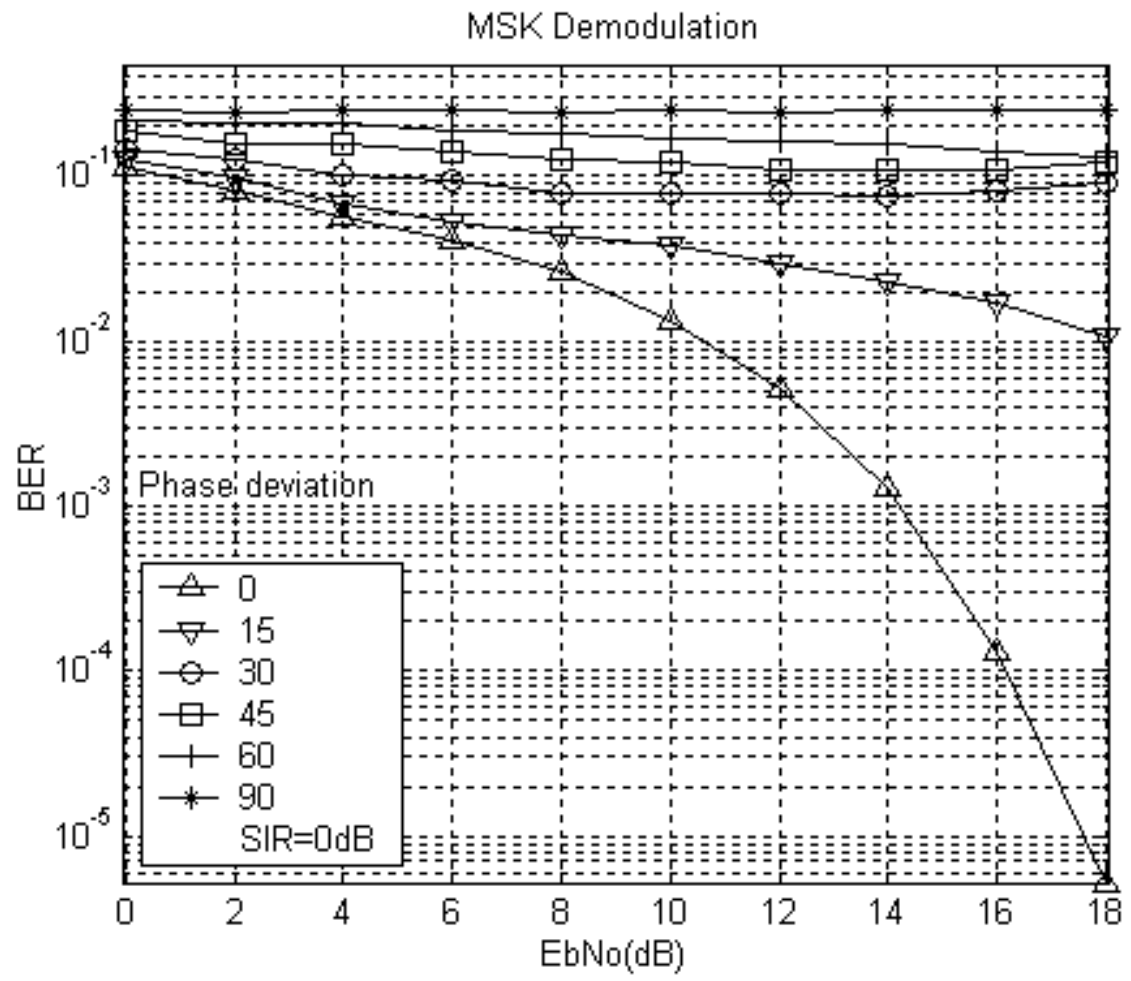

Fig. 2.9 Error performance when the interference is not orthogonal $(\mathrm{SIR}=0 \mathrm{~dB})$

To get a better understanding of the influence of phase difference on the bit error rate (BER), the BER is plotted as a function of phase deviation from orthogonality. The results are shown in Figs. 2.10 and 2.11. As expected the performance degrades as the phase deviation increases. One interesting point to be noticed is that for the high SNR and SIR greater than zero there is slight improvement in performance as the phase deviation increases from 60 to 90 degrees. Therefore for high SNR and SIR greater than zero the most undesirable case is not when phase deviation is 90 degrees (signals are co-phase) rather it is when the phase deviation is 60 (or 120) degrees. 


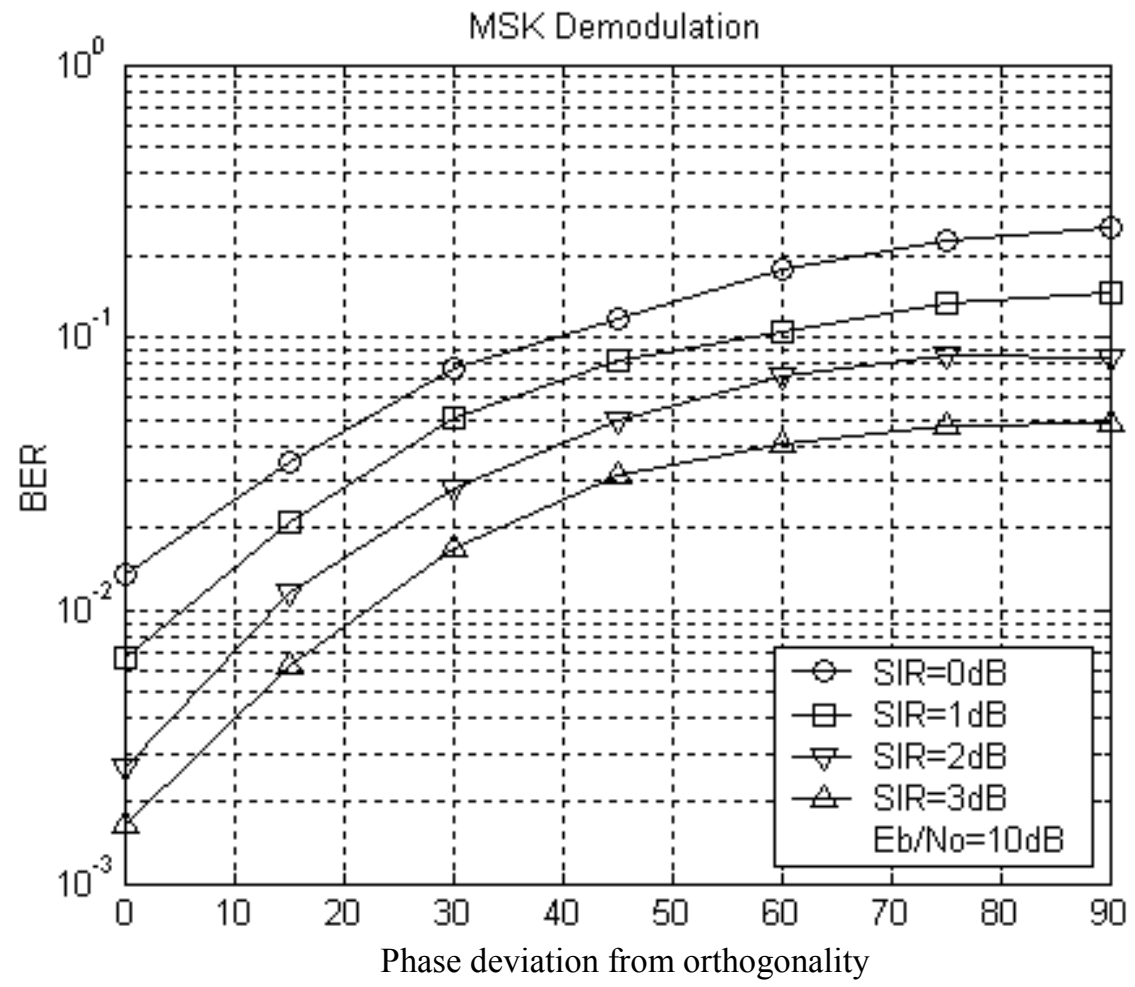

Fig. 2.10 Error performance for various SIRs and phase deviations, $\mathrm{Eb} / \mathrm{No}=10 \mathrm{~dB}$

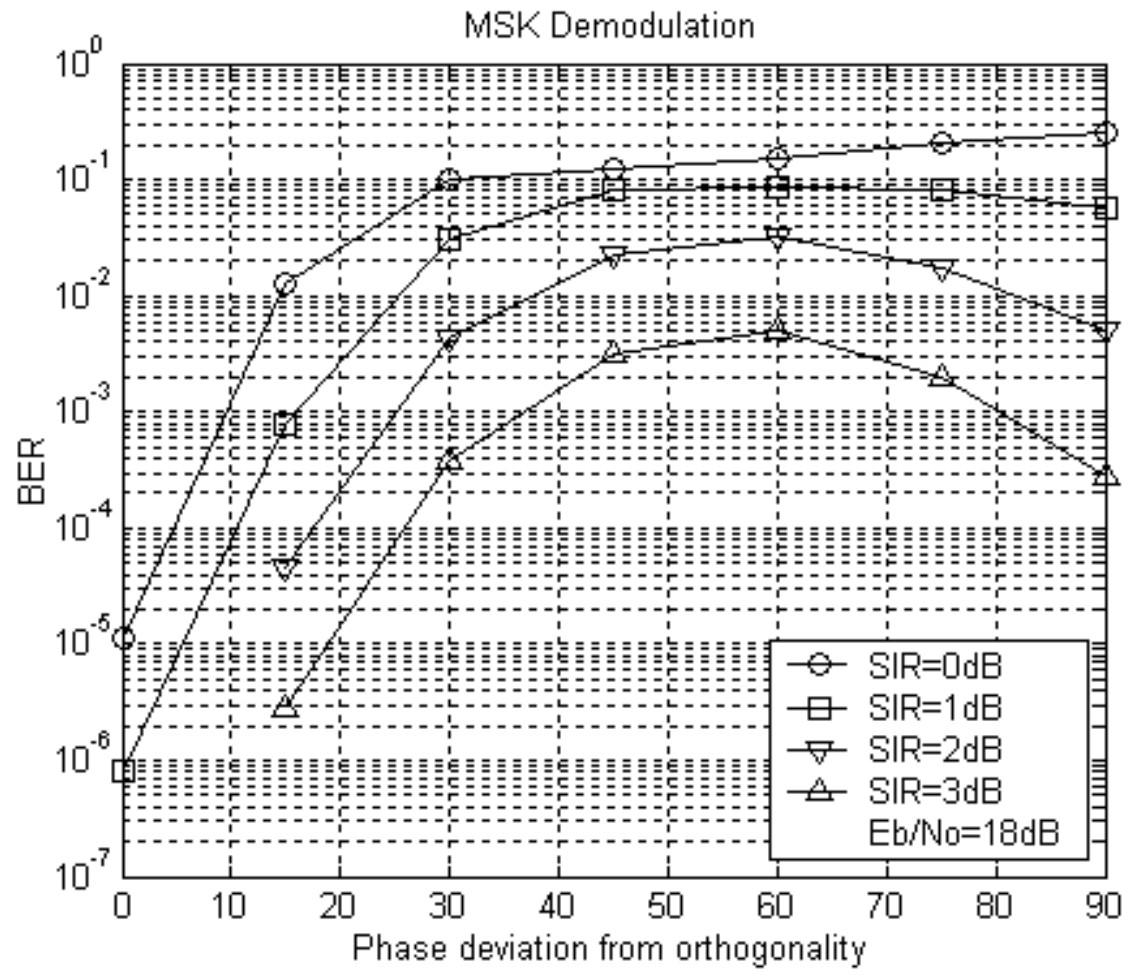

Fig. 2.11 Error performance for various SIRs and phase deviations, $\mathrm{Eb} / \mathrm{No}=18 \mathrm{~dB}$ 


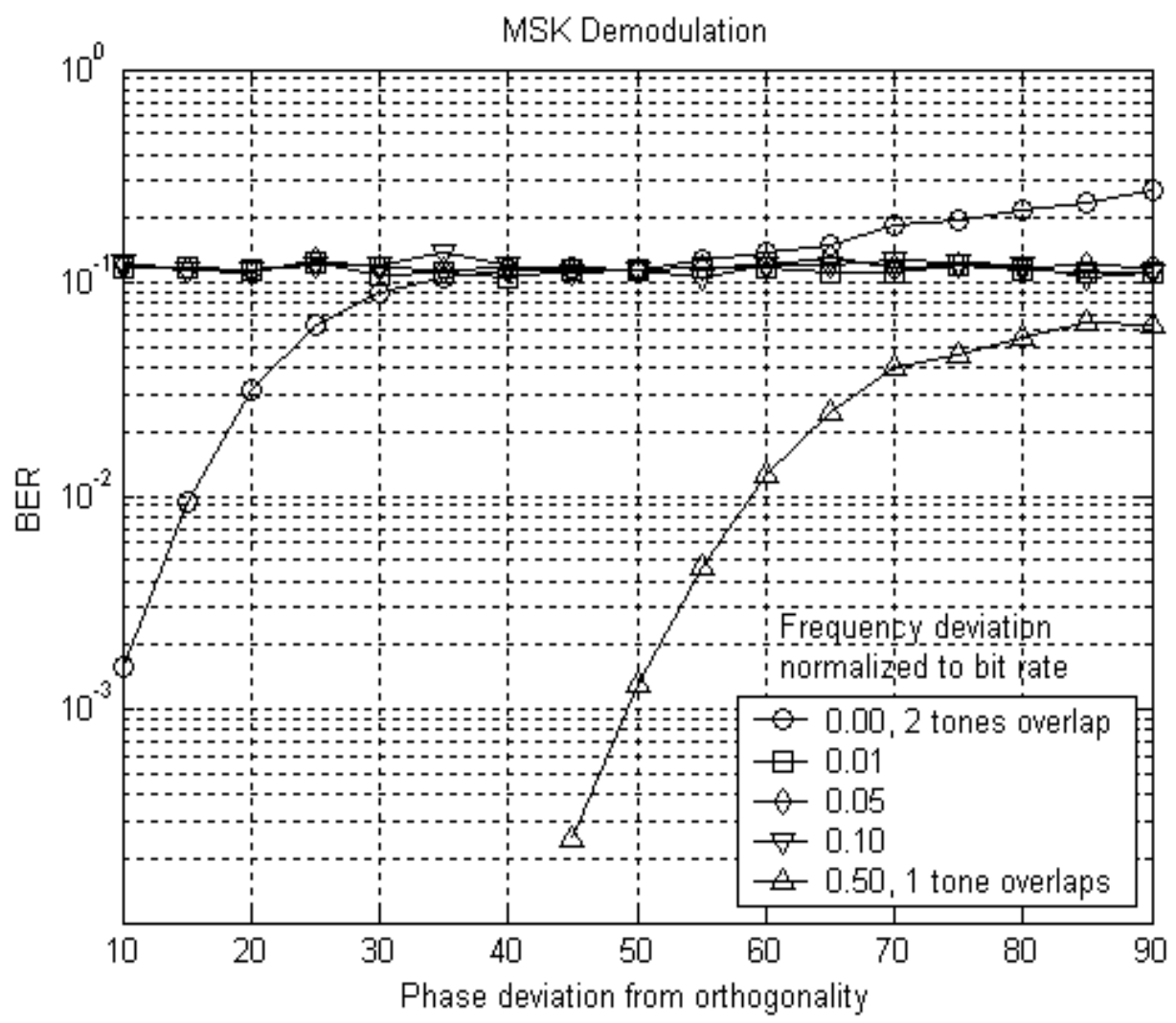

Fig. 2.12 Error performance for different values of frequency separation $(\mathrm{SIR}=0 \mathrm{~dB})$

Finally the error performance was evaluated for the case when the two signals do not have the same carrier frequencies. It was observed that the performance of the correlation receiver actually degrades for small difference in frequencies. Improvement is only obtained when the difference in carrier frequencies approaches 0.5 times the bit rate. This is an expected result as it corresponds to the case when one of the two tones overlaps as shown in Fig. 2.13(b).

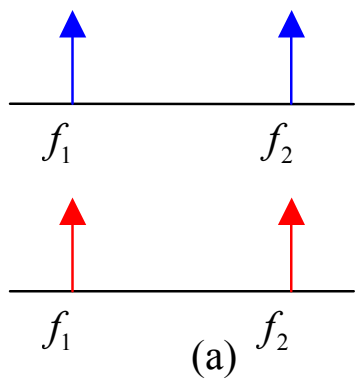

Fig. 2.13
Signal 1

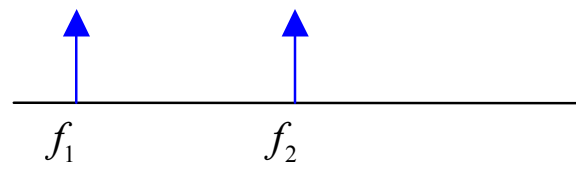

Signal 2

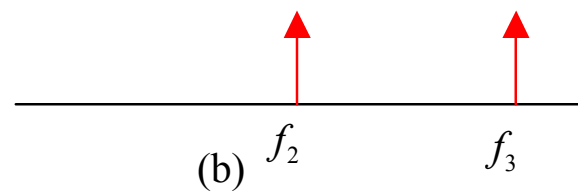

(a) Both tones overlap (b) One tone overlaps 


\subsection{Summary}

In this chapter we discussed the problem of co-channel interference in wireless communications with reference to MSK signals. A special case was studied when the two MSK signals are orthogonal to each other and a receiver architecture was proposed. It was noted that a multi-level MSK signal is in fact a special case of multi-amplitude minimum shift keying (MAMSK). MAMSK is a spectrally efficient modulation scheme however it is not constant envelope. If a non-constant envelope modulation scheme is to be used, QPSK would be preferred over MAMSK because QPSK with RRC filtering gives a reasonably good spectral efficiency with error performance that is superior to MAMSK. Finally simulation results were presented that showed the effect of phase deviation from orthogonality on the error performance. The case of different carrier frequencies was also simulated and it was found that the BER actually degrades with small difference in carrier frequencies and the advantage is only obtained when the difference is equal to 0.5 times the bit-rate. This corresponds to the condition when only one of the 2 MSK tones overlaps in the frequency domain. 


\section{Chapter III Minimum Variance Unbiased Estimation}

\subsection{Introduction}

In this chapter we study the problem of parameter estimation for the case in which the signal models are known. The signal models are assumed to be linear and this allows us to find optimal estimators using the properties of the Cramer-Rao lower bound (CRLB). This approach allows us to set a bound on the performance that we can expect to achieve when the signal models are unknown or when it is difficult to represent them by a linear model. The latter problem is considered in the next chapter.

\subsection{The Estimation Problem}

The problem of estimation is to determine an unknown parameter $\theta$ from a discrete-time waveform $\boldsymbol{x}=\{x[0], x[1], \ldots, x[N-1]\}$ which depends upon $\theta$. This has applications in many fields including image and speech processing, biomedicine, seismology and communications. In communications the received waveform might be a carrier that is amplitude, phase or frequency modulated by the parameter $\theta$ and is corrupted by the channel noise. The task of a communications receiver is to estimate the value of $\theta$ based upon the received waveform. This is essentially the definition of an estimator. Formally "An estimator is a rule that assigns a value to $\theta$ based upon the observations" [Kay93]. Mathematically an estimator can be defined as

$$
\hat{\theta}=g(x[0], x[1], \ldots, x[N-1])
$$

where $g$ is some function. The first step in determining a good estimator is to describe the data by a mathematical model. Since the data is random in nature it is described by its 
probability density function (PDF) $p(x[0], x[1], \ldots, x[N-1] ; \theta)$. This is a set of PDFs that depends upon the parameter $\theta$, i.e., we have a different PDF for each value of $\theta$. If $s[n ; \theta]$ represents the signal model and $x[\mathrm{n}]$ is the received data sequence then mathematically

$$
x[n]=s[n ; \theta]+w[n] \quad n=0,1, \ldots, N-1
$$

where $w[\mathrm{n}]$ is an additive white Gaussian noise (AWGN) process with PDF $N\left(0, \sigma^{2}\right)$. The probability density function of $x[\mathrm{n}]$ conditioned on the unknown parameter $\theta$, $p(x[\mathrm{n}] ; \theta)$, can then be written as

$$
p(x[n] ; \theta)=\frac{1}{(2 \pi \sigma)^{N / 2}} \exp \left[-\frac{1}{2 \sigma^{2}} \sum_{n=0}^{N-1}(x[n]-s[n ; \theta])^{2}\right]
$$

This is called the likelihood function. When the estimation problem is defined as in (3.3), it is referred to as Classical Estimation. Here the parameter to be estimated is assumed to be deterministic but unknown. This is different from Bayesian Estimation where the parameter to be estimated is assumed to be a random variable with known PDF.

\subsubsection{Minimum Variance Unbiased Estimation}

Now that we have defined the mathematical model for the estimation problem we need to find criteria for assessing the performance of different estimators. A natural choice is to choose an estimator that minimizes the mean square error (MSE), defined as

$$
\begin{aligned}
\operatorname{mse}(\hat{\theta}) & =E\left[(\hat{\theta}-\theta)^{2}\right] \\
& =E\left\{[(\hat{\theta}-E(\hat{\theta}))+(E(\hat{\theta})-\theta)]^{2}\right\} \\
& =\operatorname{var}(\hat{\theta})+[E(\hat{\theta})-\theta]^{2} \\
& =\operatorname{var}(\hat{\theta})+(\operatorname{bias}(\theta))^{2}
\end{aligned}
$$


It can be seen that the MSE takes into account the variance of the estimator as well as its bias. Any criterion that tries to minimize the bias generally results in an unrealizable estimator [Kay93]. The usual approach to this problem is to constrain the estimator to be unbiased and then find the one with the minimum variance. Such an estimator is called the minimum variance unbiased (MVU) estimator.

There are several methods for finding the MVU estimator. The most common ones are using Cramer-Rao lower bound (CRLB) and using the Rao-Blackwell-Lehmann-Scheffe (RBLS) theorem [Kay93]. We will concentrate on CRLB as it can be easily determined and once it has been determined it is evident whether an MVU exists or not.

\subsubsection{Cramer-Rao lower bound}

The Cramer-Rao lower bound (CRLB) is a fundamental bound in estimation theory that gives a reference with which the performance of any estimator can be compared. It is usually used in signal processing feasibility studies to determine the best results that can be expected for any given problem.

If the PDF $p(x ; \theta)$ satisfies the regularity condition

$$
E\left[\frac{\partial \ln p(x ; \theta)}{\partial \theta}\right]=0 \quad \text { for all } \theta
$$

where the expectation is taken with respect to $p(x ; \theta)$. The bound on the variance of an unbiased estimator is then given as

$$
\operatorname{var}(\hat{\theta}) \geq \frac{1}{-E\left[\frac{\partial^{2} \ln p(x ; \theta)}{\partial \theta^{2}}\right]}
$$


where the derivative is taken at the true value of $\theta$ and the expectation is taken with respect to $p(\boldsymbol{x} ; \theta)$. An estimator that satisfies this bound with equality exists if and only if the derivative of the log-likelihood function can be factorized as follows

$$
\frac{\partial \ln p(\boldsymbol{x} ; \theta)}{\partial \theta}=I(\theta)(g(\boldsymbol{x})-\theta)
$$

for some functions $g$ and $I$. The estimator that satisfies the CRLB is given as $\hat{\theta}=g(x)$ and its variance is equal to $1 / I(\theta)$. It must be noted that an estimator that satisfies the CRLB is an MVU but an MVU may or may not satisfy the CRLB and there is no turnthe-crank method for finding an MVU that does not satisfy the CRLB.

\subsubsection{Linear Models}

Finding an MVU estimator is in general a difficult task. However, if the data can be represented in linear model form the MVU estimator can be easily determined using the properties of the CRLB. This is a very important result because many digital communication signals can be represented by a linear model. The signal parameters contain the information that needs to be transmitted, therefore, as estimation accuracy improves the information reliability also improves. The linear model can be written in matrix notation as

$$
\boldsymbol{x}=\boldsymbol{H \theta}+\boldsymbol{w}
$$

where

$$
\begin{aligned}
\boldsymbol{x} & =\left[\begin{array}{llll}
x[0] & x[1] & \ldots & x[N-1
\end{array}\right]^{T} \\
\boldsymbol{w} & =\left[\begin{array}{llll}
w[0] & w[1] & \ldots & w[N-1
\end{array}\right]^{T} \\
\boldsymbol{\theta} & =\left[\begin{array}{llll}
\boldsymbol{\theta}_{1} & \boldsymbol{\theta}_{2} & \ldots & \boldsymbol{\theta}_{p}
\end{array}\right]^{T}
\end{aligned}
$$


where matrix $\boldsymbol{H}$ is a known $N \times \mathrm{x} p$ matrix (where $N>p$ and the rank of $H$ is $p$ ) and is normally referred to as the observation matrix, $\boldsymbol{x}$ is an $N \mathrm{x} 1$ vector of observations, $\boldsymbol{\theta}$ is a $p \times 1$ vector of parameters to be estimated and $\boldsymbol{w}$ is an $N \times 1$ noise vector with PDF $N\left(0, \sigma^{2} \boldsymbol{I}\right)$. The observation matrix $\boldsymbol{H}$ and the vector $\boldsymbol{\theta}$ completely define the signal. Here the noise has been assumed to be Gaussian but it can be generalized to include other types of noise as well.

It can shown that for the linear model given by (3.8) we have [Kay93]

$$
\frac{\partial \ln p(\boldsymbol{x} ; \boldsymbol{\theta})}{\partial \boldsymbol{\theta}}=\frac{1}{\sigma^{2}}\left[\boldsymbol{H}^{T} \boldsymbol{x}-\boldsymbol{H}^{T} \boldsymbol{H} \boldsymbol{\theta}\right]
$$

or

$$
\frac{\partial \ln p(\boldsymbol{x} ; \boldsymbol{\theta})}{\partial \boldsymbol{\theta}}=\frac{\boldsymbol{H}^{T} \boldsymbol{H}}{\sigma^{2}}\left[\left(\boldsymbol{H}^{T} \boldsymbol{H}\right)^{-1} \boldsymbol{H}^{T} \boldsymbol{x}-\boldsymbol{\theta}\right]
$$

Comparing (3.10) with (3.7) we find that the MVU estimator for a linear model is

$$
\hat{\boldsymbol{\theta}}=g(x)=\left(\boldsymbol{H}^{T} \boldsymbol{H}\right)^{-1} \boldsymbol{H}^{T} \boldsymbol{x}
$$

and its covariance matrix is

$$
\boldsymbol{C}_{\hat{\theta}}=\boldsymbol{I}^{-1}(\theta)=\sigma^{2}\left(\boldsymbol{H}^{T} \boldsymbol{H}\right)^{-1}
$$

The MVU estimator given by (3.11) attains the Cramer-Rao lower bound and hence is said to be efficient. It must be noted the matrix $\left(\boldsymbol{H}^{T} \boldsymbol{H}\right)$ might not always be invertible. However for most practical applications the matrix $\boldsymbol{H}$ has columns that are linearly independent and this guarantees the invertability of $\left(\boldsymbol{H}^{T} \boldsymbol{H}\right)$. 


\subsection{Minimum Variance Unbiased Estimator for Narrowband Signals}

We have shown in the last section that the key to finding an efficient estimator is to model the signal in linear form. Fortunately, the linear model can represent a broad class of communication signals. A typical narrowband communication signal can be written in quadrature form as

$$
s(t)=a \cos \left(2 \pi f_{c} t\right)+b \sin \left(2 \pi f_{c} t\right)
$$

where $f_{c}$ is the known carrier frequency and parameters $a$ and $b$ contain the information to be transmitted. The values of $a$ and $b$ have to be estimated at the receiver given the noisy observation of $s(t)$. The received signal, $\mathrm{x}(t)$, can be written as

$$
x(t)=s(t)+w(t)
$$

Suppose that we have $N$ samples of the received signal $x(t)$. Therefore the sampled received signal, $x[\mathrm{n}]$, is

$$
x[n]=s[n]+w[n] \quad n=0,1, \ldots N-1
$$

or

$$
x[n]=a \cos \left(2 \pi \frac{k}{N} n\right)+b \sin \left(2 \pi \frac{k}{N} n\right)+w[n]
$$

where $k \leq N / 2-1$. This can be written in the familiar form for a linear model as

$$
\boldsymbol{x}=\boldsymbol{H \theta}+\boldsymbol{w}
$$

where 


$$
\boldsymbol{H}=\left[\begin{array}{cc}
1 & 0 \\
\cos 2 \pi \frac{k}{N} & \sin 2 \pi \frac{k}{N} \\
\vdots & \vdots \\
\cos 2 \pi \frac{k}{N}(N-1) & \sin 2 \pi \frac{k}{N}(N-1)
\end{array}\right] \quad \text { and } \quad \theta=\left[\begin{array}{l}
a \\
b
\end{array}\right]
$$

The MVU estimator for the linear model given in (3.17) is [Kay93]

$$
\hat{\boldsymbol{\theta}}=\left(\boldsymbol{H}^{T} \boldsymbol{H}\right)^{-1} \boldsymbol{H}^{T} \boldsymbol{x}
$$

Let the columns of the observation matrix $\boldsymbol{H}$ be represented by $\boldsymbol{h}_{1}$ and $\boldsymbol{h}_{2}$. Therefore

$$
H=\left[\begin{array}{ll}
h_{1} & h_{2}
\end{array}\right]
$$

and

$$
\boldsymbol{H}^{T} \boldsymbol{H}=\left[\begin{array}{ll}
\boldsymbol{h}_{\boldsymbol{1}}{ }^{T} \boldsymbol{h}_{\boldsymbol{1}} & \boldsymbol{h}_{1}{ }^{T} \boldsymbol{h}_{\mathbf{2}} \\
\boldsymbol{h}_{\mathbf{2}}{ }^{T} \boldsymbol{h}_{\mathbf{1}} & \boldsymbol{h}_{\mathbf{2}}{ }^{T} \boldsymbol{h}_{\mathbf{2}}
\end{array}\right]
$$

It can be shown that non-diagonal elements of the above matrix are equal to zero. This is due to fact that the columns of matrix $\boldsymbol{H}$ are orthogonal. Therefore $\boldsymbol{H}^{T} \boldsymbol{H}$ is a diagonal matrix that can be easily inverted. Substituting the values in (3.11) we have

$$
\begin{aligned}
& \hat{\boldsymbol{\theta}}=\left(\boldsymbol{H}^{T} \boldsymbol{H}\right)^{-1} \boldsymbol{H}^{T} \boldsymbol{x} \\
& \hat{\boldsymbol{\theta}}=\frac{2}{N} \boldsymbol{H}^{T} \boldsymbol{x} \\
& \hat{\boldsymbol{\theta}}=\frac{2}{N}\left[\begin{array}{l}
\boldsymbol{h}_{1}{ }^{T} \\
\boldsymbol{h}_{2}{ }^{T}
\end{array}\right] \boldsymbol{x}=\left[\begin{array}{l}
\hat{a} \\
\hat{b}
\end{array}\right]
\end{aligned}
$$




$$
\begin{aligned}
& \hat{a}=\frac{2}{N} \sum_{n=0}^{N-1} x[n] \cos \left(2 \pi \frac{k}{N} n\right) \\
& \hat{b}=\frac{2}{N} \sum_{n=0}^{N-1} x[n] \sin \left(2 \pi \frac{k}{N} n\right)
\end{aligned}
$$

It is obvious that (3.18) and (3.19) represent the typical correlation receiver shown in Fig. 3.1. The observed signal $\boldsymbol{x}$ is multiplied by the basis functions $\boldsymbol{h}_{1}$ and $\boldsymbol{h}_{2}$ and then summation is performed over $N$ samples. It must be noted that in this case $N$ is selected to be equal to the number of samples per bit. Therefore it can be concluded that the MVU estimator for the parameters $a$ and $b$ is a correlation receiver.

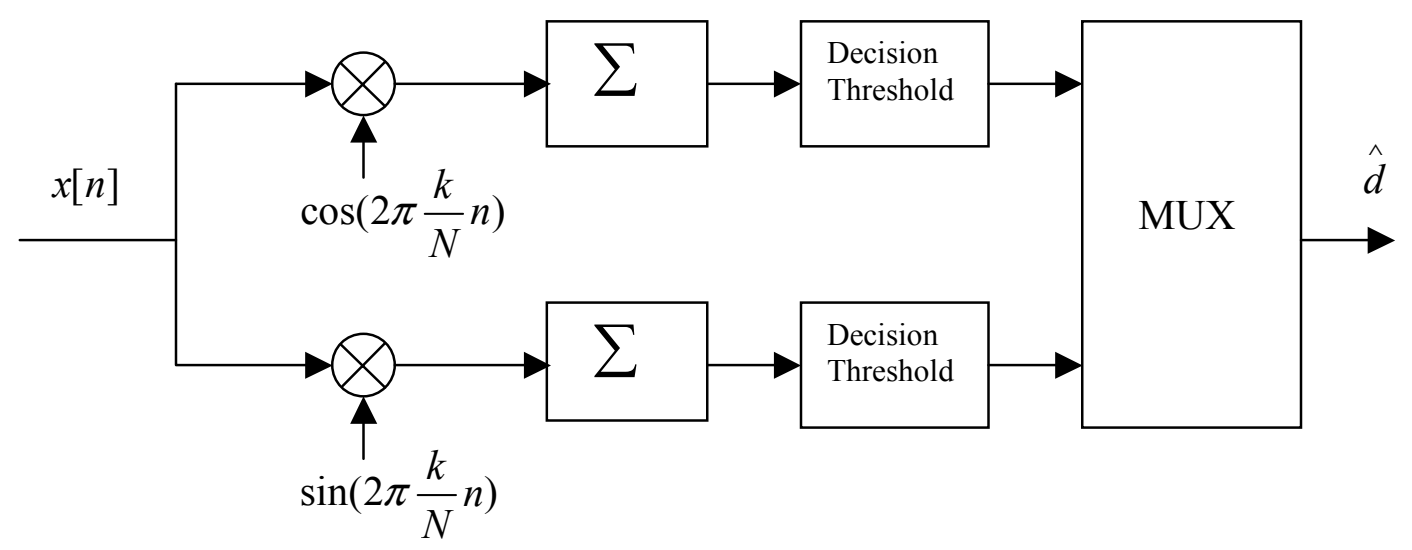

Fig. 3.1 Correlation receiver

The above discussion can be extended to include MSK type signals. The only difference will be that the columns of matrix $\boldsymbol{H}$ (basis functions) will be

$$
\begin{aligned}
& \boldsymbol{h}_{1}=\cos \left(2 \pi \frac{k_{1}}{N} n\right) \cos \left(2 \pi \frac{k_{2}}{N} n\right) \\
& \boldsymbol{h}_{2}=\sin \left(2 \pi \frac{k_{1}}{N} n\right) \sin \left(2 \pi \frac{k_{2}}{N} n\right)
\end{aligned}
$$


where $k_{1}=0.5$ and $k_{2} \leq N / 2-1$ (usually $k_{2} \gg k_{1}$ ). The MVU estimator for the MSK signal results in a parallel receiver architecture similar to the one shown in Fig. 3.1. It should be noted however, that only one of the two parameters is used for making each bit decision.

It can be shown that whenever the columns of $\boldsymbol{H}$ (basis functions) are orthogonal $\boldsymbol{H}^{T} \boldsymbol{H}$ is a diagonal matrix that is easily invertible and the resulting architecture is a typical correlation receiver. However if the columns of $\boldsymbol{H}$ are not orthogonal $\boldsymbol{H}^{T} \boldsymbol{H}$ is not a diagonal matrix and the exact solution is given by (3.11). This might be the case in narrowband communication systems where the carriers lose their orthogonality due to mismatch between local oscillators (LO). The signal constellation of a QPSK signal is shown in Fig. 3.2. It is seen that the constellation gets skewed due to the LO mismatch. It therefore seems intuitive to use an MVU estimator to nullify the effect of interference between the carriers. The results are shown in Fig. 3.3 and 3.4. It is seen that for a phase deviation of 10 degrees the performance of the MVU matches closely with the ideal performance and for a phase deviation of 20 degrees there is a loss of about $1 \mathrm{~dB}$. However in both the cases the MVU performance is much better than that of the matched filter. It must be noted that we have assumed perfect knowledge of LO phase mismatch at the receiver.

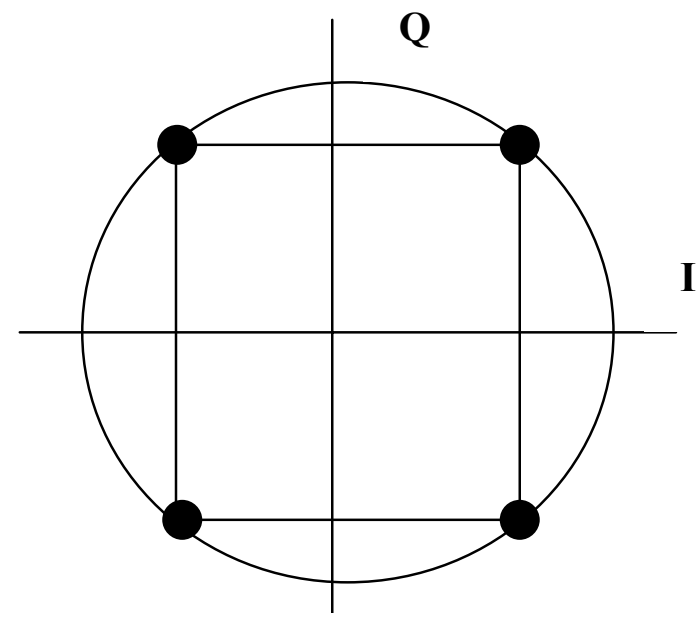

(a)

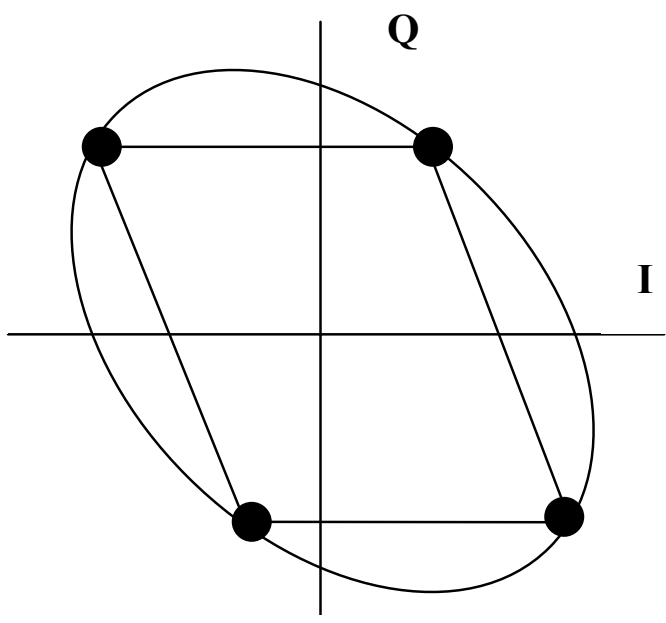

(b)

Fig. 3.2 Signal constellation of a QPSK signal (a) ideal (b) skewed 


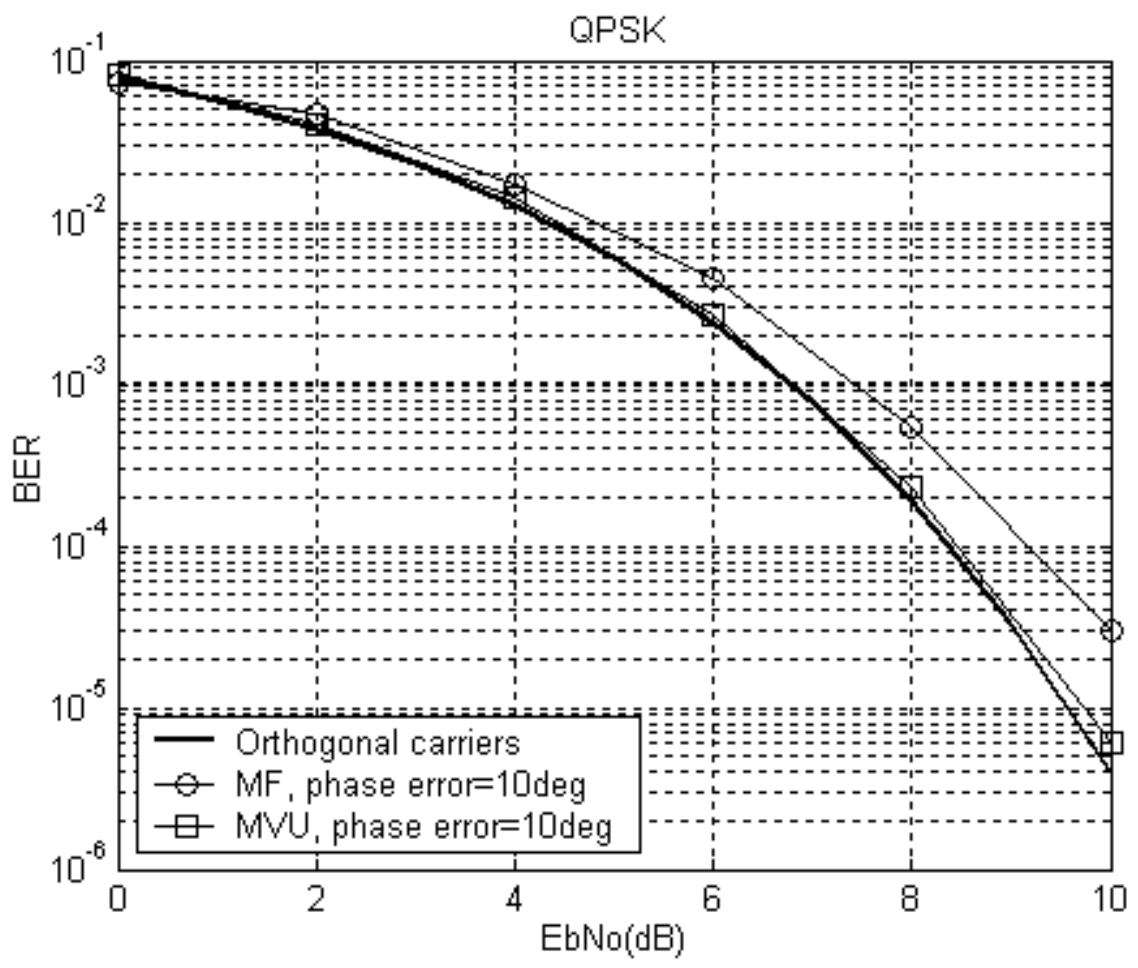

Fig. 3.3. Comparison of MF and MVU for non-orthogonal carriers. Phase error $=10 \mathrm{deg}$

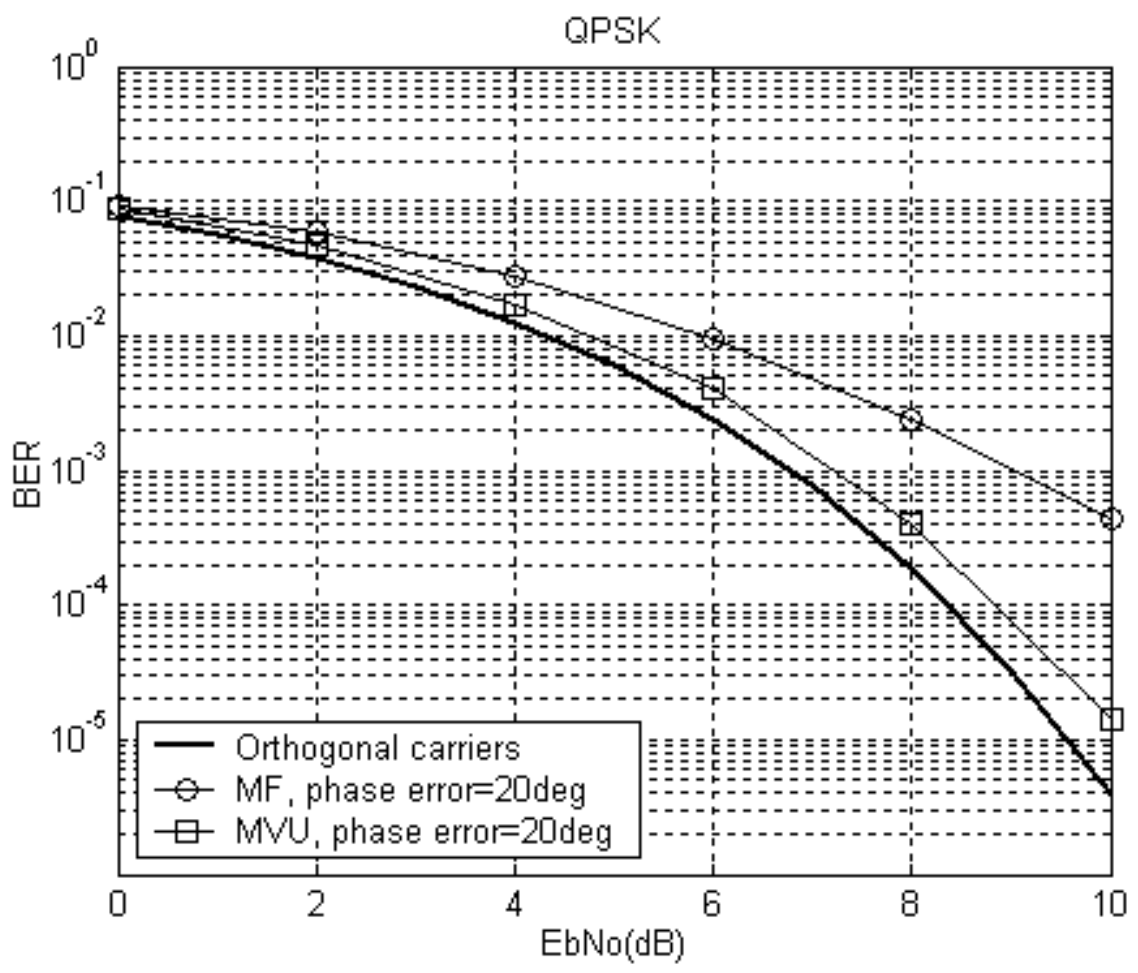

Fig. 3.4. Comparison of MF and MVU for non-orthogonal carriers. Phase error $=20 \mathrm{deg}$ 
In chapter 2 it was shown that with proper phase offset two MSK signals are orthogonal to each other, therefore, we can treat the problem of co-channel interference of MSK signals as a case of non-orthogonal carriers and can use the linear modeling approaches already discussed. In this case $\boldsymbol{H}$ is a rank 4 matrix and $\boldsymbol{H}^{T} \boldsymbol{H}$ is a $4 \times 4$ matrix. The columns of $\boldsymbol{H}$ are defined as

$$
\begin{gathered}
\boldsymbol{h}_{1}=\cos \left(2 \pi \frac{k_{1}}{N} n\right) \cos \left(2 \pi \frac{k_{2}}{N} n\right) \\
\boldsymbol{h}_{2}=\sin \left(2 \pi \frac{k_{1}}{N} n\right) \sin \left(2 \pi \frac{k_{2}}{N} n\right) \\
\boldsymbol{h}_{3}=\cos \left(2 \pi \frac{k_{1}}{N} n\right) \cos \left(2 \pi \frac{k_{2}}{N} n+\phi\right) \\
\boldsymbol{h}_{4}=\sin \left(2 \pi \frac{k_{1}}{N} n\right) \sin \left(2 \pi \frac{k_{2}}{N} n+\phi\right)
\end{gathered}
$$

and

$$
\boldsymbol{H}^{T} \boldsymbol{H}=\left[\begin{array}{llll}
\boldsymbol{h}_{1}{ }^{T} h_{1} & h_{1}{ }^{T} h_{2} & h_{1}{ }^{T} h_{3} & h_{1}{ }^{T} h_{4} \\
\boldsymbol{h}_{2}{ }^{T} h_{1} & h_{2}{ }^{T} h_{2} & h_{2}{ }^{T} h_{3} & h_{2}{ }^{T} h_{4} \\
h_{3}{ }^{T} h_{1} & h_{3}{ }^{T} h_{2} & h_{3}{ }^{T} h_{3} & h_{3}{ }^{T} h_{4} \\
h_{4}{ }^{T} h_{1} & h_{4}{ }^{T} h_{2} & h_{4}{ }^{T} h_{3} & h_{4}{ }^{T} h_{4}
\end{array}\right]
$$

Here $\phi$ is the phase difference between the two MSK signals and, as shown in chapter 2, if $\phi=90^{\circ}$ then the two signals are perfectly orthogonal and the above matrix is simply a diagonal matrix. This results in a two-user correlation receiver for MSK signals. However if $\phi \neq 90^{\circ}$ then $\boldsymbol{H}^{T} \boldsymbol{H}$ is not an identity matrix and the correlation receiver is followed by a decorrelation operation. This results in receiver architecture similar to a multiuser detector for DS-SS signals that is explained in the next section. 
The effect of decorrelation operation can be understood by looking at the checkerboard plot of $\left(H^{T} H\right)$. It can be seen that as the phase deviation from orthogonality increases the non-diagonal terms, that represent interference, become stronger. When the two MSK signals are orthogonal (phase error $=0 \mathrm{deg}$ ) there are no interfering terms and when they have the same phase (phase error $=90 \mathrm{deg}$ ) the interfering terms become equal to the diagonal terms. Also note that there is only one interfering term in each row.
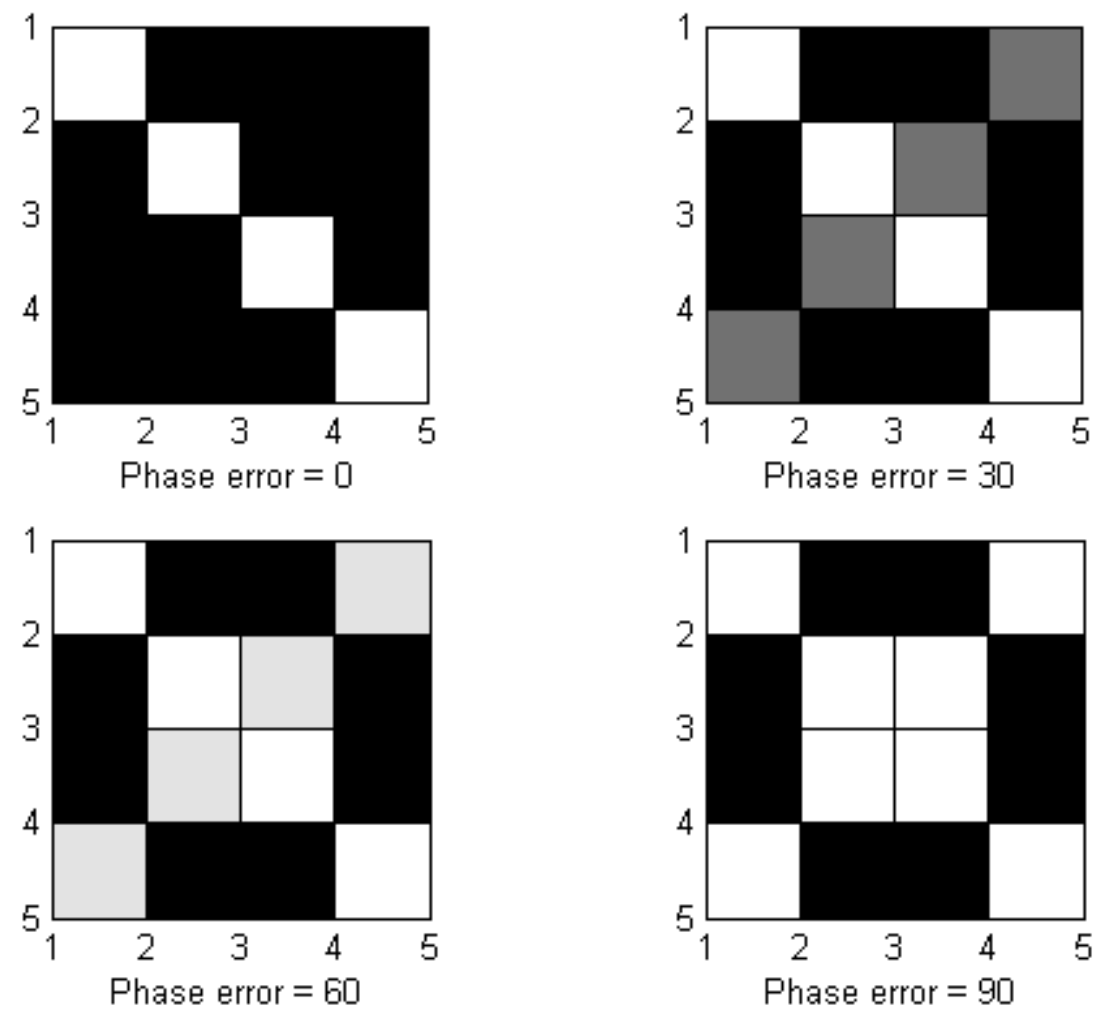

Fig. 3.5 Checkerboard plot of $\left(H^{T} H\right)$ for various phase error values. Lighter shades represent stronger values. White is strongest and black is zero

The matrix $\left(H^{T} H\right)^{-1}$ has the same structure as $\left(H^{T} H\right)$, however, the sign of the nondiagonal terms is opposite. The decorrelation operation strips off the interfering terms and demodulates the desired signal. It can be shown that the minimum mean square error (MMSE) estimator essentially performs the same operation for high signal to noise ratios. 


\subsection{Minimum Variance Unbiased Estimator for Wideband Signals}

So far we have restricted our attention to narrowband signals, however, the theory of minimum variance unbiased estimation can also be applied to spread spectrum signals. Spread spectrum signals can also be represented in linear model form and hence an MVU estimator can be found that satisfies the Cramer-Rao lower bound. We will consider direct sequence signals and use the baseband representation that is independent of the modulation scheme being used. This allows us to determine a general set of rules that can be applied whatever the modulation scheme may be. The signals are assumed to be symbol and chip synchronous. A spread spectrum signal can be defined as

$$
s[n]=A_{1} b_{1} s_{1}[n]+A_{2} b_{2} s_{2}[n]+\ldots+A_{K} b_{K} s_{K}[n]
$$

where $A_{k}$ is the amplitude, $b_{k} \in\{-1,+1\}$ is the information that needs to be transmitted and $s_{k}[n]$ is the spreading sequence of the $k$ th user. The signal observed at a receiver is given as

$$
x[n]=A_{1} b_{1} s_{1}[n]+A_{2} b_{2} s_{2}[n]+\ldots+A_{K} b_{K} s_{K}[n]+w[n]
$$

The above equation can be represented in linear model (3.8) form as

$$
\boldsymbol{x}=\boldsymbol{H} \boldsymbol{\theta}+\boldsymbol{w}
$$

where

$$
\boldsymbol{H}=\left[\begin{array}{llll}
\boldsymbol{s}_{1} & \boldsymbol{s}_{2} & \ldots & \boldsymbol{s}_{K}
\end{array}\right] \text { and } \boldsymbol{\theta}=\left[\begin{array}{lllll}
A_{1} b_{1} & A_{2} b_{2} & \ldots & A_{K} b_{K}
\end{array}\right]^{T}
$$

The task of the receiver is to estimate the sign of $A_{k} b_{k}$ (or simply $b_{k}$ ). For a linear model the MVU estimator is given by (3.11). Where 


$$
\boldsymbol{H}^{T} \boldsymbol{H}=\left[\begin{array}{c}
\boldsymbol{s}_{1}{ }^{T} \\
\boldsymbol{s}_{2}{ }^{T} \\
\vdots \\
\boldsymbol{s}_{K}{ }^{T}
\end{array}\right]\left[\begin{array}{llll}
\boldsymbol{s}_{1} & \boldsymbol{s}_{2} & \ldots & \boldsymbol{s}_{K}
\end{array}\right]
$$

or

$$
\boldsymbol{H}^{T} \boldsymbol{H}=\left[\begin{array}{cccc}
\boldsymbol{s}_{1}{ }^{\boldsymbol{T}} \boldsymbol{s}_{1} & \boldsymbol{s}_{1}{ }^{\boldsymbol{T}} \boldsymbol{s}_{2} & \ldots & \boldsymbol{s}_{1}{ }^{\boldsymbol{T}} \boldsymbol{s}_{K} \\
\boldsymbol{s}_{2}{ }^{\boldsymbol{T}} \boldsymbol{s}_{1} & \boldsymbol{s}_{2}{ }^{\boldsymbol{T}} \boldsymbol{s}_{2} & \ldots & \boldsymbol{s}_{2}{ }^{\boldsymbol{T}} \boldsymbol{s}_{K} \\
\vdots & \vdots & \ddots & \vdots \\
\boldsymbol{s}_{K}{ }^{\boldsymbol{T}} \boldsymbol{s}_{1} & \boldsymbol{s}_{K}{ }^{\boldsymbol{T}} \boldsymbol{s}_{2} & \ldots & \boldsymbol{s}_{K}{ }^{\boldsymbol{T}} \boldsymbol{s}_{K}
\end{array}\right]
$$

The matrix $\boldsymbol{H}^{T} \boldsymbol{H}$ is recognized as the correlation matrix, $\boldsymbol{R}$, of the spreading codes and $\boldsymbol{H}^{T} \boldsymbol{x}$ represents the correlation of the received signal with the spreading codes. Therefore (3.11) represents a correlation with the spreading codes (matched filtering) followed by a decorrelation operation. This is the typical decorrelating multiuser detector for a synchronous CDMA channel [Ver98] and is shown in Fig. 3.6.

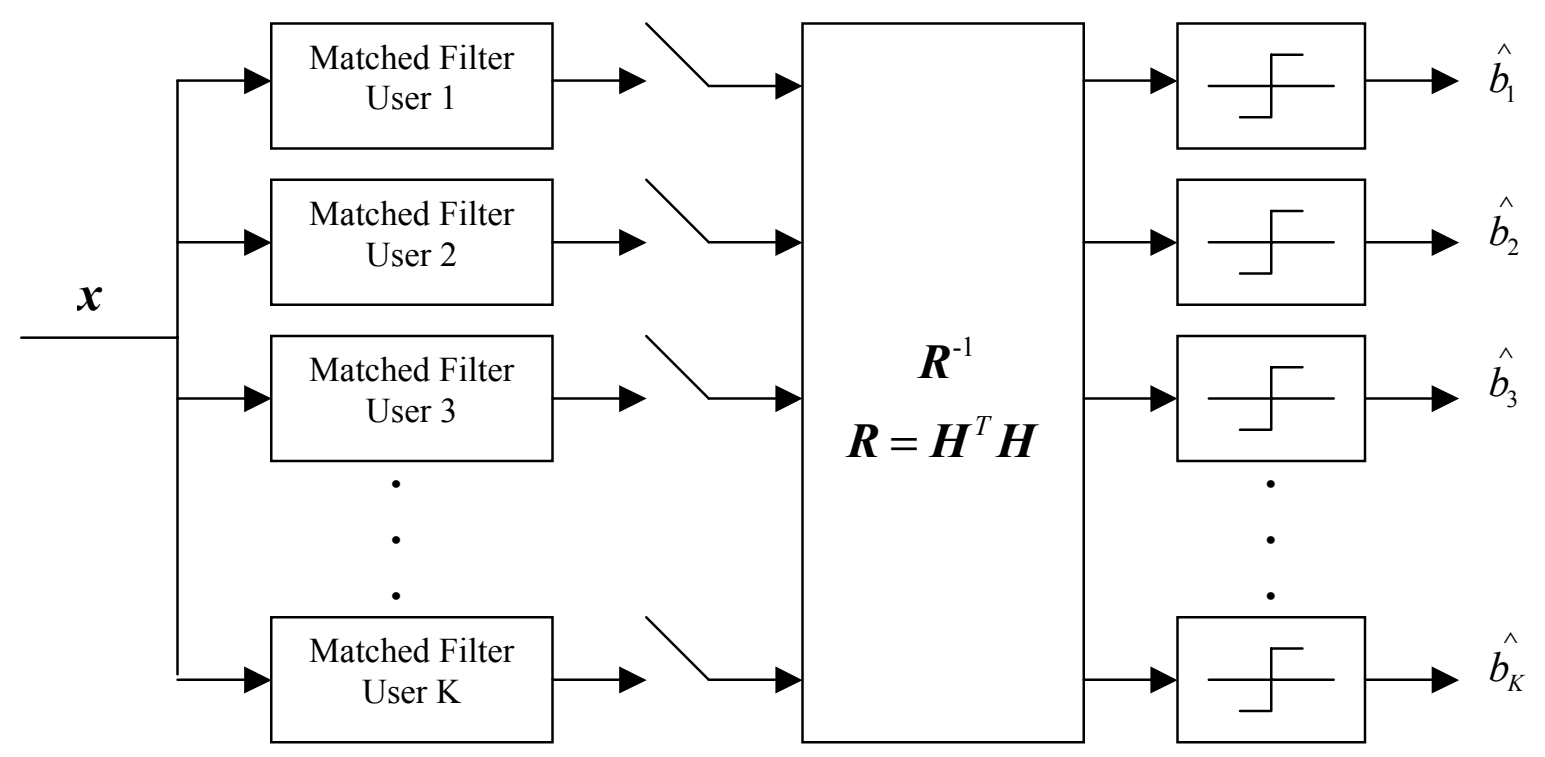

Fig. 3.6 Decorrelating detector for the synchronous channel 
Note that if the spreading codes are assumed to be perfectly orthogonal the matrix $\boldsymbol{R}$ is an identity matrix and the above receiver is simplified to a matched filter receiver. Also note that for the invertability of matrix $\boldsymbol{R}$ the columns of matrix $\boldsymbol{H}$ must be linearly independent. In most practical systems the number of users $K$ is large and this results in a very large correlation matrix (correlation matrix has dimensions $K \times K$ ). Therefore the direct inversion of matrix $\boldsymbol{R}$ is computationally very expensive. The inverse of matrix $\boldsymbol{R}$ is usually computed using some sub-optimal approaches.

\subsection{The Decorrelator}

Let us now examine the effect of the decorrelator in more detail. Consider the case when the number of users is equal to two i.e. $K=2$ and the received signal has not been corrupted by noise. Let us assume that correlation between the codes $s_{1}$ and $s_{2}$ is $\rho_{12}=\rho_{21}=\rho$. Therefore the matrix $\boldsymbol{R}$ or $\left(\boldsymbol{H}^{T} \boldsymbol{H}\right)$ can be written as

$$
\begin{aligned}
\boldsymbol{H}^{T} \boldsymbol{H} & =\left[\begin{array}{ll}
\boldsymbol{s}_{1}{ }^{T} \boldsymbol{s}_{1} & \boldsymbol{s}_{1}{ }^{T} \boldsymbol{s}_{2} \\
\boldsymbol{s}_{2}{ }^{T} \boldsymbol{s}_{1} & \boldsymbol{s}_{2}{ }^{T} \boldsymbol{s}_{2}
\end{array}\right] \\
& =\left[\begin{array}{ll}
1 & \rho \\
\rho & 1
\end{array}\right]
\end{aligned}
$$

and

$$
\left(\boldsymbol{H}^{T} \boldsymbol{H}\right)^{-1}=\frac{1}{1-\rho^{2}}\left[\begin{array}{cc}
1 & -\rho \\
-\rho & 1
\end{array}\right]
$$

and the matched filter outputs $y_{1}, y_{2}$ can be written as

$$
\boldsymbol{H}^{\boldsymbol{T}} \boldsymbol{x}=\left[\begin{array}{l}
y_{1} \\
y_{2}
\end{array}\right]=\left[\begin{array}{l}
\boldsymbol{s}_{1} \\
\boldsymbol{s}_{2}
\end{array}\right]\left[A_{1} b_{1} \boldsymbol{s}_{1}+A_{2} b_{2} \boldsymbol{s}_{2}\right]
$$


or

$$
\left[\begin{array}{l}
y_{1} \\
y_{2}
\end{array}\right]=\left[\begin{array}{l}
\mathrm{A}_{1} b_{1}+A_{2} b_{2} \rho_{12} \\
\mathrm{~A}_{1} b_{1} \rho_{21}+A_{2} b_{2}
\end{array}\right]
$$

Substituting (3.22) and (3.23) in (3.11) we have

$$
\hat{\boldsymbol{\theta}}=\left(\boldsymbol{H}^{T} \boldsymbol{H}\right)^{-1} \boldsymbol{H}^{T} \boldsymbol{x}=\frac{1}{1-\rho^{2}}\left[\begin{array}{cc}
1 & -\rho \\
-\rho & 1
\end{array}\right]\left[\begin{array}{l}
y_{1} \\
y_{2}
\end{array}\right]
$$

or

$$
\hat{\boldsymbol{\theta}}=\frac{1}{1-\rho^{2}}\left[\begin{array}{l}
y_{1}-\rho y_{2} \\
y_{2}-\rho y_{1}
\end{array}\right]
$$

Substituting the value of the match filter outputs from (3.23) in (3.24) we have

$$
\hat{\boldsymbol{\theta}}=\frac{1}{1-\rho^{2}}\left[\begin{array}{c}
A_{1} b_{1}+A_{2} b_{2} \rho-A_{1} b_{1} \rho^{2}-A_{2} b_{2} \rho \\
-A_{1} b_{1} \rho-A_{2} b_{2} \rho^{2}+A_{1} b_{1} \rho+A_{2} b_{2}
\end{array}\right]
$$

or

$$
\hat{\boldsymbol{\theta}}=\frac{1}{1-\rho^{2}}\left[\begin{array}{c}
A_{1} b_{1}-A_{1} b_{1} \rho^{2} \\
A_{2} b_{2}-A_{2} b_{2} \rho^{2}
\end{array}\right]
$$

or

$$
\hat{\boldsymbol{\theta}}=\left[\begin{array}{l}
A_{1} b_{1} \\
A_{2} b_{2}
\end{array}\right]
$$


It is seen that for the noiseless case the decorrelator removes the effect of correlation between the two codes from the matched filter outputs. Therefore it makes the perfect decision unlike the simple matched filter receiver that can make incorrect decisions even in the absence of noise (near-far problem). For the case when the received signal has been corrupted by noise there will be an additional term that is described in detail in the next section.

\subsection{Geometrical Interpretation of the Decorrelator}

The properties of the decorrelator have been studied extensively and several improvements and a geometrical interpretation have been given in [Jun96], [Ras96], [QLi00], [Eld02]. The decorrelator has been explained in the context of vector geometry and subspace concepts in matrix algebra. We will focus on the geometrical interpretation given by [Jun96] as it is intuitively appealing and also graphically comprehensible.

A conventional matched filter simply correlates the received signal $\boldsymbol{x}$ with the spreading code for each user $\boldsymbol{s}_{\boldsymbol{k}}$ to get the decision statistic $\hat{\theta}$. In matrix notation

$$
\hat{\theta}=\boldsymbol{H}^{T} \boldsymbol{x}
$$

or

$$
\hat{\theta}=\boldsymbol{H}^{T}(\boldsymbol{H} \theta+w)=\boldsymbol{H}^{T} \boldsymbol{H} \theta+H^{T} w
$$

This operation is shown graphically in Fig. 3.7 It is assumed that $A_{1}=1, A_{2}=1$ and $b_{1}=b_{2}=1$. It is seen that the matched filtering operation is simply the orthogonal projection of the received vector $\boldsymbol{x}$ onto the direction of the spreading codes. Since the spreading codes are not orthogonal the noise is no longer white and its covariance is $\boldsymbol{H}^{T} \boldsymbol{H}[$ Jun96]. 
The decorrelation operation can be written mathematically as

$$
\hat{\theta}=\left(\boldsymbol{H}^{T} \boldsymbol{H}\right)^{-1} \boldsymbol{H}^{T} \boldsymbol{x}
$$

or

$$
\hat{\theta}=\left(\boldsymbol{H}^{T} \boldsymbol{H}\right)^{-1} \boldsymbol{H}^{T}(\boldsymbol{H} \boldsymbol{\theta}+\boldsymbol{w})=\boldsymbol{\theta}+\left(\boldsymbol{H}^{T} \boldsymbol{H}\right)^{-1} \boldsymbol{H}^{T} \boldsymbol{w}
$$

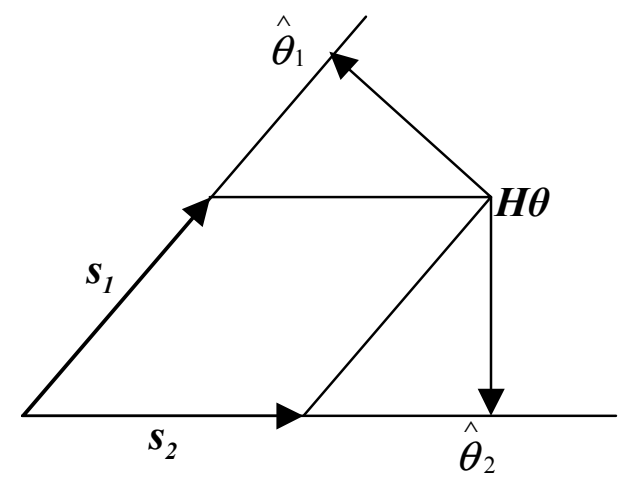

Fig. 3.7 Geometrical representation of matched filter for two users

To visualize the operation of the decorrelator we need to understand the properties of the inverse of a matrix. By definition the $i$ th row of matrix $\boldsymbol{H}^{T} \boldsymbol{H}^{-1}$ is orthogonal to all the columns of $\boldsymbol{H}^{T} \boldsymbol{H}$ except the $i$ th column. If a column of $\boldsymbol{H}^{T} \boldsymbol{H}$ is projected onto the rows of $\boldsymbol{H}^{T} \boldsymbol{H}^{-1}$ only one of the dot products will be non-zero. Therefore for each column of $\boldsymbol{H}^{T} \boldsymbol{H}$ there will be only one row in $\boldsymbol{H}^{T} \boldsymbol{H}^{-1}$ that is non-orthogonal and consequently only one non-zero term in each row of the resulting matrix. The geometrical representation of the decorator is shown in Fig. 3.8. Notice that $\boldsymbol{H}^{T} \boldsymbol{s}_{\boldsymbol{1}}$ is the $1^{\text {st }}$ column of $\boldsymbol{H}^{T} \boldsymbol{H}$ and is orthogonal to the $2^{\text {nd }}$ row of $\boldsymbol{H}^{T} \boldsymbol{H}^{-1}$. Similarly $\boldsymbol{H}^{T} \boldsymbol{s}_{2}$ is the $2^{\text {nd }}$ 


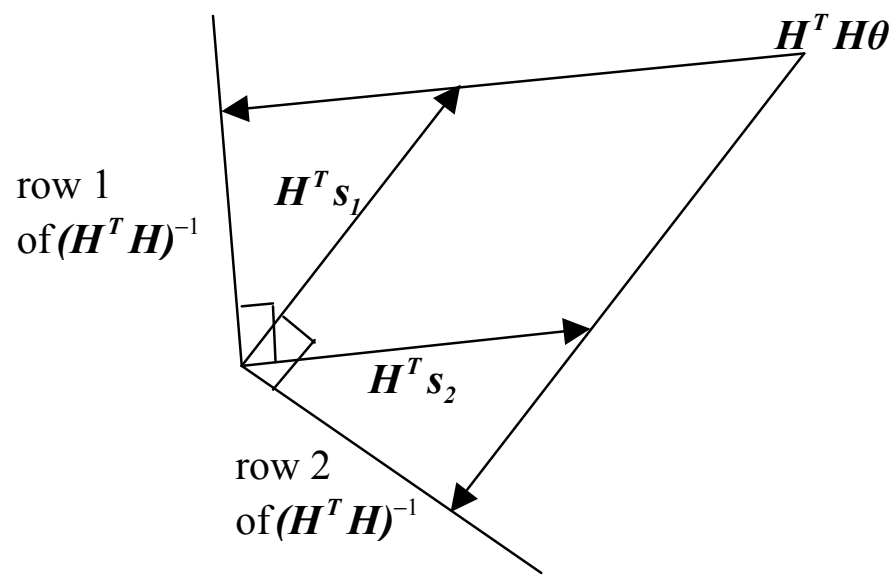

Fig. 3.8 Geometrical representation of a decorrelator for 2 users

column of $\boldsymbol{H}^{T} \boldsymbol{H}$ and is orthogonal to the $1^{\text {st }}$ row of $\boldsymbol{H}^{T} \boldsymbol{H}^{-1}$. Therefore the decorrelation operation projects the matched filter output of each user in a direction that is orthogonal to the matched filter outputs for all the other users. Since the projections are mutually non-orthogonal the noise is not white and its covariance is $\boldsymbol{H}^{T} \boldsymbol{H}^{-1}$. This usually results in a lower SNR than at the output of the matched filter [Jun96]. Lets consider the case when $K=2$ and the correlation between the two codes is $\rho=0.3$. Then the noise covariance at the output of the matched filter and the decorrelator detector is given as

$$
\boldsymbol{C}_{M \boldsymbol{F}}=\boldsymbol{H}^{\boldsymbol{T}} \boldsymbol{H}=\left[\begin{array}{cc}
1 & 0.3 \\
0.3 & 1
\end{array}\right] \quad \boldsymbol{C}_{\boldsymbol{D C}}=\left(\boldsymbol{H}^{\boldsymbol{T}} \boldsymbol{H}\right)^{-1}=\left[\begin{array}{cc}
1.10 & -0.33 \\
-0.33 & 1.10
\end{array}\right]
$$

It can be seen from (3.22) that the noise at the output of the decorrelator is enhanced by a factor of $1 /\left(1-\rho^{2}\right)$, i.e., the noise increases exponentially with increasing $\rho$. Therefore it is expected that the performance of the decorrelator will rapidly degrade with increasing $\rho$. 


\subsection{Simulation Results}

The performance of the MVU estimator is compared with the correlation receiver for MSK through Matlab simulations. Since we have used real notation throughout the chapter we used real passband signals for our simulations instead of using complex baseband signals. The carrier frequency was chosen to be $f_{c}=10 \mathrm{~Hz}$ and the sampling frequency to be $f_{s}=4 f_{c}$. The symbol period is normalized to one.

It is seen that the MVU estimator outperforms the correlation receiver (matched filter receiver) if the phase deviation from orthogonality is less than $50 \mathrm{deg}$. The performance of the MVU is then compared to the conventional receiver at a phase deviation of $10 \mathrm{deg}$. over a range of SNRs (Fig. 3.10). It is seen that the MVU provides a gain of about $2 \mathrm{~dB}$ for moderate to high SNRs. The performance of the MVU is then compared to the conventional receiver for various SIRs (Fig. 3.11) and it is found that the MVU still

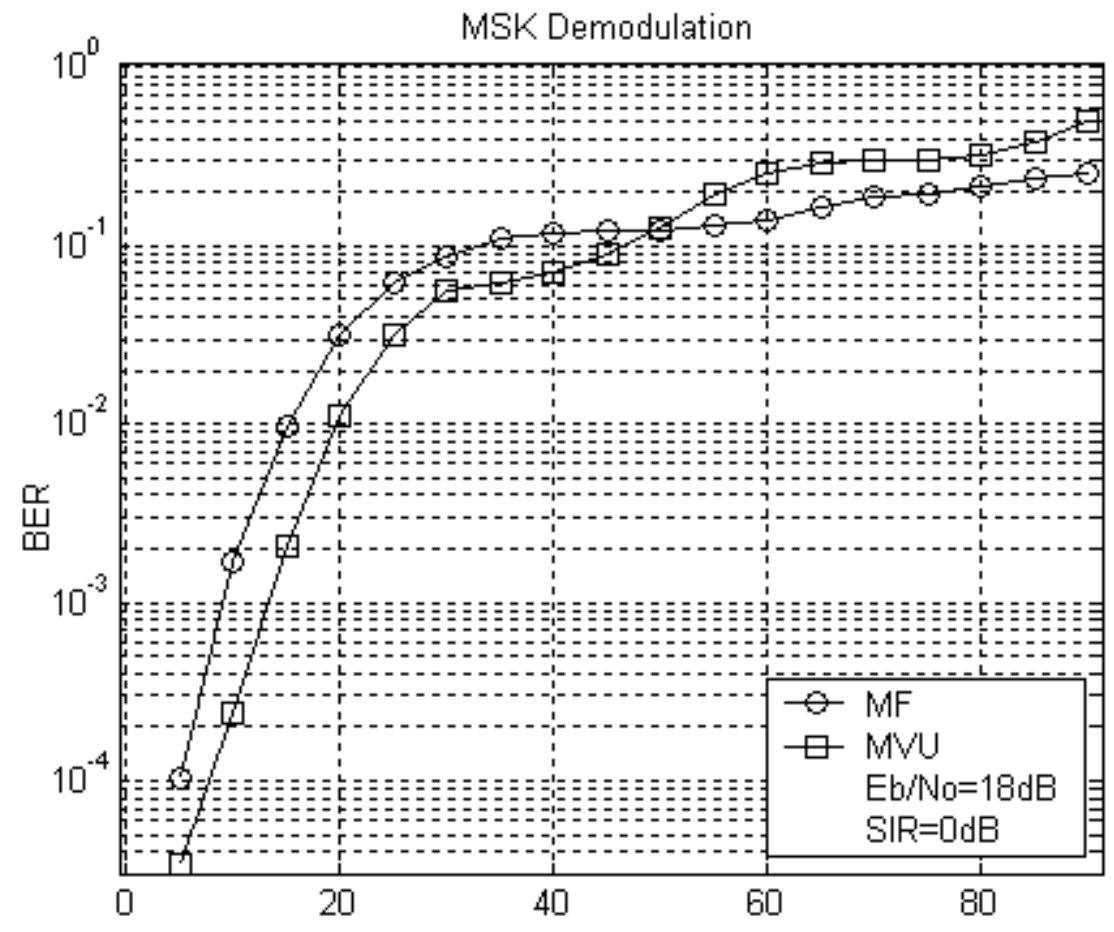

Phase deviation from orthogonality

Fig. 3.9 MSK bit error rate at $\mathrm{SIR}=0 \mathrm{~dB}$ and $\mathrm{Eb} / \mathrm{No}=18 \mathrm{~dB}$ 


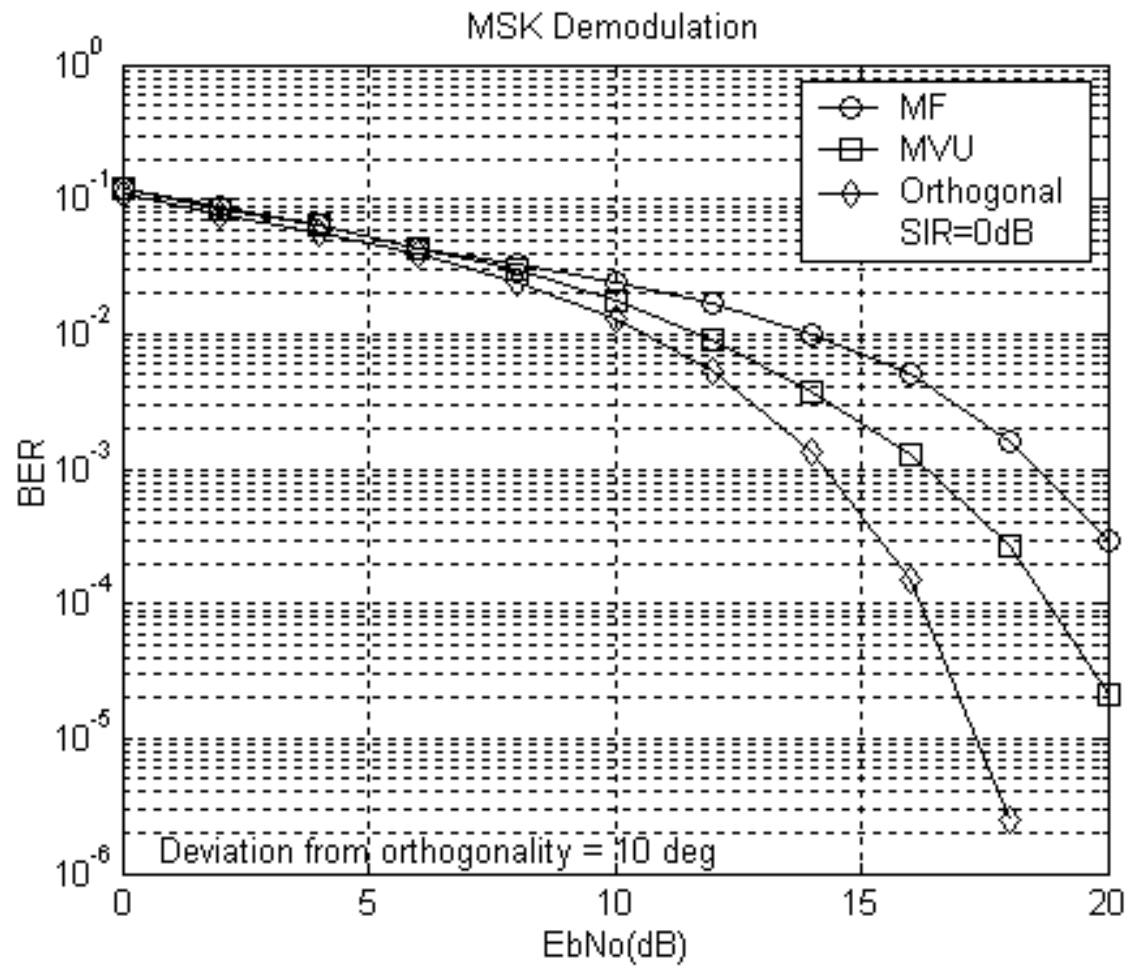

Fig. 3.10 MSK bit error rate at $\mathrm{SIR}=0 \mathrm{~dB}$ and phase deviation $=10 \mathrm{deg}$

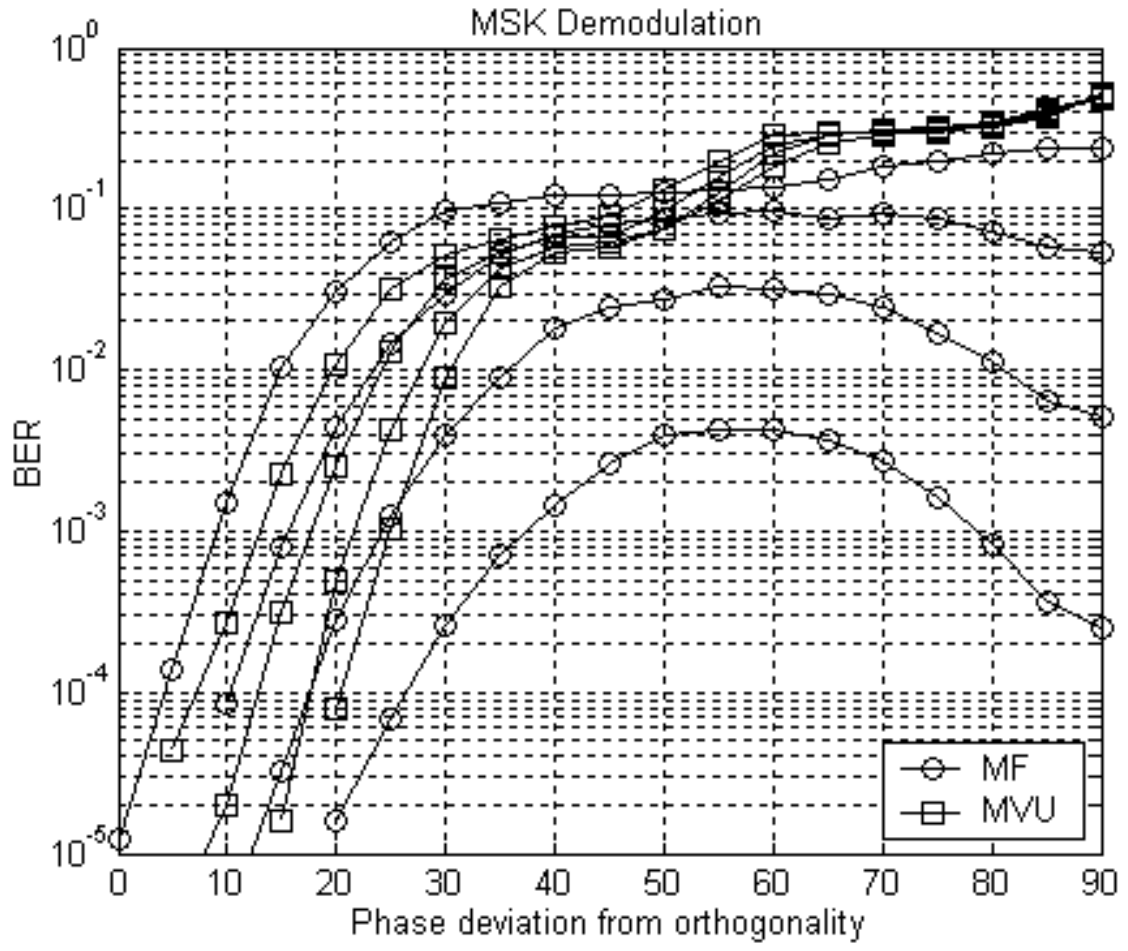

Fig 3.11 MSK bit error rate at $\mathrm{Eb} / \mathrm{No}=18 \mathrm{~dB}$ and $\mathrm{SIR}=0,1,2,3 \mathrm{~dB}$ 
performs slightly better for low phase deviations but is much worse at higher phase deviations. The performance of the minimum mean square error (MMSE) estimator was also compared to the conventional receiver. It was found that the performance of the MMSE estimator was similar to that of the MVU estimator. This is an expected result because we are considering low SIR, high SNR scenarios and for high SNRs the MMSE estimator converges to the MVU estimator.

Fig. 3.12 shows the error performance of the MF and MVU for various phase deviations and a frequency offset of 0.5. It is seen that the MVU still outperforms the MF in the low phase deviation region. However the difference between the 2 is now much larger. The performance of the MVU and the MF is then compared for a phase deviation of $30 \mathrm{deg}$. over a range SNRs (Fig. 3.13). It is seen that the MVU provides a gain of about $3 \mathrm{~dB}$ in the moderately high SNR region.

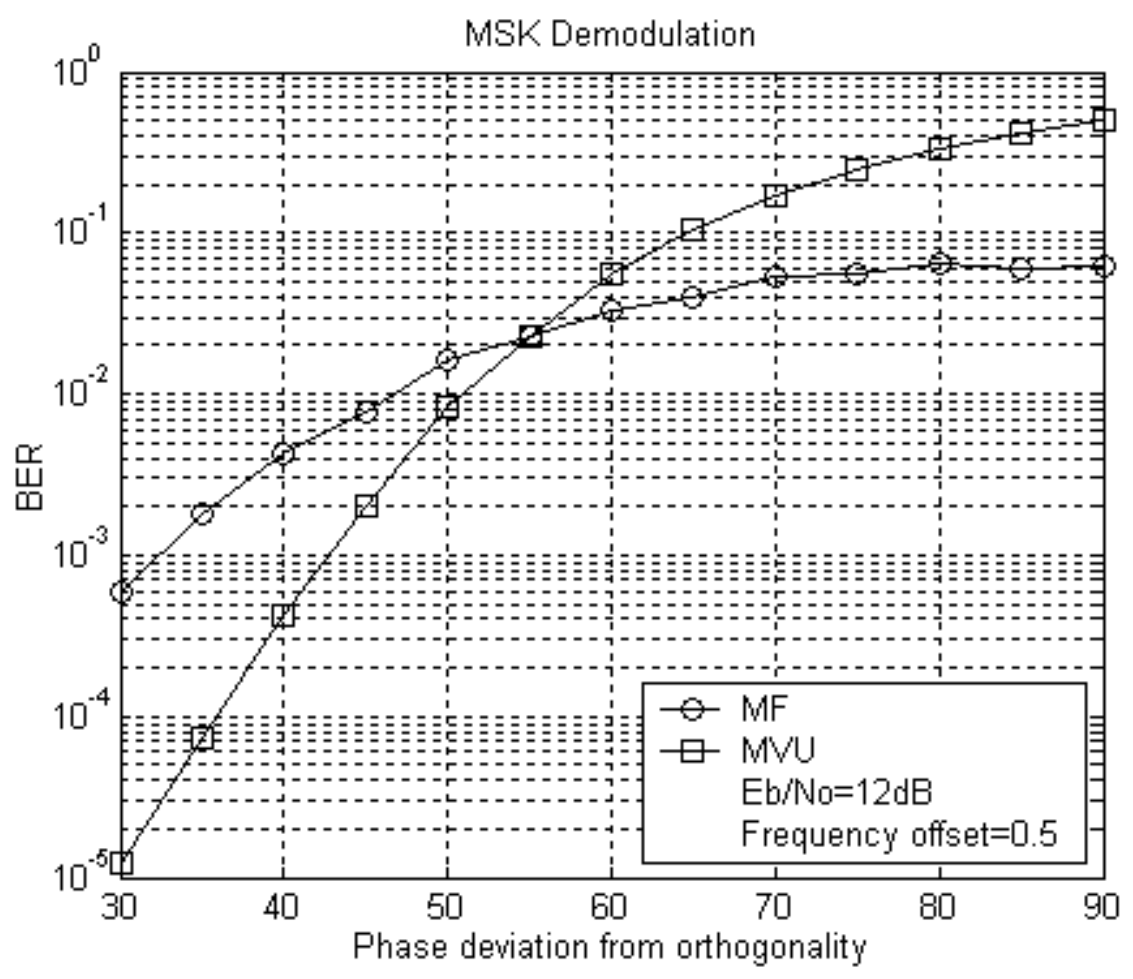

Fig. 3.12 MSK bit error rate at $\mathrm{SIR}=0 \mathrm{~dB}$ and $\mathrm{Eb} / \mathrm{No}=12 \mathrm{~dB}$ 


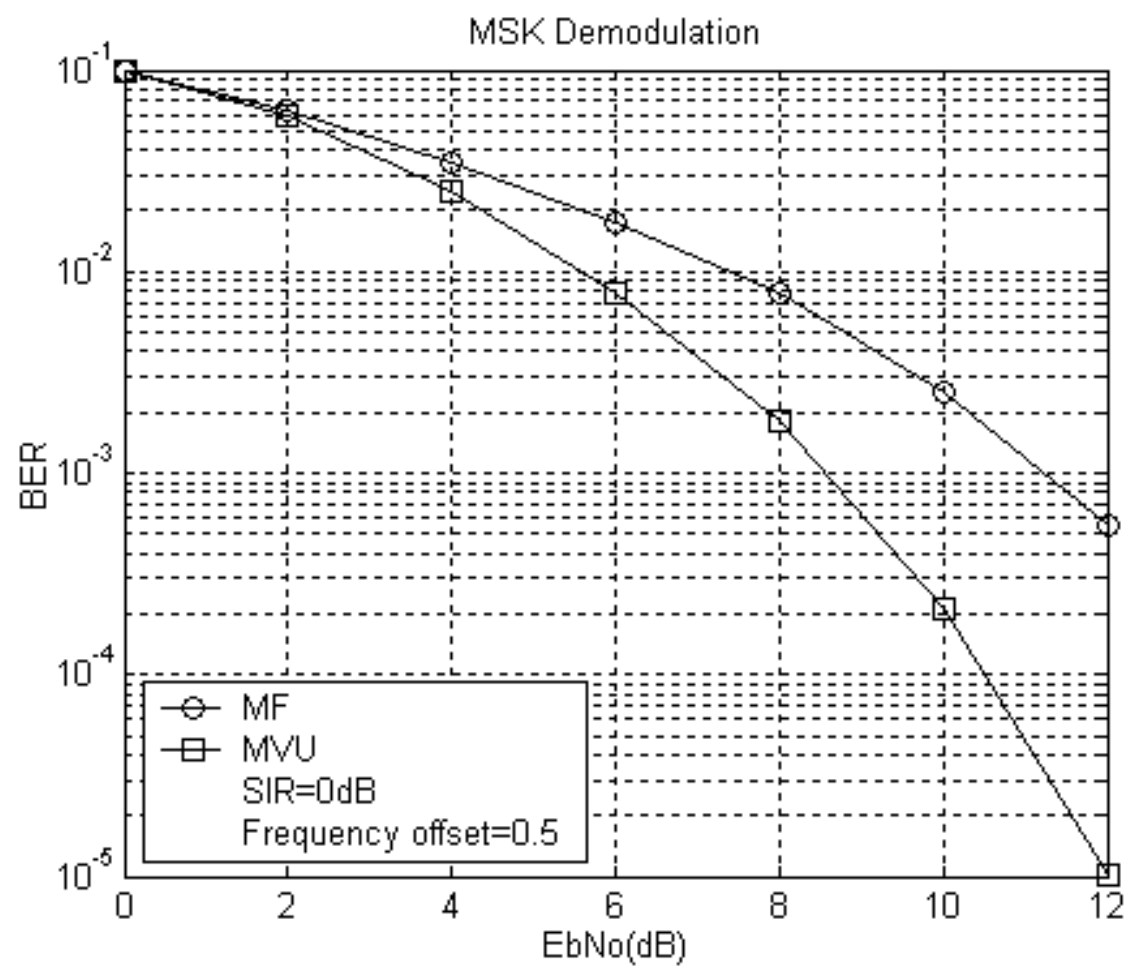

Fig. 3.13 MSK bit error rate for $\mathrm{SIR}=0 \mathrm{~dB}$, frequency offset $=0.5$ and phase offset $=30 \mathrm{deg}$

\subsection{Summary}

The concepts of Minimum Variance Unbiased Estimation (MVU) and Cramer-Rao Lower Bound are introduced. It is shown that MSK signals can be written in the linear model form, hence an MVU exists that satisfies the CRLB and the solution to the MVU problem results in the typical correlation receiver used in most communication systems. However if the basis functions are not orthogonal the exact solution needs to be calculated. The exact solution takes care of the correlation between the basis functions. It was shown in chapter 2 that with proper phase difference two MSK signals are completely orthogonal to each other, therefore, the problem of co-channel interference can be viewed as a problem of non-orthogonal basis functions. These concepts are then extended to wideband signals and it is shown that the decorrelator based multiuser detector is also the minimum variance unbiased estimator. The decorrelation operation is studied in more detail and a graphical interpretation is given. We have studied the 
decorrelation operation in the context of spread spectrum signals as there has been considerable research on this topic, however, these concepts can be extended to many problems in communications where the basis functions are non-orthogonal.

Simulation results have shown that the MVU estimator provides significant gains over the typical correlation receiver if the phase deviation between the two signals is less than $30 \mathrm{deg}$. However it fails if the phase deviation is very high. Since we are considering the $2^{\text {nd }}$ MSK signal to be an interferer it is not guaranteed that the two signals will be orthogonal or the phase deviation would be small. Therefore there will be no advantage of using an MVU in such an environment. However if both the signals originate from the same system and if we have some control over the phase difference between them then we can expect to have some advantage in using an MVU. 


\section{Chapter IV Autoregressive Parameter Estimation}

\subsection{Introduction}

In the previous chapter we had developed the estimation problem in communications for the case when the signal model is known and can be represented in linear form. However, in practice, this may not always be the case. Most communication systems use some form of pulse shaping to restrict the spectral leakage into adjacent channels and it is difficult if not impossible to represent the pulse shaped signals in linear form. In some cases we might not even know the type of frequency modulation and would like to have a general receiver architecture that can demodulate any frequency modulated signal. This has many applications in military for surveillance and reconnaissance.

It is also possible that the exact carrier frequency is unknown or carrier phase reference is not available. The former is true if the transmitter oscillator circuit is inaccurate or if the signal is an interferer from another system. The latter is the case if the phase synchronization circuitry is not implemented at the receiver. Furthermore, coherent reception techniques require accurate channel estimation either through training sequences or by using some blind channel estimation techniques.

In this chapter we will look at the problem of demodulating frequency modulated signals in time-varying fading environments as a problem of frequency estimation for short data records. We will focus on autoregressive modeling approach that has been widely used for speech processing and high-resolution spectral estimation. It has been shown in [We196] that AR modeling techniques can be used for demodulation of AMPS/NAMPS signals and we would extend this work to GMSK signal that is being widely used in many wireless communication systems. 


\subsection{Instantaneous Frequency}

The concept of instantaneous frequency (IF) is encountered in many fields where the signal of interest has a frequency that is time dependant. These signals are referred to as nonstationary signals. Any stationary signal can be represented as a sum of sine and cosine waves with particular frequencies. However for nonstationary signals, e.g. a chirp, this does not make sense because the signal sweeps over a band of frequencies during the observation interval. The usual approach to this problem is to make the observation interval so small that the signal can be assumed to be a sine wave (assuming that signal is monocomponent). This means that data record available for the IF estimation would be very small and this leads to the problem of frequency resolution that is discussed in the following sections.

If the signal of interest can be written in the form

$$
s(t)=a \cos (\phi(t))=a \cos \left[\int_{0}^{\tau} 2 \pi f_{i}(t) d t+\theta\right]
$$

where $a$ is the amplitude and $\phi(t)$ is the phase, then the instantaneous frequency of the signal, $f_{i}(t)$, can then be expressed as

$$
f_{i}(t)=\frac{1}{2 \pi} \frac{d \phi(t)}{d t}
$$

i.e. the instantaneous frequency is the time derivative of the phase [Bos92.1]. Notice that if the frequency, $f_{i}(t)$, is constant (4.1) is reduced to

$$
s(t)=a \cos [2 \pi f t+\theta]
$$

If the amplitude, $a$, or phase, $\theta$, is made time varying we have amplitude modulation and phase modulation respectively. Usually the IF of communication signals is composed of a 
constant frequency component called the carrier and a time varying component that contains the information. Mathematically

$$
s(t)=a \cos (\phi(t))=a \cos \left[2 \pi f_{c} t+2 \pi \int_{0}^{\tau} f_{m}(t) d t+\theta\right]
$$

and the instantaneous frequency is

$$
f_{i}(t)=f_{c}+f_{m}(t)
$$

where $f_{c}$ is the constant carrier component and $f_{m}$ is the information bearing component.

\subsection{Frequency Estimation Techniques}

A thorough comparison of the different spectrum estimation techniques can be found in [Kay81]. It gives a detailed explanation of each technique followed by a comparison of the bias and variance of these techniques for a 64-point sample sequence. The algorithmic procedure and computational complexity of each technique is also given. However it does not discuss some of the recent developments in subspace methods including MUSIC and ESPRIT. A detailed discussion about these techniques can be found in [Kay88], [Roy89] and [The92]. A comprehensive discussion on the IF estimation techniques can also be found in [Bos92.1] and [Bos92.2]. [Bos92.1] gives a historical perspective on the IF estimation problem and lays down the mathematical framework. [Bos92.2] presents the different IF estimation techniques available and compares them in terms of MSE. The author gives particular emphasis to IF estimation techniques based on time-frequency distributions and compares them to other traditional techniques.

To have a better understanding of the different frequency estimation techniques and their relationships we divide them into 3 categories. 
1. Classical techniques based on Fourier transform

2. Parametric techniques based on rational transfer function models

3. Subspace methods

The latter two categories are sometimes grouped together into what are called the modelbased techniques.

\subsubsection{Classical Techniques}

The Fourier transform has been known as a spectrum estimator for around 200 years and was first used by Schuster for determining the periodicities in the sun-spot numbers. Wiener and Khinchin, working separately, developed the relationship between the Fourier transform of the autocorrelation sequence of a random process and its power spectral density. The first efficient algorithm for determining the power spectral density (PSD) of a sampled data sequence using the autocorrelation approach was proposed by Blackman and Tukey in 1958. In 1965 Cooley and Tukey proposed the FFT algorithm that operates directly on the data. The resulting spectrum is called the periodogram and it continues to be the most popular method for determining the spectrum of a sampled data sequence.

There are two major drawbacks of these techniques. The first is that the resolution available with these techniques is very limited and is approximately equal to the reciprocal of the observation interval. Mathematically

$$
\Delta f=\frac{F_{s}}{N}
$$

or

$$
\Delta f=\frac{\text { number of samples / observation interval }}{\text { number of samples }}
$$


or

$$
\Delta f=\frac{1}{\text { observation interval }}
$$

where $F_{s}$ is the sampling frequency and $N$ is the number of samples. Now let's suppose that we want to estimate the frequency of a digital FM signal over a symbol period and the number of samples used for the estimate, $N$, is equal to the number of samples per symbol. Therefore we have

$$
\Delta f=\frac{F s}{N}=\frac{F s}{F s / R_{s}}=R_{s}
$$

It can be seen that the frequency resolution provided by the classical methods is equal to the symbol rate $R_{s}$. In the context of co-channel interference rejection this seems be insufficient because the difference in carrier frequencies is usually a fraction of the symbol rate. The Fourier transform can also be considered a non-coherent demodulation scheme and the frequency resolution it provides is the same as the minimum spacing required for non-coherent detection of FSK signals [Sk188]. It must also be noted that the frequency resolution cannot be improved by simply increasing the sampling rate.

The second problem is side-lobe leakage that distorts the spectral estimate and may also mask some lower power signals in adjacent bins. The problems of frequency resolution and side-lobe leakage can be traced back to the windowing operation performed on the data. By taking a finite length sequence we are assuming that the data outside the window is zero. This is not a very good assumption as we have some information about statistical properties of the unobserved data and therefore can come up with better models. This is the prime motivation for rational transfer function modeling. Sometimes non-rectangular windows are used to reduce the side-lobe leakage in adjacent bins but this is at the expense of a wider main lobe. 


\subsubsection{Rational Transfer Function Modeling}

Rational transfer functions have been used extensively for modeling many types of discrete time signals e.g. speech signals. An autoregressive moving average (ARMA) model is the most general rational transfer function model and can be represented by the following difference equation

$$
x[n]=-\sum_{k=1}^{p} a[k] x[n-k]+\sum_{k=0}^{q} b[k] u[n-k]
$$

where $u[\mathrm{n}]$ is the input sequence also called the driving noise sequence with variance $\sigma^{2}$ and $x[\mathrm{n}]$ is the output sequence. It is seen that at any instant the output is dependant on $(q+1)$ inputs (MA) as well as $p$ previous outputs (AR). The coefficients $a[\mathrm{k}]$ and $b[\mathrm{k}]$ and the driving noise $u[\mathrm{n}]$ completely define the ARMA model. The transfer function of an ARMA model can be written as

$$
H(z)=\frac{B(z)}{A(z)}
$$

where

$$
\begin{aligned}
& A(z)=\sum_{k=0}^{p} a[k] z^{-k} \\
& B(z)=\sum_{k=0}^{q} b[k] z^{-k}
\end{aligned}
$$

and the power spectral density (PSD) of the output sequence is

$$
P_{x x}(f)=\sigma^{2}\left|\frac{B(f)}{A(f)}\right|^{2}
$$


Note that $u[\mathrm{n}]$ is an inherent part of the model and should not be confused with the observation noise. The ARMA model is also known as the pole-zero model. If we make all the denominator coefficients except $a[0]$ equal to zero we have the MA model. The difference equation given in (4.7) would then be reduced to

$$
x[n]=\sum_{k=0}^{q} b[k] u[n-k]
$$

and the corresponding PSD would be

$$
P_{x x}(f)=\sigma^{2}|B(f)|^{2}
$$

Similarly if we make all the numerator coefficients except $b[0]$ equal to zero we have the AR model.

$$
x[n]=-\sum_{k=1}^{p} a[k] x[n-k]+u[n]
$$

and the PSD of the AR process would be

$$
P_{x x}(f)=\frac{\sigma^{2}}{|A(f)|^{2}}
$$

It has been assumed that $a[0]=b[0]=1$ because any gain can be lumped with $\sigma^{2}$. The selection of any one of these models depends upon the PSD of the data we want to represent. If the data consists of pure sinusoids then the AR model is usually the best choice. This is because the poles of the AR model can accurately represent discrete components in the frequency domain. However if the PSD has nulls an MA model would be more appropriate. An MA model can also represent a process with spectral peaks however the model order required would be much higher. In general any of the three 
models can be used to represent a process, however, the model orders would be different and it is usually desirable to select the one with the lowest order. This results in a more accurate estimation of the model parameters from a short data record.

The estimation of the AR parameters is based on a known autocorrelation sequence $\left\{r_{x x}[0], r_{x x}[1], \ldots, r_{x x}[p]\right\}$. If it is assumed that the autocorrelation sequence outside this window is equal to zero the resulting spectrum would be smeared and coarse frequency resolution would be obtained. This would actually be the spectrum obtained by the Blackman Tukey (BT) method. The PSD obtained by the BT method can be written as

$$
\hat{P_{B T}}=\sum_{k=-(N-1)}^{N-1} r_{x x}[k] e^{-j 2 \pi f k}
$$

where $N$ is the number of samples. On the other hand the PSD for the AR process is

$$
\hat{P_{A R}}=\sum_{-\infty}^{\infty} \hat{r_{x x}}[k] e^{-j 2 \pi f k}
$$

where

$$
\hat{r}_{x x}[k]= \begin{cases}r_{x x}[k] & \text { for } 0 \leq \mathrm{k} \leq \mathrm{p} \\ -\sum_{l=1}^{p} \hat{a}[l] \hat{r_{x x}}[k-l] & \text { for } \mathrm{k}>\mathrm{p}\end{cases}
$$

It is seen that the autocorrelation sequence outside the window is extrapolated using the autocorrelation sequence for $(p+1)$ previous lags. Thus a reasonable estimate is made about the data outside the window rather than assuming it to be to zero as is done in the classical techniques. This is the main reason for the high resolution provided by the AR spectral estimator. 
A technique that is closely related to AR spectral estimation is the Maximum Entropy Spectral Estimation (MESE) proposed by Burg in 1975. The fundamental concept behind Maximum Entropy method is that the autocorrelation sequence outside the window can be obtained by selecting a time series that has the maximum entropy, i.e., it is the most random time series that matches the known autocorrelation sequence (for $p$ lags). Since the constraints on the time series are minimum it has the maximum randomness and the resulting spectral estimator has the minimum bias. It has been shown that for a Gaussian random process and known autocorrelation function the maximum entropy method and AR method yield the same solution [Kay88].

\subsubsection{Subspace Methods}

Subspace methods are model-based techniques for estimating discrete components of the spectrum. These techniques are based on the principle of separating the data into a signal subspace and a noise subspace. A Soviet scientist, V. F. Pisarenko, first introduced this concept in 1973 and his technique is known as Pisarenko harmonic decomposition. This concept was later used to develop more advanced techniques such as MUSIC (multiple signal classification) and ESPRIT (estimation of signal parameters via rotational invariance). The ESPRIT algorithm was originally proposed for high-resolution direction of arrival (DOA) estimation [Roy89] but is now widely used for high-resolution frequency estimation.

In subspace methods the data is assumed to consist of $M$ complex exponentials in white noise. Mathematically

$$
x[n]=\sum_{i=1}^{M} A_{i} s_{i}[n]+w[n] \quad n=0,1, \ldots N-1
$$

where

$$
S_{i}[n]=e^{j w_{i} n}
$$


and

$$
A_{i}=\left|A_{i}\right| e^{j \phi_{i}}
$$

As with any other model-based technique the performance of subspace methods depends on how closely the physical process matches this mathematical model. The fundamental concept behind these techniques is that eigenvectors of the correlation matrix $\boldsymbol{R}_{x x}$ corresponding to the $M$ largest eigenvalues represent the complex exponentials in the data. These eigenvectors define the signal subspace. The remaining eigenvalues are approximately equal to the noise variance and the corresponding eigenvectors form the noise subspace. The process of determining the frequency of the complex exponentials from the eigenvectors of the correlation matrix differentiates the different subspace techniques.

The Pisarenko harmonic decomposition (PHD) is rarely used in practice; however, it serves to illustrate the basic concepts of the subspace methods. In PHD it is assumed that the number of complex exponentials, $M$, is known and the correlation matrix has dimensions $(M+1) \times(M+1)$. Therefore the number of eigenvectors is one greater than the number of complex exponentials. The eigenvector corresponding to the smallest eigenvalue is the noise eigenvector and by definition it is orthogonal to the signal vectors. Mathematically

$$
\left.\boldsymbol{w}^{H} \boldsymbol{e}_{N}\right|_{w=w_{i}}=0 ; \quad i=1,2, \ldots, M
$$

where $\boldsymbol{e}_{N}$ is the noise eigenvector and 


$$
\boldsymbol{w}=\left[\begin{array}{c}
1 \\
e^{j w} \\
\vdots \\
e^{j(N-1) w}
\end{array}\right]
$$

represents the signal vector. The PHD pseudospectrum can then be written as

$$
\hat{P}\left(e^{j w}\right)=\frac{1}{\left|\boldsymbol{w}^{H} \boldsymbol{e}_{N}\right|^{2}}=\frac{1}{\boldsymbol{w}^{H} \boldsymbol{e}_{N} \boldsymbol{e}_{N}{ }^{H} \boldsymbol{w}}
$$

It is called the pseudospectrum because it only gives information about the location of the complex exponentials and not their power. The denominator of (4.21) goes to zero at the signal frequency $\boldsymbol{w}_{i}$ and this theoretically results in an infinite peak in the spectrum.

The MUSIC method removes the limitation on the maximum size of the correlation matrix. If the number of complex exponentials is $M$ then the correlation matrix can be of any size $N>M+1$. The number of noise eigenvectors is equal to $N-M$ as opposed to only one for PHD and the pseudospectrum is defined as

$$
\hat{P}\left(e^{j w}\right)=\frac{1}{\boldsymbol{w}^{H} \boldsymbol{E}_{\text {noise }} \boldsymbol{E}_{\text {noise }}^{H} \boldsymbol{w}}
$$

where $\boldsymbol{E}_{\text {noise }}$ is the matrix of noise eigenvectors defined as

$$
\boldsymbol{E}_{\text {noise }}=\left[\begin{array}{llll}
\boldsymbol{e}_{M+1} & \boldsymbol{e}_{M+2} & \cdots & \boldsymbol{e}_{N}
\end{array}\right]
$$

The term $\boldsymbol{E}_{\text {noise }} \boldsymbol{E}_{\text {noise }}^{H}$ is called the projection matrix for the noise subspace $\boldsymbol{P}_{\text {noise }}$ and (4.21) is usually expressed in terms of $\boldsymbol{P}_{\text {noise }}$. Since the noise subspace is more defined than in PHD the signal vector is more constrained and this results in a better frequency estimate. 
ESPRIT is a comparatively new subspace technique that provides frequency resolution comparable to MUSIC. The original ESPRIT algorithm was presented in terms of the correlation matrix of the data and it had severe numerical problems. The least square (LS) and total least square (TLS) versions that were proposed later solved these problems and are the preferred methods for implementation [Man00].

The ESPRIT algorithm is different from other subspace methods in that it estimates the frequency of the discrete components using the signal subspace rather than the noise subspace. The key to this method is to break up the signal subspace into two staggered subspaces. Lets define a signal matrix $\boldsymbol{S}$ such that

$$
\boldsymbol{S}=\left[\begin{array}{cccc}
\vdots & \vdots & & \vdots \\
s_{1}[n] & s_{2}[n] & \cdots & s_{M}[n] \\
\vdots & \vdots & & \vdots
\end{array}\right]
$$

or

$$
\boldsymbol{S}=\left[\begin{array}{cccc}
1 & 1 & \cdots & 1 \\
e^{j \omega_{1}} & e^{j \omega_{2}} & \cdots & e^{j \omega_{M}} \\
\vdots & \vdots & \ddots & \vdots \\
e^{j \omega_{1}(N-1)} & e^{j \omega_{2}(N-1)} & \cdots & e^{j \omega_{M}(N-1)}
\end{array}\right]
$$

then two matrices $S_{1}$ and $S_{2}$ are formed by eliminating the last and first row of $S$ respectively.

$$
\boldsymbol{S}=\left[\begin{array}{c}
\boldsymbol{S}_{1} \\
* * *
\end{array}\right]=\left[\begin{array}{c}
* * * \\
\boldsymbol{S}_{2}
\end{array}\right]
$$

These two matrices are related as

$$
S_{2}=S_{1} \Phi
$$

where 


$$
\boldsymbol{\Phi}=\left[\begin{array}{cccc}
e^{j \omega_{1}} & 0 & \cdots & 0 \\
0 & e^{j \omega_{2}} & \cdots & 0 \\
\vdots & \vdots & \ddots & \vdots \\
0 & \cdots & 0 & e^{j \omega_{M}}
\end{array}\right]
$$

It is obvious that the matrix $\boldsymbol{\Phi}$ contains the frequency information. If $\boldsymbol{S}_{1}$ and $\boldsymbol{S}_{2}$ were known then $\boldsymbol{\Phi}$ could have been easily determined using a least squares approach. However we can estimate the signal subspace by performing singular value decomposition on the data matrix $\boldsymbol{X}=\boldsymbol{U} \boldsymbol{\Sigma} \boldsymbol{V}^{\boldsymbol{H}}$. The columns of $\boldsymbol{V}$ (eigenvectors of $\boldsymbol{X}$ ) corresponding to the $M$ largest singular values of $\Sigma$ form the signal subspace. Let $\boldsymbol{V}_{s}$ be the matrix formed by the signal eigenvectors and $V_{1}$ and $V_{2}$ be the matrices formed by eliminating the last and first rows of $\boldsymbol{V}_{s}$ respectively. Then

$$
V_{2}=V_{1} \Psi
$$

and $\boldsymbol{S}$ and $\boldsymbol{V}_{s}$ are related through a linear transformation $\boldsymbol{T}$ such that

$$
S_{1}=V_{1} T \quad S_{2}=V_{2} T
$$

Now from (4.24) we have

$$
S_{2}=S_{1} \Phi
$$

or

$$
V_{2} T=V_{1} T \Phi
$$

or

$$
V_{1} \Psi T=V_{1} T \Phi
$$

or

$$
\Psi T=T \Phi
$$

It can be easily recognized that $\boldsymbol{T}$ and $\boldsymbol{\Phi}$ are the eigenvectors and eigenvalues of $\boldsymbol{\Psi}$ respectively. Therefore the problem of determining $\boldsymbol{\Phi}$ is reduced to finding the matrix of eigenvalues of $\boldsymbol{\Psi}$. Here $\boldsymbol{\Psi}$ can be found as the least squares solution to (4.25).

$$
\boldsymbol{\Psi}=\left(V_{1}^{H} V_{1}\right)^{-1} V_{1}^{H} V_{2}
$$

A discussion about total least squares ESPRIT can be found in [The92] and [Man00]. 


\subsection{Model Selection}

Model based spectral estimation is a three-step procedure as shown in Fig. 4.1. The first step is to select a model based upon the a priori information about the data. We know that the signal is a non-stationary FM signal i.e. the frequency of the signal is time varying, however, over a small observation window the signal can be considered to be

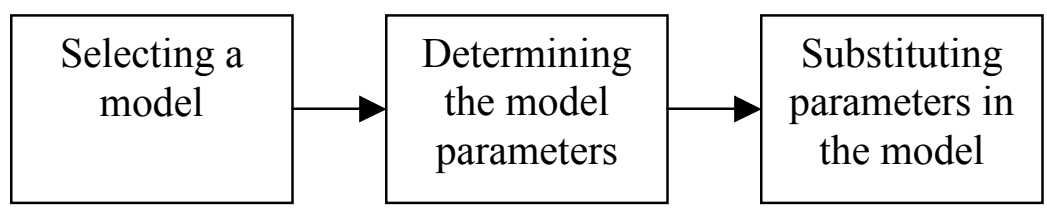

Fig 4.1 Steps involved in Model-based spectral estimation

stationary. Thus the signal essentially consists of a sinusoid in noise. In presence of cochannel interference there will be more than one sinusoidal component. Therefore the selected model should be able to represent sinusoidal components accurately and should also have a high frequency resolution. It should also be noted that even for a very small observation window that the frequency of the signal might not be constant therefore the model should also be able to represent narrow band processes.

In view of the above requirements it is obvious that the AR model would be the most appropriate. The classical techniques fail to provide high frequency resolution and the subspace methods fail to model narrowband processes [The92]. An MA or ARMA model may also be used however the required order for the MA model would be much higher than the AR model. The MA and ARMA models require solving a set of non-linear equations and are not as well understood as the AR model that has been widely used in many signal-processing applications. It has been shown in [We196] that AR model can be used to accurately represent AMPS/NAMPS signals and provides significant performance gains over the conventional Quadrature demodulator. Another motivation for selecting the AR model is that we can make use of sequential techniques that are 
computationally much more efficient then batch processing techniques and this approach has a close resemblance with traditional non-coherent demodulation techniques.

Now that we have selected a model for data the next step is to determine the model parameters. Another problem that needs attention is the model order selection. It has been reported in [We196] that a model order of $L=3 N / 4$ is optimum and this is what will be used in most of our simulations. Once the model parameters have been determined these are then substituted into the model to obtain the spectral estimate.

\subsection{Autoregressive Parameter Estimation}

The AR model given by (4.12) and (4.13) can be represented by the IIR filter shown in fig. 4.2. Here the driving noise sequence $u[\mathrm{n}]$ and the parameters $a_{1}, a_{2}, \ldots, a_{p}$ completely define the model.

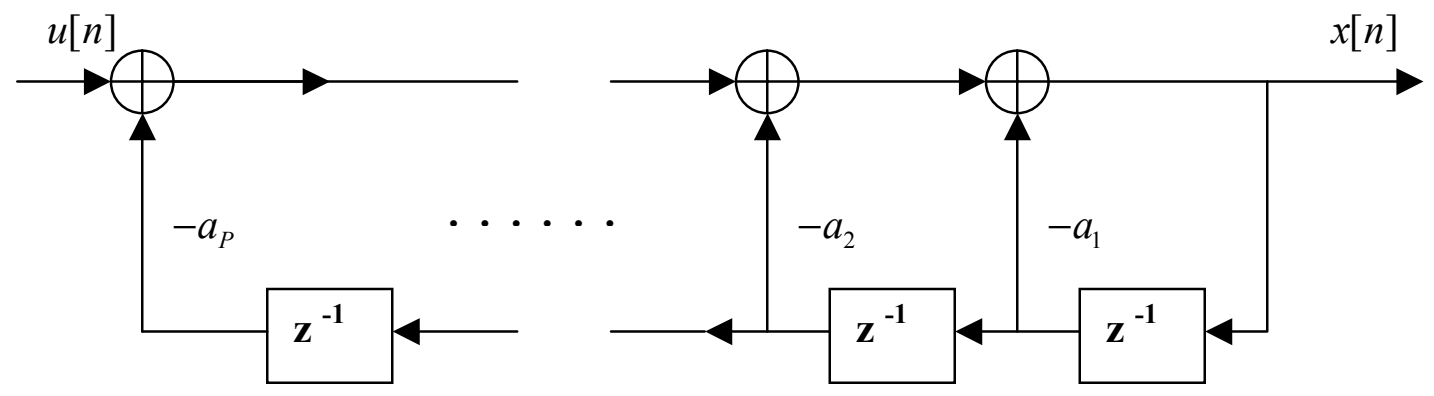

Fig. 4.2 Signal flow graph for the AR model

Several approaches have been proposed for determining the AR parameters. Most of the techniques are based on the assumption that the second moment statistics of the random process are known. However in practice this is seldom the case and the second moment statistics have to be estimated from the data. Thus the efficiency of these techniques is reduced. This prompted research into techniques that are directly applicable on the data and one method that has become quite popular is that of least squares. In the method of 
least squares the criterian for measuring the dissimiliarity between the data and the model is the sum of the errors rather than the traditional mean square error.

We will start with the maximum likelihood approach as it gives us some ideas about the statistical properties of the estimator and then extend the discussion to other approaches that are more important from a practical point of view.

\subsubsection{Maximum Likelihood Estimation}

In chapter 3 we had discussed minimum variance unbiased (MVU) estimation in detail. However for many practical problems the MVU estimator does not exist or even if it exists it cannot be found. In this case the most suitable approach is to find a maximum likelihood estimator (MLE). The MLE is asymptotically efficient, i.e., for large data records its performance approaches the Cramer-Rao lower bound and it can be found by following a definite set or rules. Furthermore if an efficient estimator does exist the maximum likelihood procedure will produce it [Kay93]. Therefore it is the most popular approach for practical estimation problems.

It has been shown in [Kay81] that the approximate maximum likelihood estimate for the AR parameters can be obtained by solving the Yule Walker equations.

$$
\left[\begin{array}{cccc}
r_{x x}[0] & r_{x x}[-1] & \cdots & r_{x x}[-(p-1)] \\
r_{x x}[1] & r_{x x}[0] & \cdots & r_{x x}[-(p-2)] \\
\vdots & \vdots & \ddots & \vdots \\
r_{x x}[p-1] & r_{x x}[p-2] & \cdots & r_{x x}[0]
\end{array}\right]\left[\begin{array}{c}
\hat{a}[1] \\
\hat{a}[2] \\
\vdots \\
\hat{a}[p]
\end{array}\right]=-\left[\begin{array}{c}
r_{x x}[1] \\
r_{x x}[2] \\
\vdots \\
r_{x x}[p]
\end{array}\right]
$$

where $r_{x x}[k]$ is the autocorrelation function defined as

$$
r_{x x}[k]=E\left\{x^{*}[n] x[n+k]\right\}
$$


The Yule-Walker equations for the AR model or the Normal equations for a linear predictor can be derived from the orthogonality principle which states that the mean square error is minimized if the prediction error is made orthogonal to the observations [The92]. Mathematically

$$
E\left\{x[n-i] e^{*}[n]\right\}=0, \quad i=1,2, \ldots, p
$$

where $e[\mathrm{n}]$ is the prediction error in estimating $x[\mathrm{n}]$ from its $p$ previous values. The YuleWalker equations can be solved recursively using the Levinson algorithm [Kay93].

[Kay93] also derives the approximate CRLB for real AR parameters given as

$$
\operatorname{var}(\hat{a}[k]) \geq \frac{\sigma_{u}^{2}}{N}\left[R_{x x}^{-1}\right]_{k k} \quad k=1,2, \ldots, p
$$

where $\left[\boldsymbol{R}_{x x}\right]_{i j}=r_{x x}[i-j]$ is a $p \times p$ Toeplitz autocorrelation matrix. It is difficult to find the exact CRLB for AR parameters and details about this can be found in [Box70] and [Por87]. Let us now look at a special case when the order of the AR model is one, i.e., $p=1$.

$$
\operatorname{var} \hat{a}[1]) \geq \frac{\sigma_{u}^{2}}{N}\left[R_{x x}^{-1}\right]_{11}=\frac{\sigma_{u}^{2}}{N r_{x x}[0]}
$$

but

$$
r_{x x}[0]=\frac{\sigma_{u}^{2}}{1-a^{2}[1]}
$$

therefore 


$$
\operatorname{var}(\hat{a}[1]) \geq \frac{1}{N}\left(1-a^{2}[1]\right)
$$

It is obvious from the above expression that variance of the AR parameter would decrease as the value of $a[1]$ approaches unity therefore the model can accurately represent peaks in the spectrum. Secondly the variance also decreases as the number of samples $N$ increases. Let us now calculate the CRLB for the PSD of the AR(1) model using the rules for the transformation of parameters. The CRLB for the PSD can be calculated as

$$
\operatorname{var}\left(\hat{P_{x x}}(f)\right) \geq \frac{\left(\frac{\partial g}{\partial a[1]}\right)^{2}}{-E\left[\frac{\partial^{2} \ln p(x ; a[1])}{\partial^{2} a[1]}\right]}
$$

or

$$
\operatorname{var}\left(\hat{P}_{x x}(f)\right) \geq\left(\frac{\partial g}{\partial a[1]}\right)^{2} \operatorname{var}(\hat{a}[1])_{C R L B}
$$

where

$$
P_{x x}(f)=g(a[1])=\frac{\sigma^{2}}{\left|1+a[1] e^{-j 2 \pi f}\right|^{2}}
$$

Taking the derivative of (4.32) with respect to $a[1]$ and substituting the result in (4.31) gives the CRLB for the PSD as

$$
\operatorname{var}\left(P_{x x}(f)\right) \geq \frac{\sigma_{u}^{4}\left(1-a^{2}[1]\right)(\cos 2 \pi f+a[1])^{2}}{4 N\left(\frac{1+a^{2}[1]}{2}+a[1] \cos (2 \pi f)\right)^{4}}
$$


The CRLB for the PSD is now plotted for $\sigma_{u}=1, a[1]=-0.9$ and $N=100$. The plot is shown in fig. 4.2. It is seen that the CRLB is frequency dependant and the bound is much higher for frequencies near DC and at normalized frequency of 1 . This is understandable because near DC a slight change in frequency does not alter the signal much and at higher frequencies the number of samples is not sufficient to provide an accurate estimate. A detailed proof of (4.33) can be found in the Appendix A.

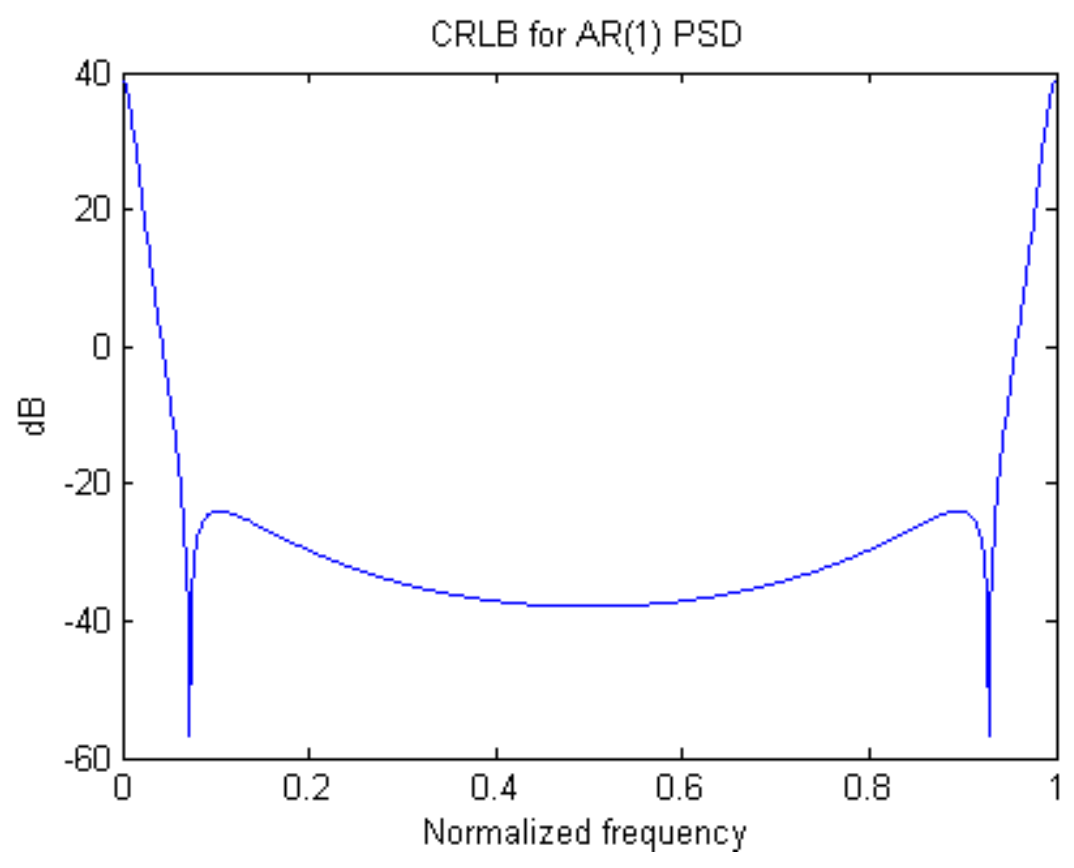

Fig. 4.3 Cramer-Rao lower bound for AR(1) PSD

\subsubsection{Least Squares Approach}

In the above discussion we have assumed that the autocorrelation function is either known or is estimated from the data. However in practice the autocorrelation function is not known and its estimate may be inaccurate, especially for short data records. Therefore it is desirable to come up with techniques that directly work on the data. The most popular approach in this regard is that of least squares.

In the general least squares approach it is assumed that the data is already known and the objective is to reduce the sum of squared error between the estimated sequence and the 
known sequence. The approach is purely deterministic and no assumptions are made about the statistical properties of the data. The least squares approach can be extended to the problem of determining the coefficients of a linear predictor or equivalently the parameters of the AR model. The linear prediction equation can be written as

$$
\hat{x}[n]=-a_{1} x[n-1]-a_{2} x[n-2]-\cdots-a_{p} x[n-p]
$$

where $\left\{a_{1}, a_{2}, \ldots, a_{p}\right\}$ are the prediction coefficients and the prediction error is

$$
e[n]=x[n]-\hat{x}[n]
$$

Let us now assume that $x[\mathrm{n}]$ is to predicted from sample $n=n_{I}$ to $n=n_{F}$. Then in matrix notation

$$
\left[\begin{array}{l}
\hat{x}\left[n_{I}\right] \\
\hat{x}\left[n_{I}+1\right] \\
\vdots \\
\hat{x}\left[n_{F}\right]
\end{array}\right]=-\left[\begin{array}{cccc}
x\left[n_{I}-1\right] & x\left[n_{I}-2\right] & \cdots & x\left[n_{I}-p\right] \\
x\left[n_{I}\right] & x\left[n_{I}-1\right] & \cdots & x\left[n_{I}-p+1\right] \\
\vdots & \vdots & & \vdots \\
x\left[n_{F}-1\right] & x\left[n_{F}-2\right] & \cdots & x\left[n_{F}-p\right]
\end{array}\right]\left[\begin{array}{l}
a_{1} \\
a_{2} \\
\vdots \\
a_{p}
\end{array}\right]
$$

where $p$ is the order of the prediction filter. This can be written in concise form as

$$
x=-X a
$$

It can be shown that the least squares solution to (4.37) is

$$
a=-X^{+} x
$$

where $\mathrm{X}^{+}$is known as the Moore-Penrose pseudoinverse and is defined as 


$$
\boldsymbol{X}^{+}=\left(\boldsymbol{X}^{H} \boldsymbol{X}\right)^{-1} \boldsymbol{X}^{H}
$$

therefore we have

$$
\boldsymbol{a}=-\left(\boldsymbol{X}^{H} \boldsymbol{X}\right)^{-1} \boldsymbol{X}^{H} \boldsymbol{x}
$$

Notice the similarity between the least squares solution for the model parameters as given in (4.40) and the minimum variance solution for the parameters of a linear model given in chapter 3. The main difference between the two is that in the latter an actual signal model is used whereas in the former the observation matrix $\boldsymbol{X}$ replaces the signal model.

The Moore-Penrose pseudoinverse given by (4.25) exists only if the matrix $\boldsymbol{X}$ is of full rank (this also ensures that $\boldsymbol{X}^{H} \boldsymbol{X}$ is of full rank). However if matrix $\boldsymbol{X}$ is rank deficient then we have to use some other methods for finding the inverse. One efficient method is using Singular Value Decomposition (SVD) which is given below.

Any $K$ x $P$ matrix $\boldsymbol{X}$ can be decomposed into a product of 3 matrices.

$$
\boldsymbol{X}=\boldsymbol{U} \boldsymbol{\Sigma} \boldsymbol{V}^{* T}
$$

where $\boldsymbol{U}$ is the $K \times K$ unitary matrix of left singular vectors, $V$ is the $P$ x $P$ unitary matrix of right singular vectors and $\Sigma$ is the $K \times P$ matrix of nonnegative real singular values.

$$
\boldsymbol{U}=\left[\begin{array}{cccc}
\mid & \mid & & \mid \\
u_{1} & u_{2} & \ldots & u_{K} \\
\mid & \mid & & \mid
\end{array}\right] \quad V=\left[\begin{array}{cccc}
\mid & \mid & & \mid \\
v_{1} & v_{2} & \ldots & v_{P} \\
\mid & \mid & & \mid
\end{array}\right] \quad \Sigma=\left[\begin{array}{cc}
S_{1} & 0 \\
0 & 0
\end{array}\right]
$$

where $S_{1}$ is a diagonal matrix of the nonzero singular values 


$$
\boldsymbol{S}_{1}=\left[\begin{array}{cccc}
\sigma_{1} & 0 & \cdots & 0 \\
0 & \sigma_{2} & \cdots & 0 \\
\vdots & \vdots & \ddots & \vdots \\
0 & 0 & \cdots & \sigma_{r}
\end{array}\right]
$$

and $r$ is the rank of $\boldsymbol{X}$. The Moore-Penrose pseudoinverse of $\boldsymbol{X}$ is defined as

$$
\boldsymbol{X}^{+}=\boldsymbol{V} \boldsymbol{\Sigma}^{+} \boldsymbol{U}^{* T}
$$

or

$$
\boldsymbol{X}^{+}=\sum_{k=1}^{r} \frac{1}{\sigma_{k}} \boldsymbol{v}_{k} \boldsymbol{u}_{k}^{{ }^{*} T}
$$

Where $\Sigma^{+}$is the pseudoinverse of $\Sigma$ and is defined as.

$$
\Sigma^{+}=\left[\begin{array}{cc}
S^{-1} & 0 \\
0 & 0
\end{array}\right]
$$

Even if $\boldsymbol{X}$ is of full rank the SVD is the preferred method for finding the inverse as it has much better computational properties. An AR frequency estimation technique that finds the inverse of $\boldsymbol{X}$ using (4.43) with $r=1$ is known as the Principal Component Method. A detailed discussion about the Principal Component Method and its noise rejection capabilities can be found in [Wel96].

\subsubsection{Sequential Approach}

The AR parameter estimation techniques discussed in the previous sections can be categorized as batch processing techniques where the parameters are recalculated each time and no information about their previous values is used. Since we know that the instantaneous frequency of the signal is gradually changing with time we can use a 
recursive approach for updating the $\mathrm{AR}$ parameters rather than recalculating them. This offers great computational savings

The AR parameters can be tracked using a gradient descent approach such as the least mean square (LMS) algorithm. The LMS algorithm is a 3-step procedure where the next data sample is predicted from its $p$ previous values, the prediction error is calculated, and the coefficients are updated. If $\boldsymbol{x}_{n}$ is the vector of data samples at time $\mathrm{n}$ and $\boldsymbol{a}_{\boldsymbol{n}}$ is the corresponding vector of linear prediction coefficients then the LMS algorithm can be written as [Bos92.2]

$$
\begin{aligned}
& \hat{x}[n+1]=\boldsymbol{a}_{n} \boldsymbol{x}_{n} \\
& e[n+1]=x[n+1]-\hat{x}[n+1] \\
& \boldsymbol{a}_{n+1}=\boldsymbol{a}_{n}+\mu e[n+1] \boldsymbol{x}_{n}{ }^{*}
\end{aligned}
$$

Where $\mu$ is a constant and it controls the rate of adaptation. A higher value of $\mu$ usually results in faster convergence but a higher residual error. If a higher convergence rate is required then the RLS algorithm might be used but this is at the cost of higher computational complexity. The RLS algorithm requires order $L^{2}$ computations as opposed to order $L$ for the LMS algorithm [Bos92.2] where $L$ is the length of the prediction filter.

\subsection{Frequency Component Determination}

As previously stated model based spectral estimation is a three-step procedure and the last step is to substitute the parameters in the model. Since the model parameters are the coefficients of an IIR filter with transfer function 


$$
H(z)=\frac{1}{1+a_{1} z^{-1}+a_{2} z^{-2}+\ldots+a_{m} z^{-p}}
$$

the spectral estimate can be written as

$$
\hat{P}(f)=\frac{\sigma^{2}}{\left|1+\sum_{k=1}^{p} \hat{a}[k] e^{-j 2 \pi f k}\right|^{2}}
$$

where $\sigma^{2}$ is the power of the driving noise sequence. If we need to estimate the frequency of a single sinusoid then it can be found from the peak of (4.46). Another method that is usually easier than finding the peak in the spectrum (or peaks if there is more than one sinusoid) is by finding the poles of the filter. The poles of a filter represent the peaks in the spectrum and they can be found as the roots of the denominator polynomial in (4.46). The frequency of a single sinusoidal component can be found by determining the angle of the pole closest to the unit circle. The pole-zero plot for a fifth order filter is shown in fig. 4.4. The pole closest to the unit circle represents the signal pole and the rest are just noise poles. It is seen that as the noise power increases the variance of the poles about their mean positions increases. The outward displacement of the noise poles results in false peaks in the spectrum. It has been shown in [We196] that the Principal Component Method shifts the noise poles inward and distributes them uniformly around the unit circle.

If the signal consists of a single sinusoid we can also calculate the frequency by simply finding the angle of the complex conjugate of the first coefficient [Bos92.2].

$$
\hat{f}_{i}(n)=\frac{1}{2 \pi} \arg \left[a_{1}^{*}\right]
$$

This is computationally much more efficient than finding the peaks in (4.35) or finding the roots of the denominator polynomial. 

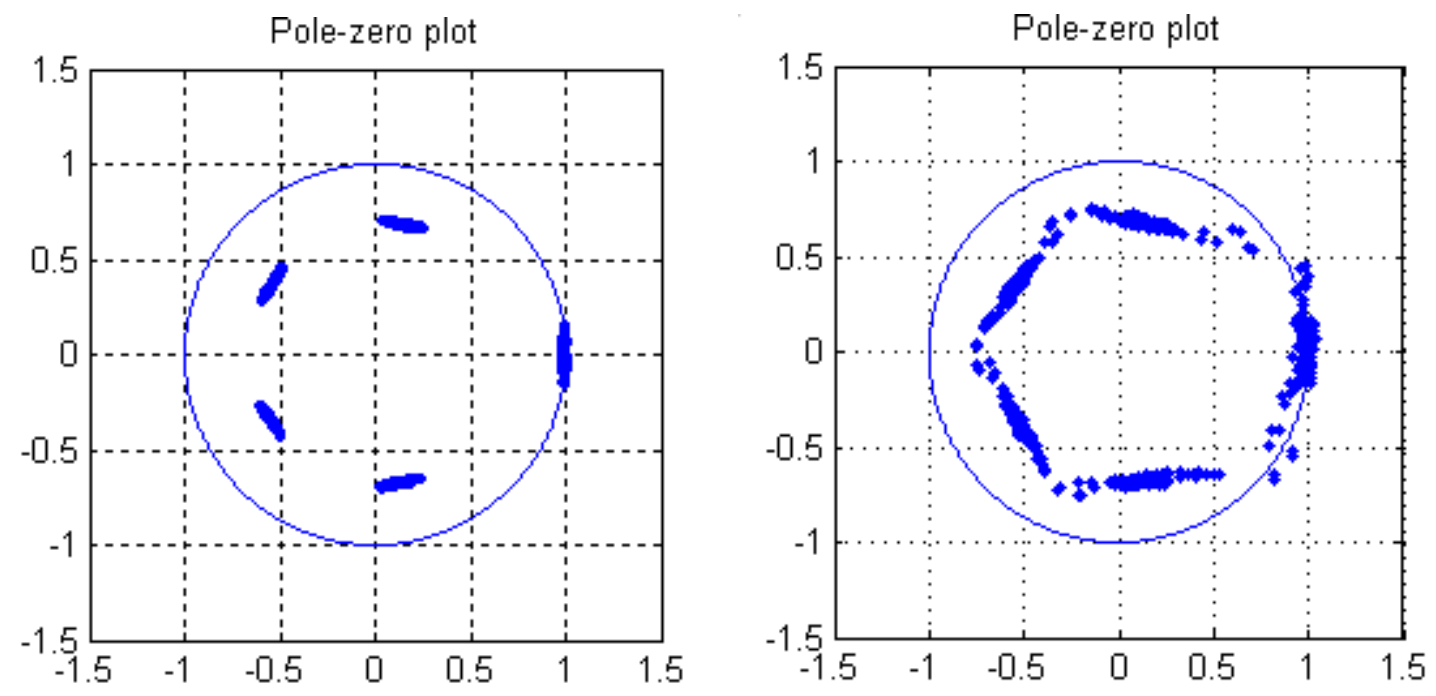

Fig. 4.4 Pole-zero plot of a fifth order filter for low noise and high noise case

\subsection{Limiter Discriminator as a Linear Predictor}

The discriminator operation is equivalent to taking the time derivative of the phase, therefore, the discriminator is actually a coarse frequency estimator. If $\alpha[n] \exp (j \phi[n])$ is the input to the limiter, where $\alpha[n]$ and $\phi[n]$ are the amplitude and phase respectively, then the output of the limiter would be $\exp (j \phi[n])$. The discriminator simply finds the difference in phase between consecutive samples by performing conjugate multiplication. The output of the discriminator can be written as

$$
z[n]=e^{j \phi[n+1]} e^{-j \phi[n]}=e^{j(\phi[n+1]-\phi[n])}
$$

The linear prediction equation for a first order filter can be written as

$$
x[n-1] a[1]=-x[n]
$$

or 


$$
a[1]=-\frac{x[n]}{x[n-1]}
$$

and the prediction polynomial is

$$
G(z)=1-\left(\frac{x[n]}{x[n-1]}\right) z^{-1}
$$

The frequency estimate is found by simply taking the angle of the filter coefficient $a[1]$. It is seen that the filter coefficient $a[1]$ is equivalent to the output of the discriminator. Therefore the discriminator can be viewed as a first order linear predictor. It seems intuitively reasonable to explore the performance of higher order predictors.

\subsection{Simulation Results}

Model-based demodulation techniques allow non-coherent demodulation. Therefore they must be compared with traditional non-coherent demodulation schemes. We have compared the performance of the model-based demodulation techniques with the limiter discriminator, as it is one of the most popular non-coherent demodulation schemes. The signal considered is Gaussian filtered MSK (GMSK) with 3-dB bandwidth bit duration product $\mathrm{BT}=0.5$.

We have used complex baseband models for our simulations. The sampling frequency $f_{s}=20$ and the symbol period is normalized to one. The sampling rate is unusually high because the tracking algorithms did not work satisfactorily for lower sampling rates. Although the model-based techniques based on the Principal Component Method did not degrade much at lower sampling rates we used the same sampling rate to make a fair comparison. The performance of the model-based demodulator in an AWGN channel using the Principal Component Method (PCM) is shown in fig. 4.5-4.9. Unless otherwise stated the signal to noise ratio $(\mathrm{Eb} / \mathrm{No})$ is $20 \mathrm{~dB}$. 
The performance of the MBD in presence of co-channel interference is shown in fig. 4.5. The performance of the limiter discriminator (LD) is also shown for reference. It is seen that MBD and LD have the same BER performance and the performance of the MBD is independent of the filter length $L$ or the number of data samples $N$. Unlike the correlation and MVU receivers the BER performance of the MBD and LD is independent of the phase difference between the two signals. The simulation results for MBD for various values of the phase difference are shown in fig. 4.6.

Next the performance of the MBD is evaluated for the case when the filter coefficients are tracked using the LMS algorithm rather than being calculated for each new symbol. The step size was chosen to be 0.005 and it was found that the prediction error was sufficiently reduced for 10 iterations of the LMS algorithm. Further iterations did not give any improvement in BER performance. The results are shown in fig. 4.7. It is seen that there is significant degradation in performance if the filter order is greater than 3 . This is because for longer filter lengths a smaller step size is required to ensure convergence and this requires a higher number of iterations.

If there is a single sinusoidal component in the signal then the angle of the first coefficient can be used as the frequency estimate [Bos92.2]. In fact, if the signal is sufficiently oversampled, all the filter coefficients have similar values and the frequency of the sinusoidal component can then be determined by finding the angle of any one of the coefficients. Simulation results for a $3^{\text {rd }}$ order filter are shown in fig. 4.8. It is seen that any of the three filter coefficients can be used without any degradation in performance. The performance of all the above schemes is compared in fig. 4.9. 


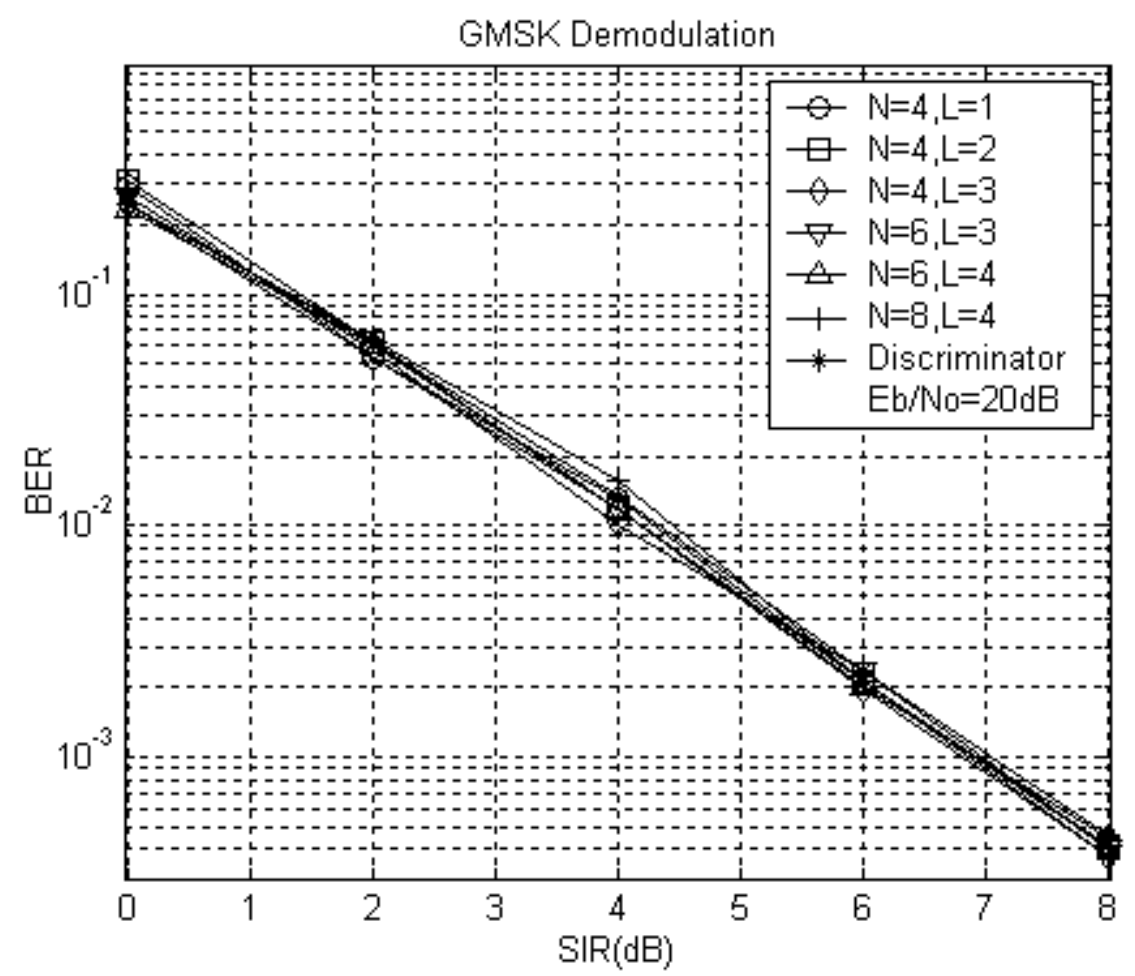

Fig. 4.5 Performance of LD and model-based demodulator for various filter lengths

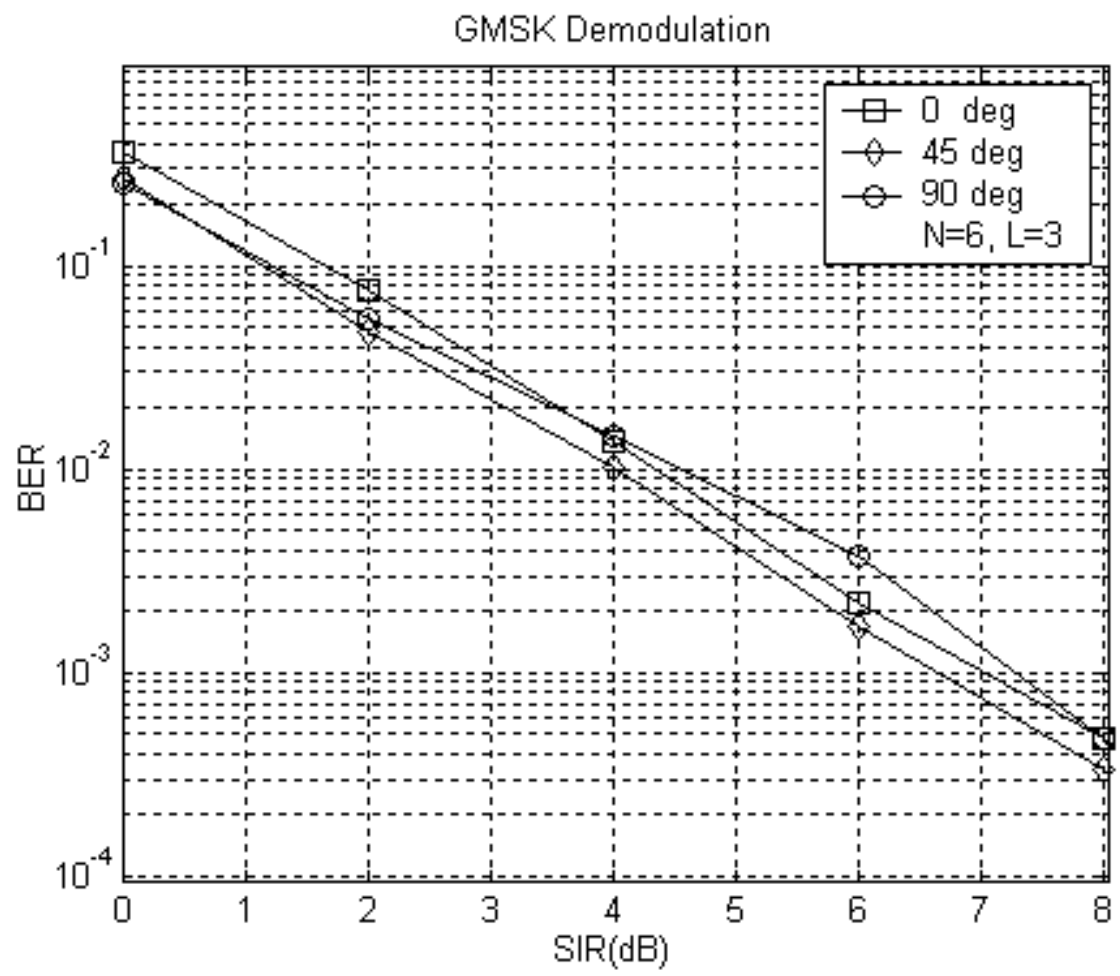

Fig. 4.6 Performance of model-based demodulator for various values of the phase difference between the signal of interest and the interferer 


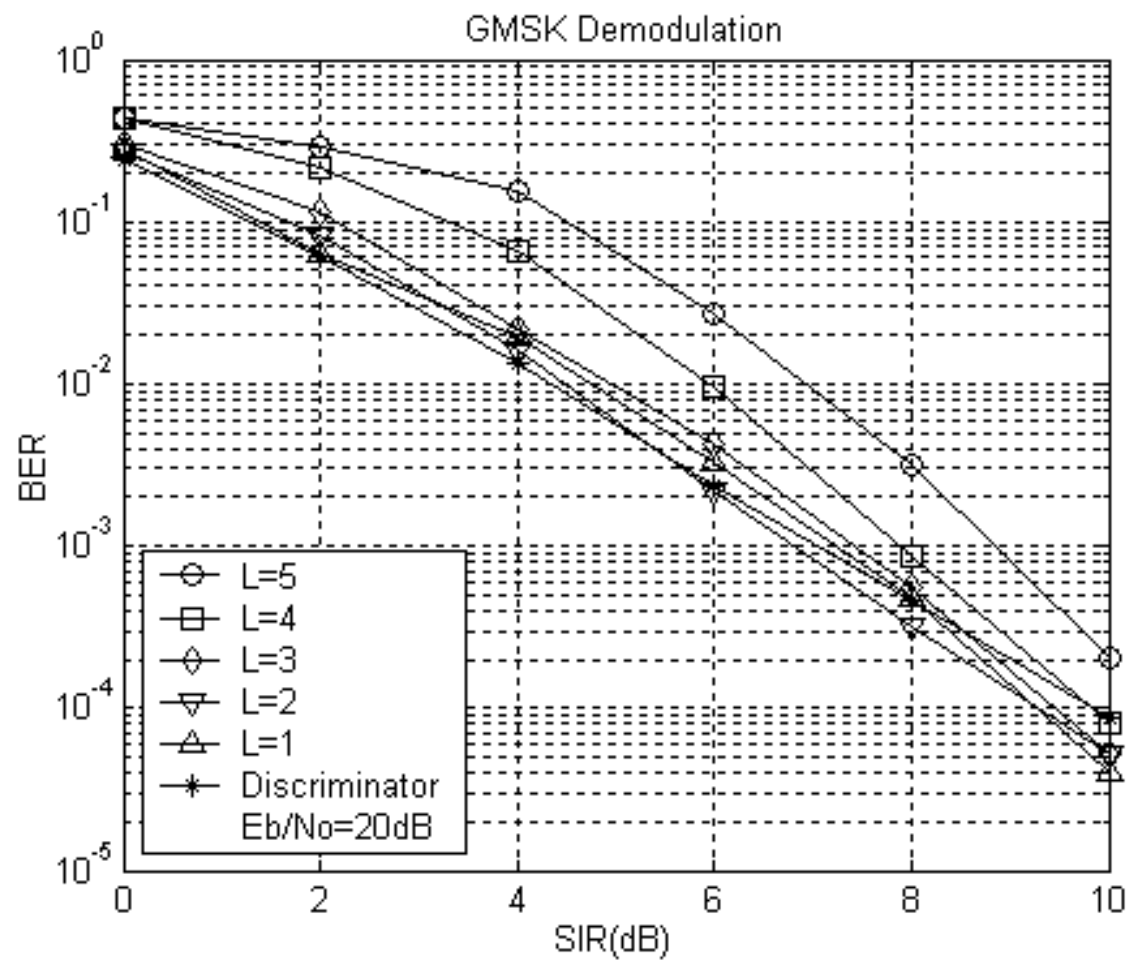

Fig. 4.7 Performance of model-based demodulator with LMS update

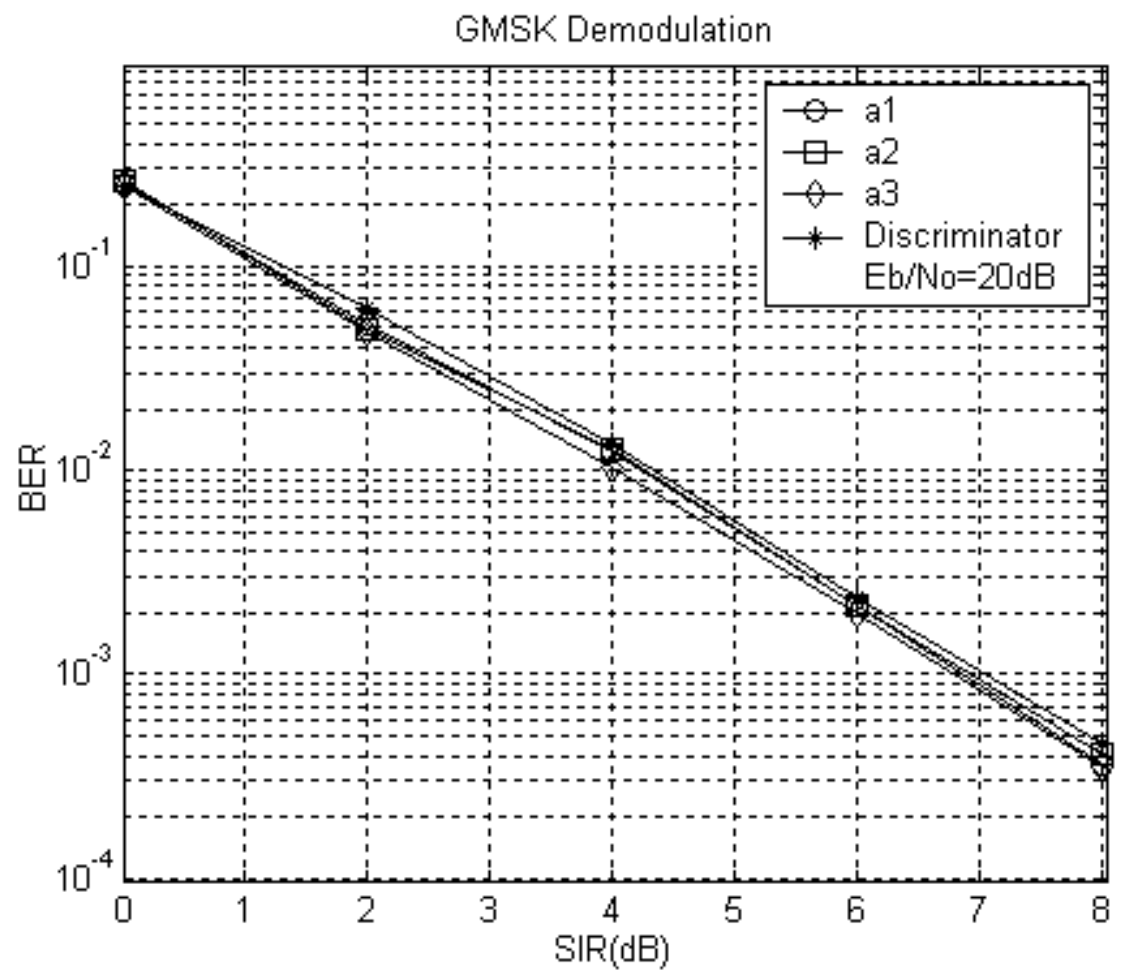

Fig. 4.8 Performance of model-based demodulator using angle of filter coefficients 


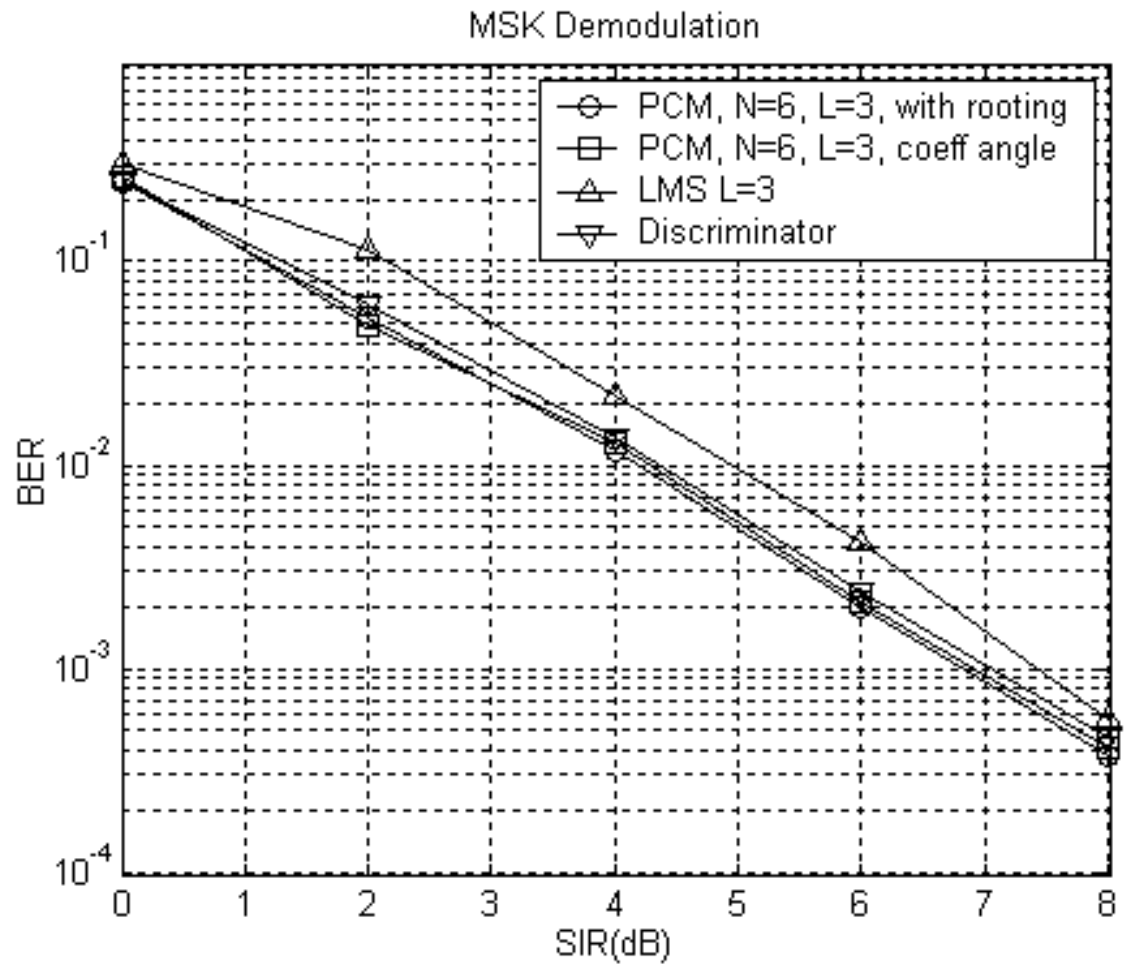

Fig. 4.9 Comparison of various model-based demodulation schemes

Since the MBD is a high-resolution frequency estimator it seems reasonable to evaluate its performance when the carrier frequencies of the two signals are not the same. As in the case of the correlation receiver the performance of the MBD degrades as the difference in carrier frequency increases. Similar results are found for the limiter discriminator. The MBD provides some improvement over the LD when the frequency difference is twice the frequency deviation $(1 / 4 \mathrm{~T})$. This is the case when one of the tones overlaps and is explained in detail in chapter 2. 


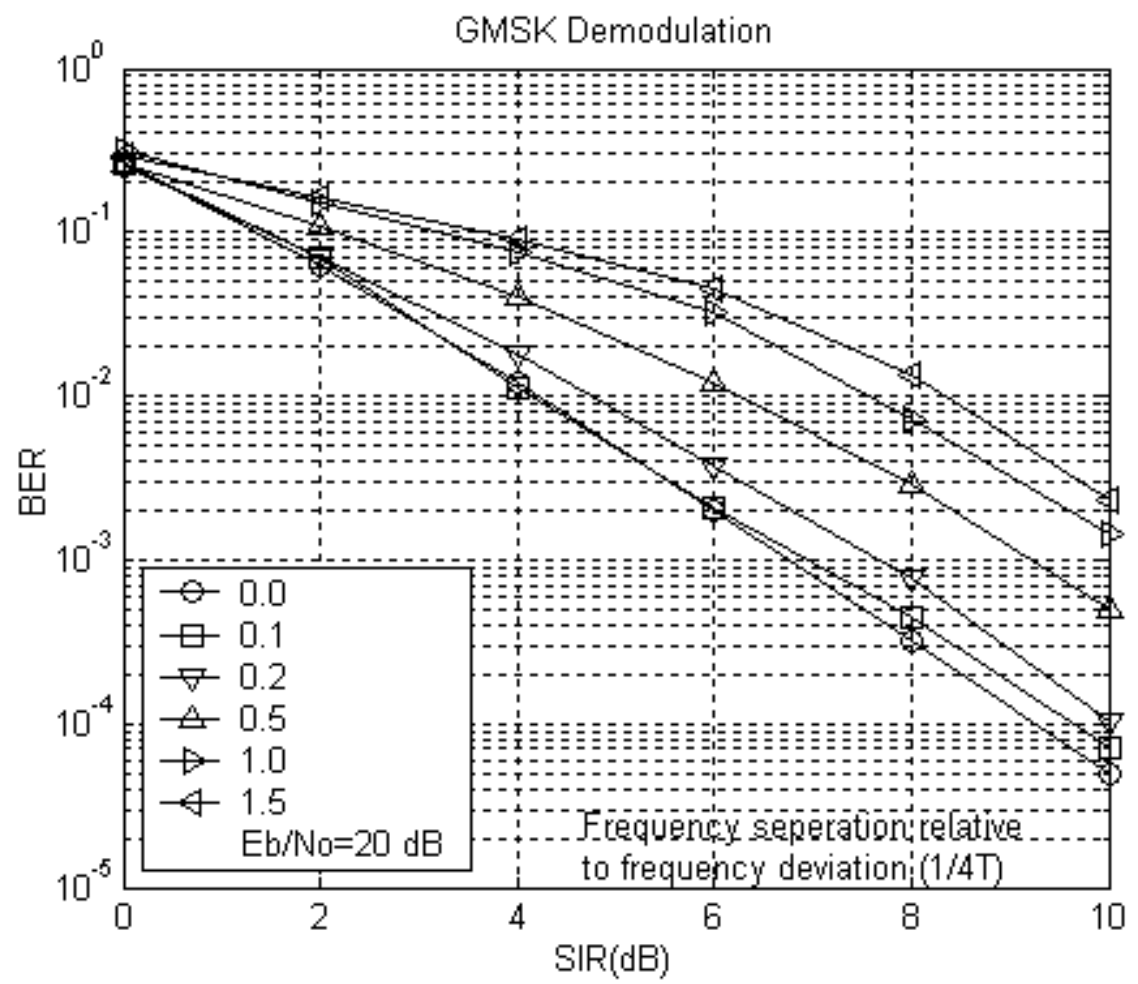

Fig. 4.10 Performance of limiter discriminator for various carrier frequency separations

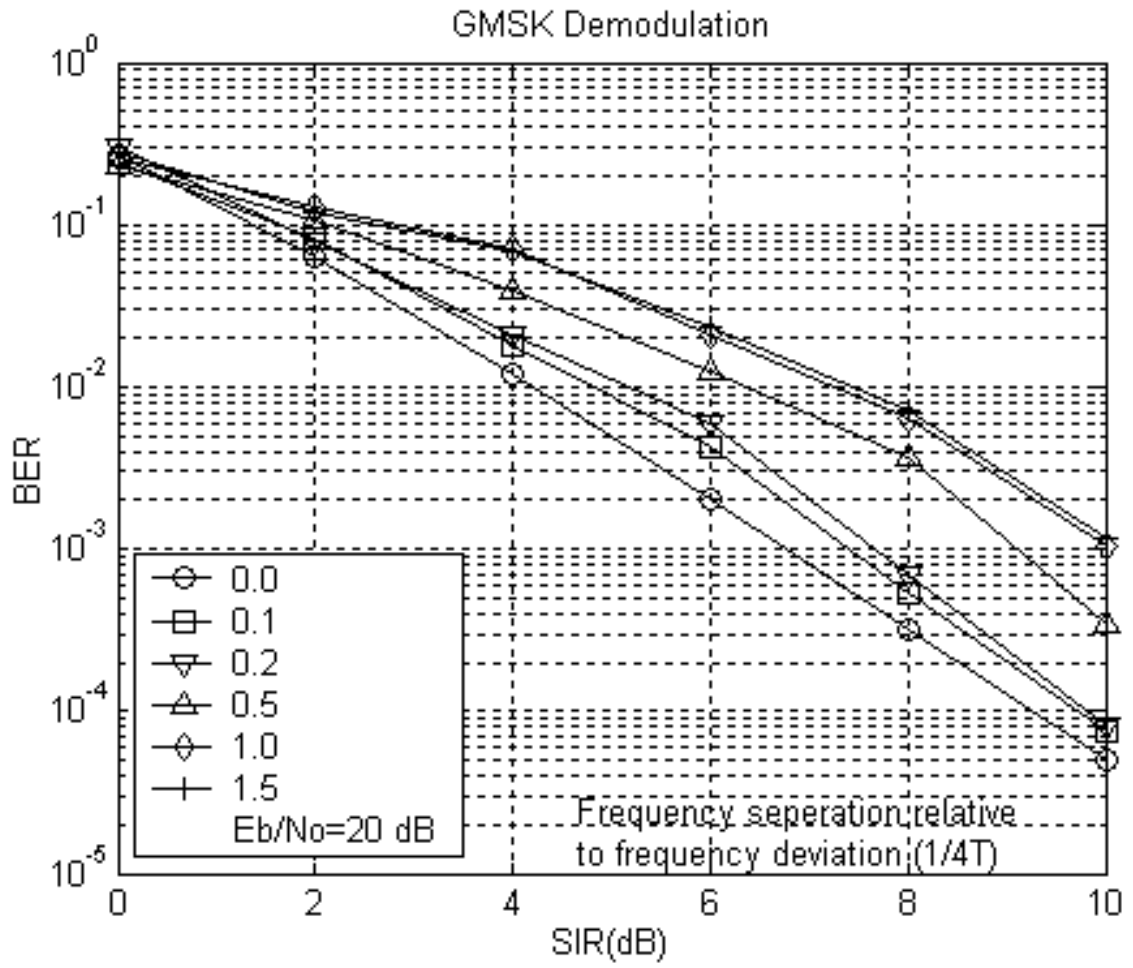

Fig. 4.11 Performance of model-based demodulator for various carrier frequency separations 


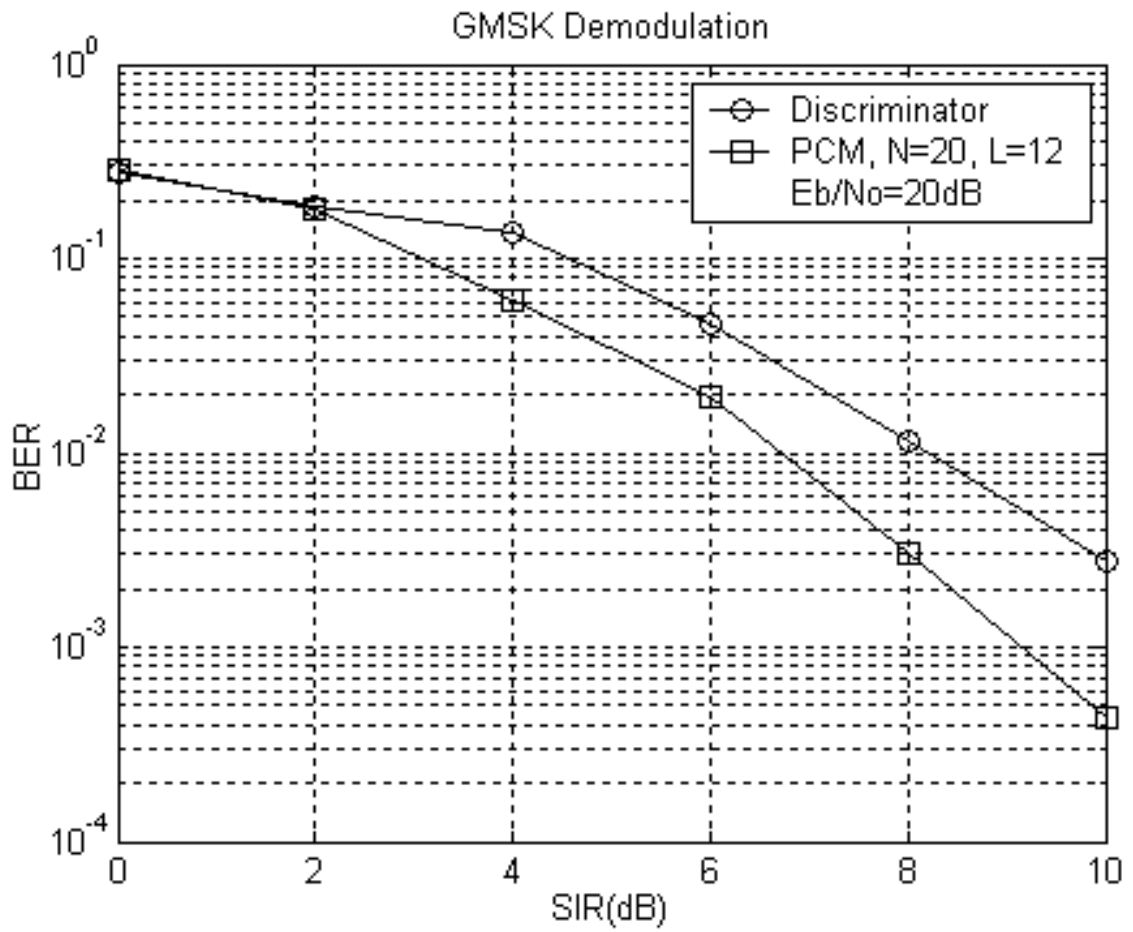

Fig. 4.12 Comparison of limiter discriminator and model based demodulator for a

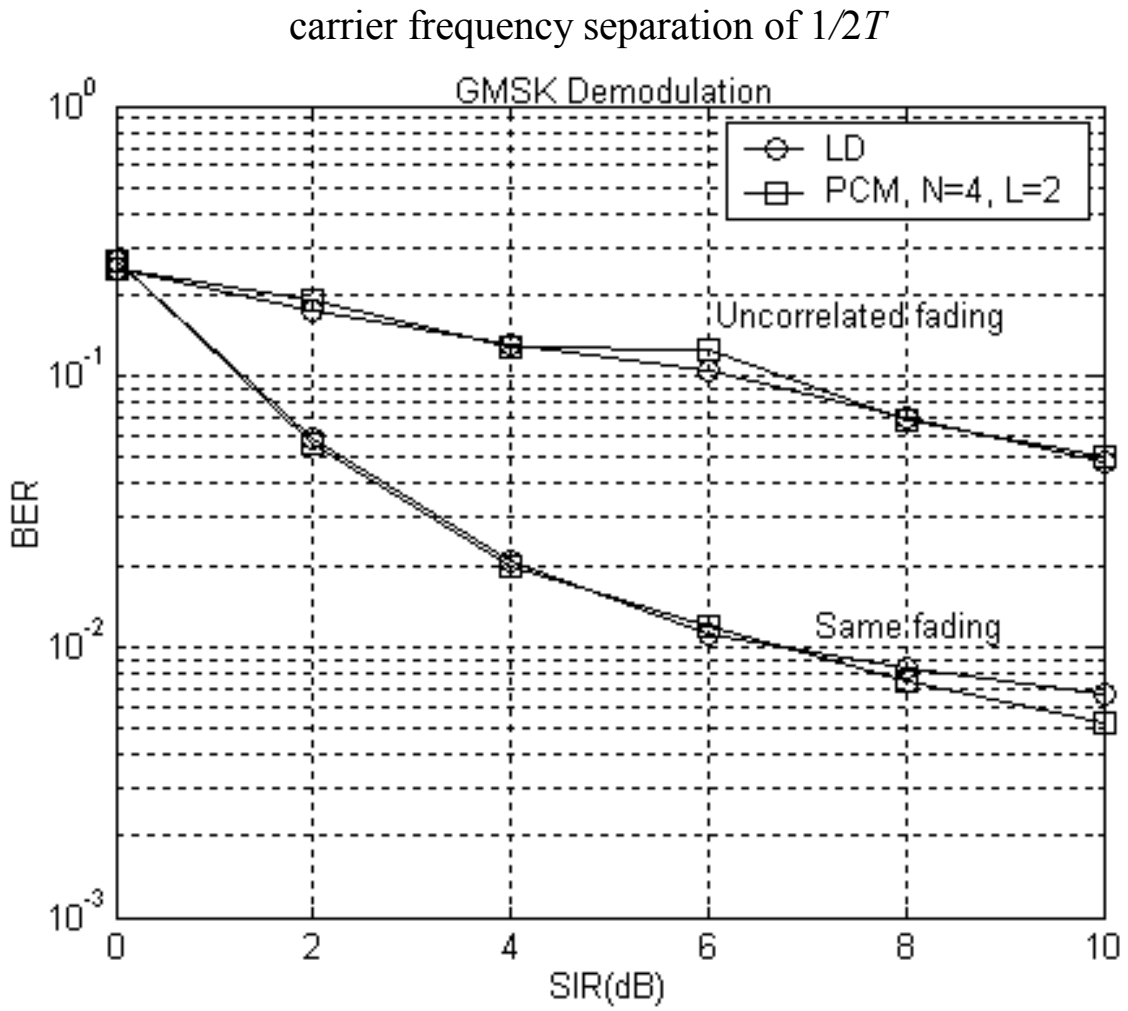

Fig. 4.13 Comparison of model-based demodulator and limiter discriminator in Rayleigh fading environment $\left(F_{d} T=0.002, E_{b} / N_{o}=20 d B\right)$ 
Finally the performance of the MBD is evaluated in a Rayleigh fading environment. We used the Clarke's and Gan's fading envelope described in [Rap96]. The fading is slow and frequency non-selective. It is seen that the performance of the MBD is similar to that of the LD. We have considered two extreme cases

1. The two signals undergo uncorrelated fading

2. The two signals undergo the same fading

It is seen that the performance is much worse for the uncorrelated fading case. This is because when the signal of interest is in a deep fade the interferer might be much stronger and this would result in a sequence of errors. However in the second case both the signals undergo the same fading and the probability of error is dependant on the relative strength of the two signals. In a practical environment the two signals would undergo correlated fading and the performance would be in between the two extremes.

\subsection{Summary}

The problem of instantaneous frequency estimation is presented and an overview of the different frequency estimation techniques is given with reference to their frequency resolution. The classical techniques based on the Fourier transform can be easily implemented using the FFT algorithm however they provide very limited frequency resolution. The model-based techniques based on rational transfer function models and subspace methods provide much higher frequency resolution. Rational transfer function models are more appropriate for modeling continuous parts of the spectrum whereas harmonic models are better suited for the discrete components in the spectrum.

The autoregressive model is selected based on the signal properties and the requirement for high frequency resolution. Different techniques for estimating the AR parameters are then discussed. The Yule-Walker equations are presented as a maximum likelihood solution for the AR parameters. The Yule-Walker method assumes that the autocorrelation function is known or is estimated from the data. The least squares approach to determining the AR parameters is then presented. This approach is much more attractive as it is directly applicable to the data and does not require the knowledge 
of the autocorrelation function. The least squares approach requires the calculation of the Moore-penrose pseudoinverse and this can be done efficiently using Singular Value Decomposition. AR parameters can be tracked using Gradient Descent Algorithms e.g. LMS and this is computationally more efficient than finding them independently for each data set.

Once the parameters have been estimated the spectral estimate can be obtained by substituting the parameters in the model. The frequency of the signal can be estimated either from the peak of the PSD or from the pole positions in the complex plain. A simple method for determining the frequency of a sinusoid from a single filter coefficient is presented. It is shown that the discriminator is basically an AR(1) frequency estimator and this motivates us to analyze the performance of higher order estimators.

Simulation results have shown that the there is no significant advantage in using a MBD over a conventional limiter discriminator in the various environments considered. The MBD provides some performance gains when the difference in carrier frequencies approaches $1 / 2 \mathrm{~T}$. 


\section{Chapter V Conclusions and Future Work}

\subsection{Conclusions}

In this work we have treated the problem of demodulation as one of parameter estimation. This is essentially a three-step procedure that involves selection of a model based upon a priori information about the data, estimation of model parameters and determining the information content of the signal from the model parameters. In the case of frequency modulated signals the last step consists of determining the instantaneous frequency from the model-parameters.

The performance of any model-based technique depends upon how accurately the model represents the data. We have considered two scenarios. In the first case we have assumed that the data model is known and that it is linear. Although this is a very simplistic assumption, it allows us to use linear estimation techniques and sets a lower bound on the performance. In the second case we have assumed that the data cannot be represented by a linear model and have used autoregressive modeling techniques.

We have shown that with proper phase difference two MSK signals are orthogonal to each other. We presented the mathematical model for this case and quantified the amount of interference when they are not orthogonal. It was noted that OMSK is actually a special case of multi-amplitude minimum shift keying (MAMSK). MAMSK is a spectrally efficient modulation scheme however it does not have a constant envelope. If a non-constant envelope modulation scheme is to be used QPSK would be preferred over MAMSK as QPSK with RRC pulse shaping has a reasonably good spectral efficiency and its error performance is superior to MAMSK. Since the second MSK signal is a cochannel interferer it is not guaranteed that it will have the required phase difference. Therefore we evaluated the performance of the demodulators over all the possible phase differences. It was observed that the performance of the correlation receiver degrades 
rapidly as the phase deviation from orthogonality increases. It was also observed that the performance of the correlation receiver degrades as the difference in carrier frequencies of the co-channel signals increases.

We next showed that the MSK signal can be represented in linear model form, hence a minimum variance unbiased (MVU) estimator exists that satisfies the Cramer-Rao lower bound (CRLB) and the solution to the MVU problem results in a typical correlation receiver. However if the basis functions are not orthogonal the exact solution needs to be calculated. The exact solution takes care of the correlation between the basis functions. These concepts are then extended to wideband signals and it is shown that the decorrelator based multiuser detector for CDMA type signals is also the MVU estimator. It seemed intuitive to use the MVU for co-channel interference rejection (in fact multiuser detection) as the two MSK signals can be considered to have non-orthogonal basis functions. Simulations results showed that the MVU does give reasonable gains in performance over the correlation receiver if the phase deviation from orthogonality is less than 30 deg. However it fails if the phase deviation is very high. Therefore the MVU will only be advantageous in an environment where we have some control over the phase difference between the two signals.

The correlation receiver and MVU are coherent demodulation schemes that require perfect carrier phase synchronization. However this is sometimes not available in practical systems. Model-based frequency estimation techniques allow non-coherent reception and they do not require the knowledge of exact data model.

We compared the performance of the model-based techniques to that of the limiter discriminator, as it is one of the most popular demodulation schemes. It is seen that the performance of the various model-based techniques is lower bounded by the limiter discriminator. Furthermore the model-based techniques do not give any significant advantage over the limiter discriminator when the carrier frequencies of the two signals are different. This is because these techniques, even with their high frequency resolution, fail to separate the two co-channel signals in the frequency domain. Two interesting 
results from the perspective of model-based demodulation were that the modelparameters need not be calculated for each new data set and can be updated using a Gradient Descent Algorithm like LMS. Secondly the frequency estimate can be found by the angle of a single filter coefficient rather than rooting the filter polynomial or finding the peak of the PSD. These two steps reduce the computational complexity of modelbased techniques without compromising the BER performance.

\subsection{Future Work}

Since the MVU estimator gives significant gains when the phase deviation from orthogonality is small and fails at higher phase deviations it might be useful to investigate the performance of the MVU in combination with other demodulation schemes that give reasonable performance at higher phase deviations. The demodulator would then have a switching mechanism that would select either of the demodulation schemes depending upon the relative phase of the two signals.

Any continuous phase modulation (CPM) can be represented by a linear model using Laurent Series approximation [Lau86]. Therefore it might also be useful to investigate the performance of the MVU for other continuous phase modulated signals e.g. MSK with different pulse shapes. We have focused on the MVU, as it is the optimum solution from a statistical point of view (minimum variance). However it might not give the best bit error rate performance. Therefore other multiuser detection techniques should also be explored. 


\section{References}

[Amr01] A. El-Hoiydi, "Interference Between Bluetooth Networks - Upper Bound on Packet Error Rate”, IEEE Communication Letters, vol. 5, issue 6, June 2001.

[Bos92.1] Boualem Boashash, "Estimating and Interpreting the Instantaneous Frequency of a Signal - Part 1: Fundamentals", Proceedings of the IEEE, vol. 80, No. 4, April 1992.

[Bos92.2] Boualem Boashash, "Estimating and Interpreting the Instantaneous Frequency of a Signal - Part 2: Algorithms and Applications", Proceedings of the IEEE, vol. 80, No. 4, April 1992.

[Box 70] Box, G.E.P. and G.M. Jenkins, Time Series Analysis: Forecasting and Control, Holden Day, San francisco, 1970.

[Eld02] Y. C. Eldar, "On Geometric Properties of the Decorrelator", IEEE

Communications Letters, vol. 6, no. 1, January 2002.

[Fai01] M. Fainberg, D. Goodman, "Analysis of the Interference between IEEE $802.11 \mathrm{~b}$ and Bluetooth Systems", Vehicular Technology Conference, 54th IEEE VTS, vol. 2, pp. $967-971,2001$.

[Gol01] N. Golmie, F. Mouveaux, "Interference in the $2.4 \mathrm{GHz}$ ISM band: Impact on the Bluetooth Access Control Performance", IEEE International Conference on Communications, vol.8, pp. 2540 -2545, 2001.

[Jun96] P. Jung and P. D. Alexander, "A Unified Approach to Multiuser Detectors for CDMA and their Geometrical Interpretations", IEEE Journal on Selected Areas in Communiations, vol. 14, no. 8, October 1996.

[Kay81] Steven M. Kay and S. Lawrence Marple Jr.,"Spectrum Analysis: A Modern Perspective", Proceedings of the IEEE, vol. 69, pp. 1380-1419, November 1981.

[Kay88] Steven M. Kay, Modern Spectral Estimation: Theory and Application, Prentice Hall, Englewood Cliffs, New Jersey, 1988.

[Kay93] Steven M. Kay, Statistical Signal Processing: Estimation Theory, Prentice Hall, Upper Saddle River, New Jersey, 1993.

[Las97] J. D. Laster and J. H. Reed, "Interference Rejection in Digital Wireless Communications", IEEE Signal Processing Magazine, vol. 14, issue 3, pp. 37-62, May 1997.

[Lau86] Pierre A. Laurent, "Exact and Approximate Construction of Digital Phase Modulations By Superposition of Amplitude Modulated Pulses (AMP)," IEEE Transactions on Communications, vol. COM-34, no. 2, pp. 150-160, February 1986. 
[Mak75] John Makhoul, "Linear Prediction: A Tutorial Review", Proceedings of the IEEE, vol. 63, pp. 561-580, April 1975.

[Man00] Dimitris G. Manolakis, Vinay K. Ingle and Stephen M. Kogon, Statistical and Adaptive Signal Processing, McGraw-Hill, 2000.

[Mph96] P. A Murphy and G. E. Ford, "Co-channel Demodulation for Continuous-Phase Modulated Signals", $29^{\text {th }}$ Asilomar Conference on Signals Systems and Computers, vol. 1, pp. 330-334, Nov 1995.

[Mur81] K. Murota and K. Hirade, "GSMK Modulation for Digital Mobile Telephony", IEEE Transactions on Communications, vol. COM 29, no. 7, pp. 1044-1050, July 1981.

[Pas79] S. Pasupathy, "Minimum Shift Keying: A Spectrally Efficient Modulation”, IEEE Communications Magazine, vol. 17, no. 4, pp. 14-22, July 1979.

[Por01] H. Vincent Poor, "Active Interference Suppression in CDMA overlay systems", IEEE Journal on Selected Areas in Communications, vol. 19, no. 1, January 2001.

[Por87] Porat, B. and B. Friedlander, "The Exact Cramer-Rao Bound for Gaussian Autoregressive Processes", IEEE Trans. Aerosp. Electron. Syst., Vol. 23, pp. 537-541, July 1987.

[Pro01] John G. Proakis, Digital Communications, Fourth Edition, McGraw-Hill 2001.

[QLi00] Q. Li, and C N. Georghiades, "On a Geometric View of Multiuser Detection for Synchronous DS/CDMA Channels" IEEE Transactions on Information Theory, vol. 46, no. 7, November 2000.

[Ran95] P. A. Ranta, A. Hottinen and Z. C. Honkasalo, "Co-Channel Interference Cancelling Receiver for TDMA Mobile Systems", IEEE International Conference on Communications, vol. 1, pp. 17-21, 1995.

[Rap96] T. S. Rappaport, Wireless Communications: Principles and Practice, Prentice Hall, Upper Saddle River, New Jersey, 1996.

[Ras96] L. K. Rasmussen, T. J. Lim and P. D. Alexander, "A New Geometrical Interpretation of the Decorrelator in Multiuser CDMA", IEEE Global Telecommunications Conference, 1996.

[Roy89] Richard Roy and Thomas Kailath, "ESPRIT - Estimation of Signal Parameters Via Rotational Invariance Techniques", IEEE Transactions on Acoustic Speech and Signal Processing, vol. 37, no. 7, July 1989. 
[Saf00] S. Bayram, J. Hicks, R. J. Boyle, J. H. Reed, "Overloaded Array Processing in Wireless Airborne Communication Systems", 21st Century Military Communications Conference Proceedings, vol. 1.1, pp. 24 -29, 2000.

[Sk188] Bernard Sklar, Digital Communications: Fundamental and Applications, Prentice Hall, Upper Saddle River, New Jersey, 1988

[Sun86] C. E. Sundberg, "Continuous Phase Modulation: A Class of Jointly Power and Bandwidth Efficient Digital Modulation Schemes with Constant Amplitude", IEEE Communications Magazine, vol. 24, no. 4, pp. 25-38, April 1986.

[The92] Charles W. Therrien, Discrete Random Signals and Statistical Signal Processing, Prentice Hall, Englewood Cliffs, New Jersey, 1992.

[Ver98] S. Verdu, Multiuser Detection, Cambridge University Press, Cambridge, 1998. http://www.cup.cam.ac.uk

[Web78] W. J. Weber III, P. H. Stanton and J. T. Sumida, “A Bandwidth Compressive Modulation System Using Multi-Amplitude Minimum Shift Keying”, IEEE Transactions on Communications, vol. com-26, no. 5, May 1978.

[We196] Mathew L. Welborn, "Co-Channel Interference Rejection Using a Model-Based Demodulator", MS Thesis, Virginia Polytechnic Institute and State University, January 1995. 
Appendix A

\section{Appendix A}

The power spectral density (PSD) of an autoregressive (AR) process can be written in terms of the estimated AR parameters $\hat{a}[m]$ as [Kay93]

$$
\hat{P}_{x x}(f)=\frac{\hat{\sigma}_{u}^{2}}{\left|1+\sum_{m=1}^{p} \hat{a}[m] \mathrm{e}^{-j 2 \pi f m}\right|^{2}}
$$

for $p=1$ this becomes

$$
\hat{P}_{x x}(f)=\frac{\hat{\sigma}_{u}{ }^{2}}{\left|1+\hat{a}[1] \mathrm{e}^{-j 2 \pi f}\right|^{2}}
$$

The Cramer-Rao lower bound for AR parameters can be written as [Kay93]

$$
\operatorname{var}(\hat{a}[k]) \geq \frac{\sigma_{u}^{2}}{N}\left[R_{x x}^{-1}\right]_{k k} \quad k=1,2 \ldots p
$$

For a first order AR process

$$
r_{x x}[0]=\frac{\sigma_{u}{ }^{2}}{1-a^{2}[1]}
$$

therefore

$\operatorname{var}(\hat{a}[1]) \geq \frac{1-a^{2}[1]}{N}$

The CRLB for the PSD of an AR process can then be found using transformation of parameters

$$
\operatorname{var}\left(\hat{P}_{x x}(f)\right) \geq \frac{\left(\frac{\partial \hat{P}_{x x}(f)}{\partial a[1]}\right)^{2}}{-E\left[\frac{\partial^{2} \ln p(x ; \theta)}{\partial \theta^{2}}\right]}
$$

or 
Appendix A

$\operatorname{var}\left(\hat{P}_{x x}(f)\right) \geq\left(\frac{\partial \hat{P}_{x x}(f)}{\partial a[1]}\right)^{2} \operatorname{var}(\hat{a}[1])_{C R L B}$

Here

$$
\begin{aligned}
& \frac{\partial}{\partial a[1]} \hat{P}_{x x}(f) \\
& =\frac{\partial}{\partial a[1]} \frac{\sigma_{u}^{2}}{\left|1+a[1] e^{-j 2 \pi f}\right|^{2}} \\
& =\frac{\partial}{\partial a[1]} \frac{\sigma_{u}^{2}}{\left(1+a[1] e^{j 2 \pi f}\right)\left(1+a[1] e^{-j 2 \pi f}\right)} \\
& =\frac{\partial}{\partial a[1]} \frac{\sigma_{u}^{2}}{1+a[1] e^{j 2 \pi f}+a[1] e^{-j 2 \pi f}+a^{2}[1]} \\
& =\frac{\partial}{\partial a[1]} \frac{\sigma_{u}^{2}}{1+2 a[1] \cos (2 \pi f)+a^{2}[1]} \\
& =-\sigma_{u}^{2} \frac{(2 \cos (2 \pi f)+2 a[1])}{\left(1+2 a[1] \cos (2 \pi f)+a^{2}[1]\right)^{2}}
\end{aligned}
$$

therefore

$$
\left(\frac{d}{d a[1]} \hat{P}_{x x}(f)\right)^{2}=\frac{4 \sigma_{u}{ }^{4}(a[1]+\cos (2 \pi f))^{2}}{\left(1+a^{2}[1]+2 a[1] \cos (2 \pi f)\right)^{4}}
$$

and

$$
\operatorname{var}\left(\hat{P}_{x x}(f)\right) \geq \frac{4 \sigma_{u}^{4}\left(1-a^{2}[1]\right)(a[1]+\cos (2 \pi f))^{2}}{N\left(1+a^{2}[1]+2 a[1] \cos (2 \pi f)\right)^{4}}
$$




\section{Vita}

Yasir Ahmed was born on March 26, 1977 in Hyderabad, Pakistan. He graduated from the National University of Sciences and Technology, Karachi, Pakistan in January 1999 with a Bachelor's Degree in Electrical Engineering and then worked as a Design Engineer at Data Communication and Controls Pvt. Ltd. from April 1999 to June 2000. He joined the graduate program at Virginia Tech in August 2000 and has been working as a Graduate Research Assistant at the Mobile and Portable Radio Research Group since January 2001. His research interests include interference cancellation, multiuser detection, spectral estimation and antenna arrays. 